Cells in Motion Viewpoints

\title{
Preface
}

\section{Emerging aspects of leukocyte migration}

\author{
Reinhold Förster $^{1}$ and Silvano Sozzani ${ }^{2,3}$ \\ ${ }^{1}$ Institute of Immunology, Hannover Medical School, Hannover, Germany \\ 2 Department Molecular and Translational Medicine, University of Brescia, Brescia, Italy \\ ${ }^{3}$ Humanitas Clinical and Research Center, Rozzano, Italy
}

DOI: 10.1002/eji.201343670

\section{Introduction}

The motility of its cellular components is a key feature of the immune system since it allows the selective recruitment of cells to defined places and niches not only to combat invading pathogens but also to pass through tightly controlled programs of immune cell development and maturation. In nature, two profoundly different processes contribute to immune cell motility: passive transport in blood and lymph fluid between different lymphoid and nonlymphoid organs, and active migratory processes that control entry and exit to and from lymphoid organs as well as their precise positioning therein. Furthermore, active cell migration is a key process that allows the entry and exit of effector cells to places of infection and inflammation. It is generally accepted that the passive, bloodmediated transport of immune cells to lymphoid and nonlymphoid organs occurs as a random, noncontrolled process. In contrast, a plethora of studies in the past two decades have revealed many of the cellular and molecular cues that control immune cell migration to and within defined compartments. While passive transport allows rapid cellular distribution and dissemination throughout the body, active migratory processes are of paramount importance to tightly control the immune system. Passive transport and active migration provide the basis of all immune responses not only in vertebrates but also in all multicellular organisms that harbor motile immune cells with defined effector functions. This Viewpoints series addresses several emerging aspects of leukocyte migration.

\section{Key players in immune cell migration}

Leukocyte migration from the blood compartment into tissues is a process that was first reported almost 200 years ago and molecularly defined as a multistep process in the early 1990s of the past century [1]. Additional studies have subsequently defined the molecules and the sequence of the events involved in leukocyteendothelial cell interaction [2-5]. Leukocyte extravasation is now envisioned as a process that undergoes the following sequential steps: tethering, rolling, activation, adhesion, crawling, and transmigration, with each step relying on the function of a defined set of molecules. The expression of $L$-selectin by leukocytes and $P$ - and $E$-selectins by activated endothelial cells are mostly responsible for tethering and rolling of leukocytes on the luminal endothelial blood surface. Selectins interact with cognate glycosylated ligands expressed by the interacting cells. On the other hand, leukocyte activation and arrest on endothelium is rapidly induced by the engagement of leukocyte chemotactic receptors by chemotactic factors immobilized by glycosaminoglycans or heparan sulphates. The "inside-out" signaling generated by activated chemotactic receptors is crucial for the transition of integrins from the low to the high affinity conformation and for the arrest of leukocytes on activated endothelial cells [6]. A more recent contribution to this scenario is emerging from the understanding of the role of the so-called family of the atypical chemokine receptors (ACKRs).
ACKRs are seven-transmembrane spanning proteins that bind chemotactic factors in a promiscuous manner in the absence of the classic G-protein-coupled signaling that characterizes chemotactic receptors. ACKRs, located at either the haematic or lymphatic endothelial barriers, are now believed to shape the chemotactic gradient through the degradation of secreted chemokines or the presentation/concentration of chemokines on the surface of activated endothelial cells. In the Viewpoint series in this issue, Del Prete et al. summarize recent insights into an ACKR named CCRL2 [7].

Chemotactic factors include pathogenassociated molecular patterns such as formylated peptides, complement proteins (e.g. C5a and C3a), lipids (e.g. PAF and LTB4), and chemokines [8]. The Viewpoint by Yang et al. summarizes the role of alarmins, a new heterogeneous family of chemotactic agonists [9]. Alarmins include different types of molecules, such as antimicrobial peptides, nuclear-binding proteins, heat shock proteins, nucleotides/metabolites, and granule proteins released by stressed cells. Alarmins induce the recruitment and activation of antigen presenting cells, as well as other immune cells, often in cooperation with other chemotactic factors through the activation of different types of receptors, many of them still uncharacterized.

\section{Impaired migration in primary immunodeficiency}

The crucial role of leukocyte recruitment in immune responses is witnessed by the 
clinical manifestations associated with genetic defects in cell adhesion, actin cytoskeleton reorganization, and chemokine receptor signaling that are reviewed in the Viewpoint by Badolato [10]. Historically, the first such evidence was obtained in patients with mutations in the gene encoding CD18, the $\beta 2$ integrin chain (LAD I; leukocyte adhesion deficiency-1) and in the gene encoding for a GDP-fucose transporter involved in the synthesis of selectin ligands (LAD II). More recently, a third genetic adhesion defect, characterized by mutations in the gene encoding for kindlin-3, a protein involved in integrin activation, has been reported and named LAD III. All these patients present neutrophilia and increased susceptibility to bacterial infections. Since LAD III patients present a general defect in integrin activation, they also experience defects in platelet activation and osteoclast functions [11]. A defect in actin polymerization known as Wiskott-Aldrich Syndrome (WAS) is caused by mutations in a protein, the WAS protein (WASP), involved in actin polymerization. Mutations in WASP are responsible for four related diseases characterized by microthrombocytopenia, eczema, and recurrent bacterial infections due to the reduction in the number of naïve $\mathrm{T}$ cells, decreased antibody response and impaired migration of innate effector cells and lymphocytes [12]. Finally, the WHIM (warts, hypogammaglobulinemia, infections, and myelokathexis) syndrome represents the first identified example of human disease characterized by a genetic defect of a chemokine receptor. In WHIM patients, the expression of truncated forms of the $C$-terminal tail of the receptor is associated with a prolonged response to CXCL12, the CXCR4 cognate ligand, which results in neutrophil bone marrow retention. Patients with WHIM syndrome have increased bacterial infections, especially of the respiratory tract; in agreement with the phenotype originally observed in mice deficient for the CXCL12 receptors, CXCR4 and CXCR7 [13,14], WHIM patients also show a higher rate of congenital heart defects.

\section{Migration and induction of tolerance}

Tolerance to self and innocuous antigens is provided by two main mechanisms, namely central tolerance in primary lymphoid organs and peripheral tolerance in secondary lymphoid organs and peripheral tissues. Chemokines were shown to be profoundly involved in both types of tolerance acting at the level of T-cell and DC recruitment [15]. T-cell central tolerance is induced in the thymus and a nonredundant role of chemokines was shown in the trafficking of progenitor cells from the cortex to the medulla [16]. Chemokines were also shown to play a crucial role in the recruitment and positioning of thymic DC subsets. In their Viewpoint, Hadeiba and Butcher discuss the role of three main subsets of DCs present in the thymus, each of them endowed with specific roles in $\mathrm{T}$ cell clonal deletion and Treg-cell induction [17]. The recruitment and correct homing of each of these populations was shown to be dependent on the expression of specific chemokine receptors, such as CCR9 for plasmacytoid DCs, XCR1 for resident DCs and CCR2 for migrating conventional DCs [18-20]. Similarly, Treg cells, which represent one of the main effector mechanisms of peripheral tolerance, home, and recirculate through the expression of specific chemokine receptors. Thymic-derived natural Treg cells leave the thymus and migrate to secondary lymphoid organs through the expression of CCR7 and CD62L. On the contrary, inducible Treg cells need to express selective homing receptors to recirculate between the lymph nodes and the extra-lymphatic tissues, such as gut or skin, in order to properly shape their function. In their Viewpoint, Pabst and Bernhardt focus on the mechanisms that control the generation, maintenance, and function of Treg cells in the digestive tract [21].

\section{New insights in cell migra- tion behavior by novel imaging techniques}

During the past decade, two-photon laser scanning microscopy (2-PM) has been applied with great success to reveal the positioning, movement, and interaction dynamics of immune cells within intact tissues. Compared with conventional epifluorescence or confocal microscopy, 2-PM provides better penetration depth in combination with little tissue damage while the spatial resolution is of poorer quality. Since 2-PM has been frequently used in intravital experimental settings, the analysis of cellular interactions taking place within living, anaesthetized animals has offered profound new insights into immune cell motility and function. In contrast to conventional fluorescence microscopy, which uses excitation wavelengths within the visible spectrum, in 2-PM a single fluorochrome molecule has to be hit not by one but, nearly simultaneously, by two infrared photons. In order to achieve excitation, these 2 photons are of approximately double the wavelength and thus half the energy [22]. This approach offers several advantages. First, due to its longer wavelength, the infrared excitation light is scattered less that yields higher penetration depth. Second, only within a very small volume within the focal point of the optical system is the probability high enough that near-simultaneous interactions of two infrared photons with the very same fluorochrome molecule actually result in its excitation. Thus, generation of "out-of-focus" light as well as fluorochrome bleaching outside of the focal plane is intrinsically eliminated, which helps to conserve the specimen and allows continuous imaging of the very same region for several hours. Tang et al. discuss, in their Viewpoint [23], recent advantages and the so far unmet needs in fluorescencebased optical imaging that are required to further dissect complex cell-cell interactions and to visualize the functional consequences for immunity and tolerance. Tang et al. [23] discuss five important challenges - imaging depth, multiplex detection, faster imaging, higher resolution, and data capture and processing - that require considerable improvement to gain substantially new insights into the processes that control cell motility and the functional consequences of these processes.

\section{Interfering with cell migration as novel therapeutic approaches}

As outlined above, the homing of immune cells to defined organs and subcompartments therein is controlled by the orchestrated expression of several classes of molecules required for cell homing. This feature not only allows the controlled access to tissues but also offers new therapeutic approaches that interfere with selective steps of the cell homing cascade. Griffith and Luster's Viewpoint gives an overview of the molecules involved in cell homing during inflammatory processes that are currently targets in clinical trials. In particular, they focus on 
two compounds: Natalizumab and Sphingolimod (FTY720) [24]. Natalizumab is a humanized mAb that blocks $\alpha 4$ integrins and thus interferes with $\alpha 4 \beta 7$ integrinmediated homing of cells to the intestine and $\alpha 4 \beta 1$ integrin-dependent homing to the brain. This drug has shown very promising results in treating patients with inflammatory bowel disease and multiple sclerosis $[25,26]$. However, treatment of patients with this antibody also creates new challenges. In some patients the neurotropic JC virus, which is present in more than $50 \%$ of the healthy adult population, cannot be controlled, leading to progressive multifocal leukoencephalopathy [27]. Sphingolimod is also being tested in clinical studies in patients with MS. However, the mode of action is rather different. It binds to four of the five sphingosine1-phosphate receptors [28] and evidence is accumulating that Sphingolimod mediates its therapeutic effects by inhibiting the egress of activated $\mathrm{T}$ cells from secondary lymphoid organs [29]. Thus, it seems that the trapping of activated cells in lymphoid organs represents the main mode of action of this substance.

\section{Conclusions}

Based on fundamental experiments of the past two decades, the mechanisms that regulate egress of immune cells from blood to lymphoid and nonlymphoid tissue are largely understood. In contrast, hardly anything is known regarding the homing of afferent lymph-derived cells to lymphoid organs. This open field requires further investigation since many of the fundamental processes of immunology, such as DC mobilization from the peripheral organs to the draining lymph nodes, rely on this trafficking route. New imaging techniques like 2-photon and light-sheet microscopy will be of profound value in gaining a better understanding of the molecular and cellular mechanisms that regulate immune cell trafficking from and to the bloodstream as well as the lymphatic system. The selective interference with immune cell homing or egress to or from defined lymphoid and nonlymphoid organs offers new therapeutic strategies to dampen chronic inflammatory processes. Several biologics, in particular those directed against different integrins and selectins, are currently being tested in clinical trials and show very promising results. Although belonging to the large and frequently therapeutically targeted family of G protein-coupled receptors, the therapeutic potential of targeting chemoattractant receptors is still largely unexploited. Clearly, further studies are needed to better delineate the capacity of this receptor class as drug targets.

Acknowledgments: The work of R.F. is supported by grants of the Deutsche Forschungsgemeinschaft DFG (SFB621A1; SFB587-B3; SFB738-B5, SFB900B1; FO334/5-1) and the European Research Council (ERC Advanced Grant 322645, Lymphatics-Homing). S.S. is supported by AIRC (Associazione Italiana per la Ricerca sul Cancro) and Ministero dell'Istruzione, dell'Università e della Ricerca (M.I.U.R.), European Project IMI JU-funded project BeTheCure, contract 115142-2.

Conflict of interest: The authors declare no financial or commercial conflict of interest.

\section{References}

1 Butcher, E. C., Cell 1991. 67: 1033-1036.

2 Springer, T. A., Annu. Rev. Physiol. 1995. 57: 827-872.

3 Ley, K. et al., Nat. Rev. Immunol. 2007. 7: 678-689.

4 Sanz, M. J. and Kubes, P., Eur. J. Immunol. 2012. 42: $278-283$.

5 Salmi, M. and Jalkanen, S., Eur. J. Immunol. 2012. 42: 284-292.

6 Girard, J. P. et al., Nat. Rev. Immunol. 2012. 12: 762-773.

7 Del Prete, A. et al., Eur. J. Immunol. 2013. 43: 1418-1422.

8 Sozzani, S., Cytokine Growth Factor Rev. 2005. 16: 581-592.

9 Yang, D. et al., Eur. J. Immunol. 2013. 43: 14121418.

10 Badolato, R., Eur. J. Immunol. 2013. 43: 14351440.

11 Etzioni, A., Curr. Opin. Immunol. 2009. 21: 481-486.

12 Thrasher, A. J. and Burns, S. O., Nat. Rev. Immunol. 2010. 10: 182-192.

13 Sierro, F. et al., Proc. Natl. Acad. Sci. USA 2007. 104: 14759-14764.
14 Zou, Y. R. et al., Nature 1998. 393 595-599.

15 Liu, X. et al., Eur. J. Immunol. 2011. 41 611-623.

16 Bunting, M. D. et al., Immunol. Cell Biol. 2011. 89: 185-196.

17 Hadeiba, H. and Butcher, E. C., Eur. J. Immunol. 2013. 43: 1425-1429.

18 Baba, T. et al., J. Immunol. 2009. 183: 3053-3063.

19 Hadeiba, H. et al., Immunity 2012. 36 438-450.

20 Lei, Y. et al., J. Exp. Med. 2011. 208: 383-394.

21 Pabst, O. and Bernhardt, G., Eur. J. Immunol. 2013. 43: 1422-1425.

22 Cahalan, M. D. et al., Nat. Rev. Immunol. 2002 2: $872-880$

23 Tang, J. et al., Eur. J. Immunol. 2013. 43: 1412 1418.

24 Griffith, J. W. and Luster, A. D., Eur. J. Immunol. 2013. 43: 1429-1435.

25 Ransohoff, R. M., N Engl. J. Med. 2007. 356 2622-2629.

26 Ghosh, N. et al., Int. Rev. Immunol. 2012. 31: 410-427.

27 Berger, J. R. and Koralnik, I. J., N. Engl. J. Med. 2005. 353: 414-416.

28 Mandala, S. et al., Science 2002. 296 346-349.

29 Cyster, J. G. and Schwab, S. R., Annu. Rev. Immunol. 2012. 30: 69-94.

Correspondence: Prof. Reinhold Forster, Institute of Immunology, Hannover Medical School, Carl-Neuberg-Str. 1, Hannover 30625 , Germany

e-mail: foerster.reinhold@mh-hannover.de

Additional correspondence: Prof. Silvano

Sozzani; Department Molecular and

Translational Medicine, University of Brescia, Italy

e-mail: sozzani@med.unibs.it

Received: 30/4/2013

Revised: 30/4/2013

Accepted: 7/5/2013

Keywords: Cell migration - Immunodeficiencies · Immunotherapy

Abbreviations: ACKR: atypical chemokine receptor . WAS: Wiskott-Aldrich syndrome WHIM: warts, hypogammaglobulinemia, infections, and myelokathexis

The complete Cells in Motion Viewpoint series is available at: http://onlinelibrary.wiley.com/doi/10. 1002/eji.v43.6/issuetoc 


\title{
Technologies to observe and understand cells in motion
}

\section{The future of immunoimaging - Deeper, bigger, more precise, and definitively more colorful}

\author{
Jianyong Tang, Nicolas van Panhuys, Wolfgang Kastenmüller and Ronald N. Germain \\ Lymphocyte Biology Section, Laboratory of Systems Biology, National Institute of Allergy \\ and Infectious Diseases, National Institutes of Health, Bethesda, MD, USA
}

DOI: 10.1002/eji.201243119

Immune cells are thoroughbreds, moving farther and faster and surveying more diverse tissue space than their nonhematopoietic brethren. Intravital 2-photon microscopy has provided insights into the movements and interactions of many immune cell types in diverse tissues, but more information is needed to link such analyses of dynamic cell behavior to function. Here, we describe additional methods whose application promises to extend our vision, allowing more complete, multiscale dissection of how immune cell positioning and movement are linked to system state, host defense, and disease.

The immune system is like a fine mechanical watch - there are a large number of parts that must work together to achieve the right result. For the watch, the goal is keeping perfect time, and for the immune system, the goal is optimally protecting the host. If the pieces are not machined and assembled properly, the watch (immune system) can run too slowly (immunodeficiency) or too fast (autoimmunity/ inflammatory disease). There is a delicate balance in the interaction of the watch parts - they must move properly, engage for just the right amount of time, then disengage, and move again. Likewise, the cells of the immune system must circulate and migrate, find the right cellular partner at the right time, engage for the proper duration, signal effectively, change gene expression, and then move once again. Dynamics and positioning are crucial aspects of immune function that need to be described and understood if we want to have an accurate picture of the system and how it carries out its activities.

Like a watchmaker, who uses magnifying lenses to peer at the minute parts of a complex timepiece to check its function, investigators have turned to optical imaging to gain knowledge about the dynamic properties of immune cells in their in vivo environments. Over the past decade, in particular, intravital 2-photon imaging has provided a wealth of insights into what is now called "immunodynamics" [1-3]. We have seen how naïve $\mathrm{T}$ and $\mathrm{B}$ cells move within secondary lymphoid tissues and acquire antigenic information [4-14], the intricate dance of $\mathrm{T}$ and $\mathrm{B}$ cells at the T/B-border and within germinal centers [15-20] and of developing thymocytes in that organ $[21,22]$, the reactivation of memory T cells [23-25], and osteoclast, platelet, and neutrophil mobilization in the bone marrow [26-28], as well as the movement of innate and adaptive effectors in tissues such as skin [29-31], liver [32-34], central nervous system [35-37], lung [38,39], and tumors [40, 41] among others. Migration and local probing behavior of dendritic cells in diverse sites has been examined [4245]. The role of stromal elements in guiding immune cell migration has been discovered [46], the key contribution of adequate cell-cell adhesion in overcoming the dispersive migratory properties of lymphocytes and permitting effective intercellular cooperation has become clear [47], the restricted anatomical domains in secondary lymphoid organs within which some innate and adaptive immune cells migrate while awaiting evidence of host invasion have been revealed [48], and the in vivo operation of chemokines with regard to facilitating the encounter between rare cell populations delineated $[49,50]$.

While these discoveries have "animated" the field for years, we are still far from where we need to be to link information on molecules, signaling pathways, and gene regulatory events to these descriptive dynamics. New tools and techniques are required to see more for longer in larger volumes. Methods that allow simultaneous tracking of receptor signaling events and cell movement, of cytokine production and the response to these key mediators and of gene activation are essential for connecting dynamic behavior with function and differentiation. Regions of tissues currently inaccessible to our imaging platforms need to be made visible, tracking needs to occur in larger volumes to avoid loss of cells over the time span involved in their progression from resting cells to a differentiated state, many more cell types (including stromal elements such as mesenchymal cells, nerves, and vessels) need to be distinctively labeled for visualization at one time, and resolution must be increased to permit intracellular elements to be monitored. New computational tools must be developed to cope with the vast amount of data that will be generated by imaging more colors, with greater resolution, for longer times, and in larger volumes, including visualization methods that make such complex data understandable to the experimentalist. In this Viewpoint, we briefly describe the evolving methods (Fig. 1) that will contribute to overcoming these limitations and how their implementation will provide essential insights into immune function in health and disease.

\section{Challenge number $1-$ imaging depth}

Even in mice, the main experimental animal used for immune system dynamic imaging, many events that need to be visualized are hundreds of microns to millimeters from the surface of a tissue. Current fluorescence-based intravital imaging techniques can only probe the region near the surface (up to $200-300 \mu \mathrm{m}$ ) in dense 

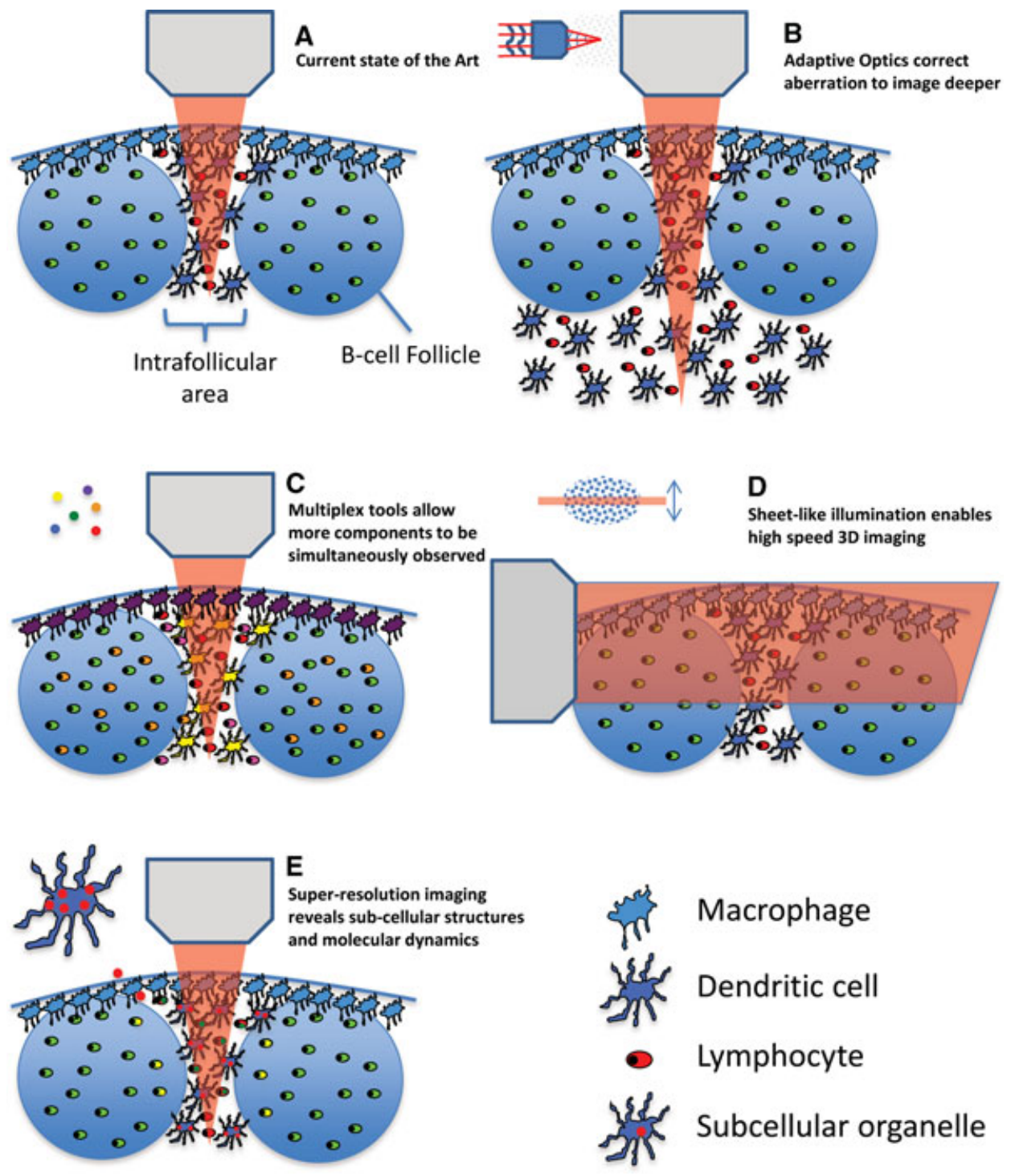

Figure 1. Overview of improved imaging modalities. (A) The current state of the art in imaging the interfollicular area and B-cell follicles of a lymph node is shown. (B) Improved imaging depth by means of adaptive optics is shown. (C) Detection of multiple different signals by means of multiplexing tools such as multiple lasers or new chromophores is shown. (D) Highspeed 3D imaging using sheet illumination is shown. (E) The visualization of subcellular compartments using super-resolution imaging techniques is shown.

lymphoid and other tissues; this is because of the dispersive optical properties of these biological structures. To gain information deep in live tissue by multiphoton imaging, photons carrying input information (excitation) have to be efficiently delivered to the location of interest (focus), and the output information (fluorescence) has to be transmitted back via emitted photons that can be collected and detected by light sensors. Within deeper areas of tissues, scattering and absorption processes will dramatically attenuate the excitation laser intensity that reaches the focal point and the heterogeneous structure of the tissue will distort the light propagation wavefront, so the excitation light cannot be tightly focused. Such optical aberration is a major factor leading to degradation of image quality.
Recognizing this limitation, many groups have been working on solutions. Near term, the simplest approach is the use of far-red or near-infrared (IR) fluorochromes and fluorescent proteins [51-54]. The longer wavelength of the excitation and emission photons involved in imaging such fluorochromes and proteins allow for better tissue penetration with less scattering or absorption than shorter wavelength photons. A penetration depth up to $1.6 \mathrm{~mm}$ in mouse cortex has been demonstrated with the laser wavelength tuned at $1.28 \mu \mathrm{m}$ and emission in the near-IR range [55].

A very promising method that is not limited by wavelength in this way involves a technique that is termed adaptive optics (AO) (Fig. 1B). Such a method aims to precompensate the light wavefront distortion inside the live tissue and allow a maximum amount of coherent laser light to reach the focal point. This approach can dramatically improve imaging contrast $[56,57]$. While the speed of the wavefront optimization process has been increased significantly, compatible with typical dynamic multiphoton intravital imaging methods, the improvement of signal quality using AO approaches comes with the price of a smaller field of view. This is because the wavefront distortion has to be measured and compensated at different locations inside the heterogeneous tissue sample, a process that takes time both due to the physical properties and software control of the adaptive mirrors employed to control the beam. These limitations will eventually be overcome through development of faster hardware and software. 
Even with current AO technology, one can now perform highly resolved imaging in small fields deep within tissues that are structurally stable (i.e. bone marrow, skin).

Other new optical approaches that extend the penetration depth even further, to beyond millimeter scale, are also in development. One such technique is ultrasound-guided optical imaging $[58,59]$, which takes advantage of an acoustic wave. The latter is less sensitive to the tissue medium that scatters the light wave and can be used to guide excitation and emission photons. This method can currently achieve imaging resolution up to $12 \mu \mathrm{m}$ at a depth of $2 \mathrm{~mm}$ [59], making it potentially useful not only for deep animal imaging but also for application to the dermis in humans, as one example.

These methods not only promise to allow visualization of objects deeper in tissues than presently accessible, but also the imaging over time of larger volumes than can be presently examined. Because of cell motility, capture of information from a larger volume not only reduces sampling error (more events can be tracked), but also permits cells to be followed over longer time intervals (because they stay within the imaging volume). In combination with hardware improvements that permit faster scanning, more sensitive photon detection, and faster movement of the stage holding the specimen, these advances will provide an enhanced ability to link early and late events during an ongoing response on a per-cell basis throughout a larger range of organ compartments. Ultimately, this will allow for continuous tracking of cells and their fates after asymmetric division and during differentiation [60].

\section{Challenge number $2-$ multiplex detection}

Immunity is a highly concerted process involving many different cellular and molecular players. For this reason, the present imaging methods that typically involve three to four colors per experiment are incapable of revealing many of the elements that play an important role in immune processes. This limitation applies at the macroscale (insufficient diversity of cell types and stromal structures visualized), so that the impact of tissue organization and many different types of cellcell contacts is not well appreciated. It is also true at the nanoscale - without an ability to multiplex more extensively, we cannot track both cell behavior and the molecules involved in cell interactions and signaling or gene activity. Yet, such comeasurements are critical if we are to link bulk dynamics to the processes of cellular activation and response crucial to immune function. Achieving these goals requires new experimental capability to observe multiple different cellular and molecular players at the same time, in the same sample, and with the same imaging configuration.

The current spectral range of fluorescence detection is from near ultraviolet (UV) to near IR. Considering the intrinsic fluorescent bandwidth of typical chromophores, the number of different fluorophores that can be distinguished within this spectral range is limited using conventional filtration methods, even assuming that an ideal set of labels with well-separated fluorescent spectra can be employed experimentally. The following four methods promise to overcome these present limitations by facilitating the simultaneous detection of multiple components (Fig. 1C):

(i) The use of multiple lasers tuned to different excitation wavelengths. Employed in the proper way, this allows optimized excitation of a variety of fluorochromes rather than the severe compromise typical of singlelaser instruments, permitting substantial improvement in detection of labels expressed at low levels and the use of more distinct fluorochromes that in combination would not be well excited with single-laser systems $[61,62]$.

(ii) The development of new chromophores (synthetic dyes or fluorescent proteins) with fluorescence spectra beyond the current range. For example, more near-IR or IR fluorescent proteins have already been developed in the past few years [51, 63-66], extending the palette available for imaging studies with current microscope systems.

(iii) The application of spectral unmixing strategies. Rather than using filters to isolate distinct (nonoverlapping) regions of emission spectra to identify targets, one can "deconvolve" the entire emitted fluorescent spectrum to identify the target chromophore by its specific emission spectral profile [67]. This requires the chromophore to have a signal strong enough to be split into the multiple spectral windows, each of which captures fewer total emitted photons. One has to find a balance between the spectral precision (more windows) and sensitivity (signal/noise $=$ larger windows), setting a limit to the use of this approach when the cell or molecule of interest can only have a limited overall fluorescent output. A related approach is to label each target component with multiple chromophores and modulate the ratio between different chromophores to identify various biological components (spectral painting). This approach has been successfully demonstrated in the application of single molecule mRNA FISH [68] and in Brainbow [69] and similar transgenic animals. Here, the complex emission spectrum from each different color combination provides a signature for that target. This approach can be further enhanced by careful choice of chromophores with distinct absorption spectra permitting selective excitation at different laser wavelengths when using multiline instruments.

(iv) The combination of standard wavelength detection methods with other strategies for fluorochrome identification. One well-established approach in single-cell imaging is fluorescence lifetime measurement [70, 71] and this has recently been incorporated into multiphoton intravital imaging [72]. In this method, special detectors and software statistically characterize the time interval (on a nanosecond scale) between the excitation and emission events for each chromophore molecule. Many spectrally overlapped chromophores have very distinct fluorescence lifetimes, so this technique can be used to add another dimension to the "identity space" of these labels. With the newest generation of high sensitivity detectors on commercial microscopes, implementation of fluorescence lifetime measurement is becoming an available tool for intravital multiphoton imaging.

A final area in which substantial progress is needed in the area of multiplex detection is in the creation of optimized probes for the analysis of intracellular signaling, molecular localization, or gene expression. A few intravital studies have characterized such events, most 
involving dye-based calcium sensors [13, 73], in vivo staining [74], fluorescent chimeric proteins $[75,76]$, and fluorescent gene reporters [77-80]. The calcium studies are limited by the leak rate of the sensor dyes to just an hour or two after cell transfer, the chimeric proteins are both difficult to detect and the analysis suffers from artifacts of optical resolution limitations and signal intensity differences in the axial dimension, and the gene reporters produce cytoplasmic proteins whose lifetime greatly exceeds that of cytokine transcripts, thus failing to provide a properly time-resolved record of gene activity that can be linked to cell dynamics [81]. To make progress in these areas, these limitations must be overcome. New fluorescent proteins have been developed [82] for the generation of optimized FRET sensors that can detect signaling events such as calcium elevation, MAPK pathway activation, and the like and should allow creation of genetically labeled cells whose signaling can be tracked without the present posttransfer time limitation. The improved sensitivity of newer instruments will permit better detection of chimeric proteins expressed close to physiologic levels and some of the methods described below will enhance axial resolution that presently limits such analyses. The creation of a new generation of genetic reporters producing destabilized fluorescent reporters, or secreted versions of such proteins, will improve the temporal connectivity between appearance of these labeled proteins and the underlying genetic activities of the cell of interest [83]. Together, such tools will allow investigators to link molecular events with cell behavior.

\section{Challenge number 3 - faster imaging}

The complexity of biological systems not only exists in the space domain, but also in the time domain. Cellular and molecular dynamics have multiplex temporal features ranging from femtoseconds to hours. Both the sampling speed and length of image collection of present instruments and their linked computers dictate the time resolution that can be achieved.

To date, the predominant scheme for sample illumination is point scanning, which limits information collection rate due to the time needed to move the beam over the entire $x-y$ dimensions of the imaging field. A major breakthrough in the past few years allows for parallel illumination of biological samples, sometimes in three dimensions. In these methods, a modified optical system illuminates a selected plane rather than a single point inside the biological sample, with the fluorescence from the whole plane detected by a wide field imager (Fig. 1D). For example, light sheet microscopy [84-86] uses one lens to create a narrow sheet of laser focus inside the tissue, and another objective is used to acquire fluorescent images at the perpendicular direction. Bessel beambased light sheet microscopy [87] further improves the spatial resolution especially in the axial dimension. Another temporal focusing scheme [88, 89] modulates the excitation laser pulse width so that the multiphoton excitation is confined in a sheet-like region. Although the temporal focusing method is limited to nonlinear excitation, it brings the convenience of using only a single objective lens with less spatial restriction for sample arrangement.

\section{Challenge number 4 - higher resolution}

In principle, the resolution of an optical microscope is limited by the optical diffraction, in the range of a few hundred nanometers with a lens of the highest possible numerical aperture. In practice, one has to find a balance between the working distance of the lens and numerical aperture, preventing use of the best resolving lenses for most intravital imaging purposes. In addition, the optical aberration inside tissues also degrades resolution, so methods to correct such aberrations, like the AO approaches discussed above, are needed to achieve near-diffraction-limit resolution deep inside tissues.

During the past decade, however, there have been a number of exciting breakthroughs that allow biological samples to be imaged at a resolution far beyond the optical diffraction limit (Fig. 1E). These novel approaches can be generalized into two types: one type takes advantage of single molecule location measurement, such as photoactivated localization microscopy, fluorescence photoactivated localization microscopy, and stochastic optical reconstruction microscopy [90-92]; the other creates subdiffraction excitation patterns, such as stimulated emission depletion [93] and structured illumination [94]. These new techniques are poised to reveal detailed molecular and structural information inside live tissues. Although to date most applications are with relatively flat cells in culture systems, some exciting recent developments have begun to incorporate such schemes into intravital imaging scenarios [95], permitting the detection of subcellular events with improved precision, at least close to the surface of various tissues [96].

\section{Challenge number 5 - data cap- ture and processing}

All the above advances will help collect more information on the immune system in situ, but without the proper means for data analysis, we will make little progress in understanding things. A new generation of software tools is needed to better analyze the image data, preferably allowing more to be done automatically and in an unbiased manner than is presently possible [97]. Some progress has been made in this arena over the past several years, both by commercial software vendors and academic centers, but much more is needed. Optimized algorithms are required to handle the much larger number of objects to be tracked when the imaging volume is increased and more cell types are visualized in more colors, for both analytic purposes and for display of the underlying cell movements; embryologists have made substantial progress in this direction $[86,98]$ and their methods need to be adopted (and adapted) by immunological imaging experts. Progress is also needed to address specific issues in molecular imaging, which is highly sensitive to depth-related image intensity artifacts. Some schemes to deal with such problems have already appeared [75, 79] but as the field embraces molecular imaging going forward, further improvements will be crucial. Other analytic tools that have been introduced to handle the analysis of cell movements into and out of defined volumes such as germinal centers or to measure synaptic dimensions $[99,100]$, provide a display in two dimensions out of four or more parameters such as time, speed, directionality, and distance from a defined site. These parameters greatly aid in understanding chemosensing behavior (Lämmermann et al., submitted manuscript), differentiate random walk from other migratory behavior [101, 102], and evaluate contact times between two cell types [103]. These datasets may then be used to derive mathematical models of complex cellular behavior, which by iterative processes can be refined and 
may allow for prediction of biological outcomes that can be tested in in vivo models [102,104-110]. Finally, the field will need to move away from the maximum projection of the 2D movies that are currently used for 3D displays that allow a better appreciation of depth in the full imaging volume.

\section{Conclusion}

Here, we have very briefly surveyed some of the emerging techniques that will aid our probing of the dynamic behavior of the immune system going forward. They will allow a larger portion of an organ to be examined, many more elements to be tracked simultaneously, cell analysis to be combined with molecular imaging, and cell function linked to dynamic behavior. An essential point to emphasize, however, is that while such imaging can be revealing in its own right, it is most valuable as a part of the larger fabric of immune investigation; dynamic imaging data need to be properly associated with information gathered by other means, such as static tissue imaging $[111,112]$, flow cytometry, ex vivo assessment of lymphocyte activity and polarization, genetic and epigenetic studies, and overall measurements of systemic immunity and host resistance. Only through integration of information garnered using the full range of methods available to the field can we develop a comprehensive model of immune system behavior, in which events on the micro- and macroscale are linked to the mesoscale dynamics of individual cells that are the present focus of imaging analysis. With the increased depth and breadth of analysis, we anticipate from rigorous application of the methods reviewed here, we are confident the future is, not to put too fine a point on it, "bright".

Acknowledgments: This work was supported by the Intramural Research Program of NIAID, NIH. We apologize to all colleagues whose primary work could not be directly cited due to editorial length and reference limitations.

Conflict of interest: The authors declare no financial or commercial conflict of interest.

\section{References}

1 Germain, R. N. et al., Science 2012. 336: 16761681.
2 Victora, G. D. et al., Annu. Rev. Immunol. 2012. 30: 429-457.

3 Pittet, M. J. et al., Cell 2011. 147: 983-991.

4 Miller, M. J. et al., Science 2002. 296: 18691873.

5 Stoll, S. et al., Science 2002. 296: 1873-1876.

6 Lindquist, R. L. et al., Nat. Immunol. 2004. 5: 1243-1250.

7 Suzuki, K. et al., J. Exp. Med. 2009. 206: 14851493.

8 Phan, T. G. et al., Nat. Immunol. 2007. 8: 9921000.

9 Carrasco, Y. R. et al., Immunity 2007. 27: 160171.

10 Junt, T. et al., Nature 2007. 450: 110-114.

11 Mempel, T. R. et al., Nature 2004. 427: 154 159.

12 Hickman, H. D. et al., Nat. Immunol. 2008. 9: 155-165.

13 Qi, H. et al., Science 2006. 312: 1672-1676.

14 Bousso, P. et al., Nat. Immunol. 2003. 4: 579585.

15 Victora, G. D. et al., Cell 2010. 143: 592-605.

16 Qi, H. et al., Nature 2008. 455: 764-769.

17 Okada, T. et al., PLoS Biol. 2005. 3: 1047-1061.

18 Allen, C. D. et al., Science 2007. 315: 528-531.

19 Schwickert, T. A. et al., Nature 2007. 446: 8387.

20 Gunzer, M. et al., Blood 2004. 104: 2801-2809.

21 Bousso, P. et al., Science 2002. 296: 1876-1880.

22 Le Borgne, M. et al., Nat. Immunol. 2009. 10: 823-830.

23 Cavanagh, L. L. et al., Nat. Immunol. 2005. 6: 1029-1037.

24 Sung, J. H. et al., Cell 2012. 150: 1249-1263.

25 Kastenmuller, W. et al., Immunity 2013. 38:502-513.

26 Kohler, A. et al., Blood 2011. 117: 4349-4357.

27 Ishii, M. et al., Nature 2009. 458: 524-528.

28 Junt, T. et al., Science 2007. 317: 1767-1770.

29 Celli, S. et al., Nat. Med. 2011. 17: 744-749.

30 Peters, N. C. et al., Science 2008. 321: 970-974.

31 Gebhardt, T. et al., Nature 2011. 477: 216-219.

32 Egen, J. G. et al., Immunity 2011. 34: 807-819.

33 McDonald, B. et al., Science 2010. 330: 362366.

34 Geissmann, F. et al., PLoS Biol. 2005. 3: e113.

35 Wilson, E. H. et al., Immunity 2009. 30: 300311.

36 Kawakami, N. et al., J. Exp. Med. 2005. 201: 1805-1814.

37 Kim, J. V. et al., Nature 2009. 457: 191-195.

38 Kreisel, D. et al., Proc. Natl. Acad. Sci. USA 2010. 107: 18073-18078.

39 Bruns, S. et al., PLoS Pathog. 2010. 6: e1000873.

40 Breart, B. et al., J. Clin. Invest. 2008. 118: 13901397.

41 Boissonnas, A. et al., J. Exp. Med. 2007. 204: 345-356.

42 Chieppa, M. et al., J. Exp. Med. 2006. 203: 2841-2852.
43 McDole, J. R. et al., Nature 2012. 483: 345-349.

44 Thornton, E. E. et al., J. Exp. Med. 2012. 209: 1183-1199.

45 Miller, M. J. et al., Proc. Natl. Acad. Sci. USA 2004. 101: 998-1003.

46 Bajenoff, M. et al., J. Immunol. 2008. 181: 3947-3954.

47 Schneider, H. et al., Science 2006. 313: 1972 1975.

48 Kastenmuller, W. et al., Cell 2012. 150: 12351248.

49 Castellino, F. et al., Nature 2006. 440: 890-895.

50 Hugues, S. et al., Nat. Immunol. 2007. 8: 921930.

51 Filonov, G. S. et al., Nat. Biotechnol. 2011. 29: 757-761.

52 Lin, M. Z. et al., Nat. Methods 2011. 8: 726-728.

53 Mojzisova, H. et al., Curr. Opin. Genet. Dev. 2011. 21: 549-557.

54 Shcherbo, D. et al., Nat. Methods 2010. 7: 827829.

55 Kobat, D. et al., J. Biomed. Opt. 2011. 16: 106014.

56 Ji, N. et al., Nat. Methods 2010. 7: 141-147.

57 Tang, J. et al., Proc. Natl. Acad. Sci. USA 2012. 109: 8434-8439.

58 Wang, Y. M. et al., Nat. Commun. 2012. 3: 928.

59 Si, K. et al., Sci. Rep. 2012. 2: 748.

60 Chang, J. T. et al., Science 2007. 315: 16871691.

61 Mahou, P. et al., Nat. Methods 2012. 9: 815818.

62 Entenberg, D. et al., Nat. Protoc. 2011. 6:15001520.

63 Hilderbrand, S. A. et al., Curr. Opin. Chem. Biol. 2010. 14: 71-79.

64 Luo, S. et al., Biomaterials 2011. 32: 7127-7138.

65 Andresen, V. et al., Curr. Opin. Biotechnol. 2009. 20: 54-62.

66 Subach, F. V. et al., Nat. Methods 2011. 8: 1019-1026.

67 Zimmermann, T. et al., Adv. Biochem. Eng. Biotechnol. 2005. 95: 245-265.

68 Femino, A. M. et al., Science 1998. 280: 585590.

69 Livet, J. et al., Nature 2007. 450: 56-62.

70 Dong, C. Y. et al., Methods Cell Biol. 2003. 72: 431-464.

71 French, T. et al., Methods Cell Biol. 1998. 56: 277-304.

72 Fruhwirth, G. O. et al., Proc. SPIE 2009. 7183: 71830L-71830L.

73 Bhakta, N. R. et al., Nat. Immunol. 2005. 6: 143-151.

74 Schwendele, B. et al., Eur. J. Immunol. 2012. 42: 2193-2196.

75 Friedman, R. S. et al., J. Exp. Med. 2010. 207: 2733-2749.

76 McCombs, J. E. et al., Methods 2008. 46: 152159

77 Hoffman, R. M. et al., Curr. Pharm. Biotechnol. 2012. 13: 537-544.

78 Giepmans, B. N. et al., Science 2006. 312: 217224. 
79 Melichar, H. J. et al., Immunol. Cell Biol. 2011. 89: 549-557.

80 Croxford, A. L. et al., Immunology 2011. 132: 1-8.

81 Beuneu, H. et al., Immunity 2010. 33: 412-423.

82 Lam, A. J. et al., Nat. Methods 2012. 9: 10051012.

83 Houser, J. R. et al., Yeast 2012. 29: 519-530.

84 Keller, P. J. et al., Science 2008. 322: 1065-1069.

85 Huisken, J. et al., Science 2004. 305: 10071009.

86 Truong, T. V. et al., Nat. Methods 2011. 8: 757760.

87 Planchon, T. A. et al., Nat. Methods 2011. 8: 417-423.

88 Durst, M. E. et al., Opt. Commun. 2008. 281: 1796-1805.

89 Oron, D. et al., Opt. Express 2005. 13: 14681476.

90 Betzig, E. et al., Science 2006. 313: 1642-1645.

91 Hess, S. T. et al., Biophys. J. 2006. 91: 42584272.

92 Rust, M. J. et al., Nat. Methods 2006. 3: 793 795.

93 Hell, S. W. et al., Opt. Lett. 1994. 19: 780-782.

94 Gustafsson, M. G. et al., Biophys. J. 2008. 94: $4957-4970$
95 York, A. G. et al., Nat. Methods 2011. 8: 327333.

96 York, A. G. et al., Nat. Methods 2012. 9: 749754.

97 Ludewig, B. et al., Eur. J. Immunol. 2012. 42: 3116-3125.

98 Tomer, R. et al., Nat. Methods 2012. 9: 755763.

99 Klauschen, F. et al., Nat. Protoc. 2009. 4: 13051311.

100 Klauschen, F. et al., Nat. Protoc. 2009. 4: 10061012.

101 Harris, T. H. et al., Nature 2012. 486: 545-548.

102 Beltman, J. B. et al., J. Exp. Med. 2007. 204: 771-780.

103 Dustin, M. L. et al., Proc. Natl. Acad. Sci. USA 1997. 94: 3909-3913.

104 Garin, A. et al., Immunity 2010. 33: 84-95.

105 Figge, M. T. et al., J. Exp. Med. 2008. 205: 30193029.

106 Bogle, G. et al., PLoS One 2012. 7: e45258.

107 Beltman, J. B. et al., J. Immunol. Methods 2009. 347: 54-69.

108 Beltman, J. B. et al., Immunol. Cell Biol. 2007. 85: 306-314.

109 Bogle, G. et al., Immunol. Cell Biol. 2010. 88: 172-179.

110 Mandl, J. N. et al., Proc. Natl. Acad. Sci. USA 2012. 109: 18036-18041.
111 Gerner, M. Y. et al., Immunity 2012. 37 364-376.

112 Moreau, H. D. et al., Immunity 2012. 37: 351-363.

Correspondence: Dr. Ronald N. Germain, Laboratory of Systems Biology, National Institute of Allergy and Infectious Diseases, National Institutes of Health, Bldg. 4, Rm. 126A MSC-0421, 10 Center Drive, Bethesda, MD 20892-1892, USA

Fax: +1-301-480-1660

e-mail: rgermain@nih.gov

Received: 5/11/2012

Revised: 1/3/2013

Accepted: 3/4/2013

Keywords: 2-photon - Dynamic - Imaging • Immune responses $\cdot$ Multicolor

Abbreviations: AO: adaptive optics · IR: infrared

The complete Cells in Motion Viewpoint series is available at: http://onlinelibrary.wiley.com/doi/10. 1002/eji.v43.6/issuetoc

\title{
Molecules regulating migration
}

\section{Alarmin-induced cell migration}

\author{
De Yang ${ }^{1,2,3}$, Feng Wei ${ }^{2,3}$, Poonam Tewary ${ }^{2}$, O. M. Zack Howard ${ }^{2}$ and Joost J. Oppenheim ${ }^{2}$ \\ ${ }^{1}$ Basic Research Program, Scientific Application and International Corporation-Frederick, \\ Inc., Frederick, MD, USA \\ ${ }^{2}$ Laboratory of Molecular Immunoregulation, Cancer and Inflammation Program, \\ National Cancer Institute, Frederick National Laboratories for Cancer Research, \\ Frederick, MD, USA \\ ${ }^{3}$ Research Center of Basic Medical Sciences, Tianjin Medical University, Tianjin, China
}

DOI: $10.1002 /$ eji.201243138

Alarmins are endogenous, constitutively available, damage-associated molecular patterns that upon release can mobilize and activate various leukocytes for the induction of innate and adaptive immune responses. For our immune system to function appropriately, it relies on navigating various leukocytes to distinct places at the right time. The direction of cell migration is determined by chemotactic factors that include classical chemoattractants, chemokines, certain growth factors, and alarmins. This viewpoint provides an overview of alarmininduced cell migration. Alarmins are capable of inducing the migration of diverse types of leukocytes and nonleukocytes either directly by triggering specific receptors or indirectly by inducing production of chemokines through the activation of various leukocytes via pattern recognition receptors. The receptors used by alarmins to directly induce cell migration can either be Goi proteincoupled receptors or receptors such as the receptor for advanced glycation end products; however, the intracellular signaling events responsible for the direct chemotactic activities of alarmins are, to date, only partially elucidated. Given that alarmins act in concert with chemokines to regulate the recruitment and trafficking of leukocytes, these damageassociated molecular patterns are potentially involved in diverse 
biological processes as discussed in this viewpoint.

\section{Introduction}

The migration of single cells is important for many physiologic processes such as ontogenic development, organogenesis, hematopoiesis, tissue regeneration, and immune responses. Cell migration is also involved in pathological conditions such as autoimmune disorders, vascular disease, and tumor metastasis. The ability of the immune system to respond appropriately to microbial invasion, tissue damage, and other insults relies to a large extent on the mobilization/recruitment of various leukocytes and progenitor cells to the right place at the right time [1,2]. Migration of cells in vivo is controlled by many sequential interactions involving adhesion molecules, glycosaminoglycans, chemotactic factors, and their receptors [1-5].

For cells to move directionally, they must first acquire a polarized morphology where F-actin is primarily enriched at the front and myosin II is assembled on the sides and at the back of the cell [6]. Subsequently, the polarized cells undergo a highly coordinated cycle of protrusions and retractions that are coupled with traction provided by the formation and release of adhesive contacts with the extracellular matrices [6]. Cells must be able to determine where and when protrusions, retractions, and adhesions have to occur to migrate to the correct location, which is established by chemotactic gradients. Chemotactic factors are comprised of classical chemoattractants such as formyl peptides and anaphylatoxins (e.g. C5a), chemokines including CXC, CC, CX3C, and $\mathrm{XC}$ chemokines, and growth factors such as EGF and vascular endothelial growth factor $[1,4,7,8]$. Classical chemoattractants and chemokines provide extracellular cues by signaling predominantly through $\mathrm{G} \alpha \mathrm{i}$ protein-coupled receptors (GiPCRs), while growth factors do so by signaling through their corresponding receptors $[4,7,8]$.

A more recently identified type of chemotactic factor is the alarmin family. Alarmins are structurally distinct endogenous mediators that, upon release and gaining access to immune cells, can activate the immune system by inducing the recruitment and activation of various leukocytes, particularly APCs, including DCs [9-11]. Consequently, alarmins are capable of inducing both innate and antigen-specific host immune responses. Most alarmins are constitutively expressed and stored in intracellular compartments such as the nucleus, cytoplasm, or granules. The expression of some alarmins can also be upregulated by microbial products, cytokines, and stress. During microbial infection and/or tissue injury, alarmins rapidly become available extracellularly as a result of degranulation, passive release due to cell necrosis, or active release in response to inducing agents [9-11].

Known alarmins are multifunctional and can be classified into eight distinct molecular categories (Table 1), including defensins (e.g. $\alpha$ - and $\beta$-defensins), cathelicidin (e.g. human LL-37 or mouse cathelicidin-related antimicrobial peptide (CRAMP)), eosinophil-associated ribonucleases (e.g. eosinophil-derived neurotoxin (EDN)), nuclear-binding proteins (e.g. high-mobility group box-1 protein (HMGB1), high-mobility group nucleosome-binding protein 1 (HMGN1), and the cytokines IL-1 $\alpha$ and IL-33), HSPs

Table 1. Target cells and receptors of alarmin-induced cell migration.

\begin{tabular}{|c|c|c|c|c|}
\hline \multicolumn{2}{|l|}{ Alarmin } & \multirow[t]{2}{*}{ Target cell } & \multicolumn{2}{|l|}{ Receptor } \\
\hline Category & Member & & GiPCR & Non-GiPCR \\
\hline \multirow[t]{2}{*}{ Defensin } & $\alpha$-Defensin family & $\begin{array}{l}\mathrm{MC}[21], \mathrm{Mo} / \mathrm{M} \phi[16,21], \mathrm{DC} \\
\quad[17], \mathrm{T}[15,17,21]\end{array}$ & n.d. & n.d. \\
\hline & $\beta$-Defensin family & $\begin{array}{l}\text { MC [22], Mo/M } \phi[26,27], \text { DC } \\
{[18,19,23,28], \mathrm{T}[18], \mathrm{EPC}} \\
{[37,70], \mathrm{Ep}[75]}\end{array}$ & $\begin{array}{l}\text { CCR6 }[18-20,23,70] \\
\text { CCR2 }[26,27]\end{array}$ & EGFR [75] \\
\hline Cathelicidin & LL-37/CRAMP & $\begin{array}{l}\text { PMN [32], MC [33, 34], Mo [32], } \\
\text { DC [38], T [32], MSC }[35,36] \\
\text { Ep }[35,37]\end{array}$ & $\begin{array}{l}\text { FPRL1/FPR2 } \\
{[32,35,36,38], \operatorname{MrgX} 2} \\
{[34]}\end{array}$ & EGFR [37] \\
\hline EAR & EDN/EAR2 & $\mathrm{DC}[40]$ & n.d. & n.d. \\
\hline \multirow[t]{3}{*}{ Nuclear-binding protein } & HMGB1 & $\begin{array}{l}\text { PMN [50], M } \phi[51], \mathrm{DC}[52] \\
\quad \text { MSC [53-55], EPC [56], SMC } \\
\quad[57,58], \text { Ep [59,60] }\end{array}$ & CXCR4 $[89,99]$ & $\begin{array}{l}\text { RAGE [50-53, } \\
56,72,73]\end{array}$ \\
\hline & HMGN1 & Mo, DC [49] & n.d. [59] & n.d. \\
\hline & IL-33, IL-1 $\alpha$ & PMN [64], T [63] & n.d. & ST2 [63] \\
\hline HSP & HSP60, 70 & DC, NK $[41]$ & n.d. & n.d. \\
\hline Saposin-like & Granulysin & Mo [39], DC [42], T [39] & n.d. & n.d. \\
\hline \multirow[t]{2}{*}{ Ion-binding protein } & $\mathrm{S} 100 \mathrm{a} 7,8,12,15$ & $\begin{array}{l}\mathrm{PMN}[43,47], \mathrm{MC}[44], \mathrm{Mo} / \mathrm{M} \phi \\
\quad[46,47]\end{array}$ & n.d. & RAGE [46] \\
\hline & Lactoferrin & $\mathrm{Mo} / \mathrm{M} \phi[45]$ & n.d. & n.d. \\
\hline \multirow[t]{2}{*}{ Nucleotide/metabolite } & ATP & $\begin{array}{l}\mathrm{PMN}, \mathrm{Eo}, \mathrm{Mo} / \mathrm{M} \phi, \mathrm{DC}, \mathrm{EC}, \mathrm{SMC} \\
{[66]}\end{array}$ & P2Y2, P2Y6, P2Y12 [66] & $\mathrm{P} 2 \mathrm{X} 7[66]$ \\
\hline & Uric acid & Eo $[65]$ & n.d. & n.d. \\
\hline
\end{tabular}

CCR: CC chemokine receptor; EAR: eosinophil-associated ribonuclease; EC: endothelial cell; Ep: epithelial cell; FPRL1: formyl peptide receptorlike 1 receptor; M $\phi$ : macrophage; Mo: monocyte; MrgX2: Mas-related gene X2; MSC: mesenchymal stem/stromal cell; PMN: polymorphonuclear neutrophil; T, T cell. n.d. = not determined. 
(e.g. HSP60, HSP70), saposin-like granulysin, ion-binding proteins (e.g. lactoferrin, S100 proteins), and nucleotides/ metabolites (e.g. ATP, uric acid). Several alarmins, including HMGB1, S100A8/9, ATP, and uric acid, were found to not only play a chemotactic role but to also function as damage-associated molecular patterns (DAMPs) since they are released as a result of cell injury/death and can perpetuate immune responses [10-12]. The term DAMP was proposed in 2004 to designate hyppos (biological molecules with hydrophobic portions) capable of initiating repair, remodeling, and immune responses [12]. DAMPs are broadly defined and include both endogenous molecules engaged in host defense and metabolism (HSPs, uric acid, etc.) and exogenous pathogen-associated molecular patterns such as LPS, flagellin, and bacterial DNA [12].

Given the dual roles of defensins, cathelicidins, EDN, and HMGB1, the term alarmin was coined, also in 2004 , to classify a set of endogenous mediators that possess the dual capacities of promoting host defenses against dangers by inducing the migration/recruitment and activation of APCs and consequently are capable of initiating/enhancing innate and adaptive host immune responses $[9,13]$. Alarmins, like cytokines, can be dangerous if produced in excess under inappropriate circumstances such as during severe injury, autoimmunity, and tumor progression. Since alarmins and DAMPs overlap in terms of release and immunostimulatory effect, alarmins can be considered an endogenous subset of DAMPs $[10,14]$. It has also become clear since the initial classification that alarmins are involved in the induction of cell migration, in vivo recruitment, and cell activation through multiple mechanisms.

\section{Alamins directly induce cell migration}

\section{The chemotactic effects of alarmins}

Alarmins are chemotactic for diverse types of leukocytes as well as nonleukocytes (Table 1). Both human and mouse $\alpha$ and $\beta$-defensins are chemotactic for immature DCs, monocytes/macrophages, mast cells (MCs), and certain subsets of T lymphocytes [15-27]. The leukocyte chemotactic activity of defensins appears to be universal across vertebrate species since defensins from bovine and fish species are also chemotactic $[28,29]$. Cathelicidins can chemoattract many subsets of leukocytes as well as nonleukocytes such as mesenchymal stromal cells and keratinocytes [30-38]. EDN, HSPs, granulysin, S100 proteins, and lactoferrin are chemotactic for various subsets of leukocytes [39-48]. The recently identified alarmin HMGN1 is important for the recruitment of DCs in vivo [49] and possesses direct chemotactic activity for monocytes and DCs (D. Yang et al., unpublished results). HMGB1, which belongs to the nuclear-binding protein category as does HMGN1, is a multifunctional alarmin that has been shown to induce migration of the widest spectrum of target cells, including neutrophils [50], monocytes/macrophages [51], DCs [52], mesoangioblasts [53], mesenchymal stromal cells $[54,55]$, endothelial progenitor cells (EPCs) [56], smooth muscle cells (SMCs) $[57,58]$, fibroblasts and keratinocytes [59], and certain tumor cells [60]. HMGB1 also promotes the outgrowth of neurites and the motility of neurons $[61,62]$. IL-33, a nuclearbinding protein alarmin, is reported to be chemotactic for Th2 $\mathrm{T}$ lymphocytes and neutrophils $[63,64]$. Nucleotides and their metabolites, including uric acid, are the only type of alarmins that are not protein in nature. Uric acid has thus far only been reported to induce chemotaxis of eosinophils [65], while ATP is chemotatic for many leukocytes and nonleukocytes (reviewed recently in [66]).

The chemotactic effects of alarmins on various target cells are often demonstrated in vitro by a Boyden chamber-based multiwall chemotaxis assay, a reliable method widely used for investigating the migration of cells in response to many chemotactic factors including chemokines. The capacity of various alarmins to induce in vivo cell recruitment can be demonstrated by injecting alarmin(s) into airpouch, peritoneal cavity, or solid tissue and subsequently quantifying the type and number of leukocytes attracted into the injection sites. For example, the T-cell attracting capacity of human neutrophilderived $\alpha$-defensin was confirmed by the accumulation of human CD3-positive $\mathrm{T}$ cells at the subcutaneous site $4 \mathrm{~h}$ after injection of $\alpha$-defensin in an experimental model system [15]. The in vivo capacity of HMGB1 to induce the recruitment of mesoangioblasts was determined by the migration of intraartery injected mesoangioblasts toward HMGB1-loaded heparinSepharose beads implanted in the muscle of experimental mice [53]. The capacity of EDN or cathelicidin to chemoattract APCs in vivo was demonstrated by the recruitment of DCs and macrophages into airpouches after intrapouch administration of the respective alarmins $[40,67]$. The most often used in vivo model is the peritonitis model, which has been used to verify the in vivo leukocyte-recruiting capacity of lactoferrin, granulysin, HMGB1, and HMGN1 $[42,45,49,68]$.

What appears to be common among most alarmins is the capacity to induce the migration of APCs including monoyctes, macrophages, and DCs (Table 1). It is likely that most alarmins participate in regulating the recruitment and trafficking of DCs. Since the characterization of alarmininduced cell migration is still incomplete, much more has to be elucidated before a complete picture can be painted with regard to the target cell spectrum of all alarmins.

\section{Receptors that mediate the chemotac- tic effects of alarmins}

The chemotactic cell migration induced by most alarmins can be inhibited by pretreatment of the target cells with pertussis toxin, a bacterial toxin capable of preventing $G$ proteins from interacting with GiPCRs on the cell membrane by catalyzing the ADP-ribosylation of the $\alpha_{i}$ subunits of the heterotrimeric $\mathrm{G}$ protein $[17,18$, $21,22,25,32,33,35,36,39,40,45,52,67]$. This indicates that the direct chemotactic effects of many alarmins are mediated by GiPCRs (Table 1). Some alarmins use more than one GiPCR, e.g. $\beta$-defensin 2 uses both CCR6 and CCR2 [18, 19,26,27]. Furthermore, GiPCR usage by alarmins overlaps with that of chemokines; e.g. the $\beta$-defensin 2 and 3 share CCR6 with CCL20 [18, 19, 23, 24, 69-71], while the $\beta$-defensin 3 and 14, which were more recently reported to also use CCR2 [26, 27], share CCR2 with a number of CC chemokines such as CCL2, CCL7, CCL8, and CCL12 $[1,4,7]$.

The capacity of human and mouse cathelicidin to induce the migration of most target cells is mediated by FPRL1 (FPR2 in mouse) $[32,35,36,67]$ and it has recently been shown that LL-37 induces MC migration and degranulation through MrgX2, a GiPCR belonging to Mas-related gene family [34]. ATP uses several GiPCRs for inducing the migration of various target cells, such as P2Y2 on DCs, eosinophils, and fibroblasts, P2Y6 on monocytes and P2Y12 on microglia and SMCs [66]. The 
identity of GiPCRs responsible for mediating the chemotactic effects of $\alpha$-defensins, eosinophil-associated ribonucleases, HSPs, HMGN1, granulysin, lactoferrin, and uric acid, remain to be identified (Table 1).

Receptors other than GiPCRs are also used by alarmins to induce chemotactic migration (Table 1). HMGB1-induced migration of neutrophils, monocytes, DCs, mesoangioblasts, endothelial progenitors, and glioblastoma cells is dependent on the presence of the receptor for glycation end products (RAGE) [50-53, 56, 72, 73]. While S100A8- and S100A15-induced migration of neutrophils and macrophages is mediated by GiPCRs [46-48], monocyte migration induced by S100A7 is RAGEmediated [46]. IL-33-induced cell migration appears to be mediated by ST2, an IL-1 receptor like-1 receptor $[63,74]$. The EGF receptor may mediate the cell-attracting effect of some alarmins. The migration of keratinocytes in response to LL-37 appears to be mediated by transactivation of EGF receptor [37]. The migration of keratinocytes induced by several $\beta$-defensins is also reported to involve EGF receptor transactivation in a pertussis toxinsensitive manner [75], which suggests that an as-yet-unidentified GiPCR may also be involved. The interaction between LL-37 and insulin-like growth factor 1 receptor on MCF-7, a human breast cancer cell line, also induces cell migration, suggesting that LL-37 may also use this receptor to promote the migration of certain tumor cells [76].

\section{Intracellular signaling pathways}

Several intracellular signaling cascades driving cell migration are triggered by alarmins. Cathelicidins elevate intracellular $\mathrm{Ca}^{2+}$ in neutrophils, monocytes, and MCs [30, 32, 33, 67]. LL-37 induces signaling through insulin-like growth factor 1 receptor to activate ERKs, phosphatidylinositol-3 kinase (PI3K)-Akt pathways, both of which participate in the migration and motility of fibroblasts and breast cancer cells [76]. The migration of intestinal epithelial cells induced by human $\beta$-defensin 2 is accompanied by an increase of intracellular $\mathrm{Ca}^{2+}$, activation of RhoA, and PI3K [71]. HMGB1-induced migration of glioblastoma cells, endothelial cells, and mesoangioblasts depends, at least in part, on the activation of ERKs [73, 77, 78]. HMGB1 triggers cytoskeleton reorganization in SMCs, which is necessary for their migration [57]. On the other hand, HMGB1-stimulated migration of human chondrosarcoma cells and SMCs requires the activation of the PI3K-Akt signaling pathway $[58,60]$. Defensins also induce the activation of the PI3K-Akt signaling pathway in intestinal epithelial cells in the course of promoting their migration [71]. Overall, it appears that alarmins, in the process of inducing the migration of diverse types of cells, trigger the activation of PI3K/Akt, ERKs, small GTPases (e.g. Rho, Rac, etc.), PKC, and elevation of intracellular $\mathrm{Ca}_{2}^{+}$.

For cell migration induced by alarmins, the intracellular signaling events that connect the receptor (s) and intracellular signaling messengers (e.g. PI3K/Akt, ERKs, PKC, Rho, $\mathrm{Ca}^{+}$, etc.) still need to be determined. Since the predominant receptors mediating the chemotactic effects of alarmins are GiPCRs and RAGE, and the signaling pathways of chemotactic receptors (e.g. FPRL1, CCR2, CCR6) or RAGE have been elucidated in some detail $[1,4,7,8]$, it is likely that alarmins trigger similar intracellular signaling pathways to those of chemokines and AGEs for the induction of cell migration as shown in Figure 1 . Thus binding of an alarmin with its GiPCR presumably results in the activation of heterotrimeric G-protein(s) that dissociate into activated $\mathrm{G} \alpha \mathrm{i}$ and $\mathrm{G} \beta \gamma$ subunits. Activated $\mathrm{G} \alpha \mathrm{i}$ and $\mathrm{G} \beta \mathrm{G} \gamma$ trigger a series of reactions, commencing with the activation of phospholipase C (PLC $\beta$ ), PI3K, and small GTPases (Rac and Rho) [1, 4, $7,8]$. Ligand engagement of RAGE leads to activation of PI3K [79] and Rac [80]. PLC $\beta$ hydrolyses phosphatidylinositol-4, 5-bisphosphate (PIP2), generating inositol 1,4,5-trisphosphate (IP3), and diacylglycerol. IP3 induces the release of calcium from intracellular stores, and together with diacylglycerol, activates PKC. Activation of PI3K in turn phosphorylates Akt. Rac and Rho initiates a series of reactions that lead to the activation of ERKs, LIM (named by the initials of the three homeodomain proteins Lin11, Isl-1, and Mec-3 in which it was first discovered) domain kinase (LIMK), and myosin light chain. These protein kinases act cooperatively to rearrange cytoskeleton fibers leading to cell migration (Fig. 1).

\section{Alarmins indirectly promote cell migration and recruitment}

Alarmins can activate many cell types, often by triggering a pattern recognition receptor such as TLRs [9-11, 14, 49, 81].
This leads to the production of chemotactic factors and adhesion molecules, both of which participate in promoting cell migration and/or recruitment $[1-5,8]$. For example, treatment of airway epithelial cells with human $\alpha$-defensin promotes the production of CXCL8 [82]. LL-37 treatment of endothelial cells leads to the production of CCL2 [83]. Several human $\beta$-defensins can stimulate keratinocytes to produce a number of chemokines including CXCL10, CCL2, CCL20, and CCL5 [75]. HSP70 released from heat-shocked tumor cells stimulates the production of chemokines (CXCL10, CCL2, and CCL5) that induces the infiltration of DCs and $\mathrm{T}$ cells [84]. Activation of macrophages or DCs by EDN, HMGB1, HMGN1, granulysin, S100a8/9, and uric acid leads to the production of many chemokines such as CXCL5, CXCL7, CXCL8, CXCL9, CXCL10, CXCL12, CCL1, CCL2, CCL3, CCL5, CCL7, and CCL8 [39, 42, 49,52,65, 85-89]. These chemokines, in turn, promote the migration and recruitment of cells positive for the corresponding chemokine receptors including CXCR1, CXCR2, CXCR3, CCR1, CCR2, CCR5, and CCR6. LL-37 is capable of inducing the generation of vascular endothelial growth factor, which, in turn, promotes angiogenesis by facilitating the recruitment of multipotent mesenchymal stromal cells [36].

LL-37-treated EPCs migrate better than nontreated progenitor cells to the injured area due to the upregulation of E-selectin and P-selectin glycoprotein ligand-1 [90]. LL-37 also enhances ICAM-1 expression by endothelial cells [83]. HMGB1 is capable of inducing $\alpha 5 \beta 1$ integrin expression by human chondrosarcoma cells [60]. HMGB1 not only upregulates the expression of ICAM- 1 , and the $\beta 1$ and $\beta 2$ integrins on EPCs, but it also increases the affinity of these integrins, contributing to the enhanced adhesion and recruitment of EPCs [56]. Human $\beta$-defensin 2 has recently been shown to induce the arrest of Th17 cells on inflamed endothelial cells in an ICAM-1-dependent manner [91]. Therefore, alarmins have the capacity to regulate the expression or activation of various adhesion molecules on both endothelial cells and target cells and thus can promote the migration of target cells.

Overall, alarmins can induce cell migration directly through interacting with their GiPCRs or RAGE, or indirectly by stimulating the production of chemokines, growth factors, and adhesion molecules often via activating the corresponding pattern recognition receptor (Fig. 1). The 


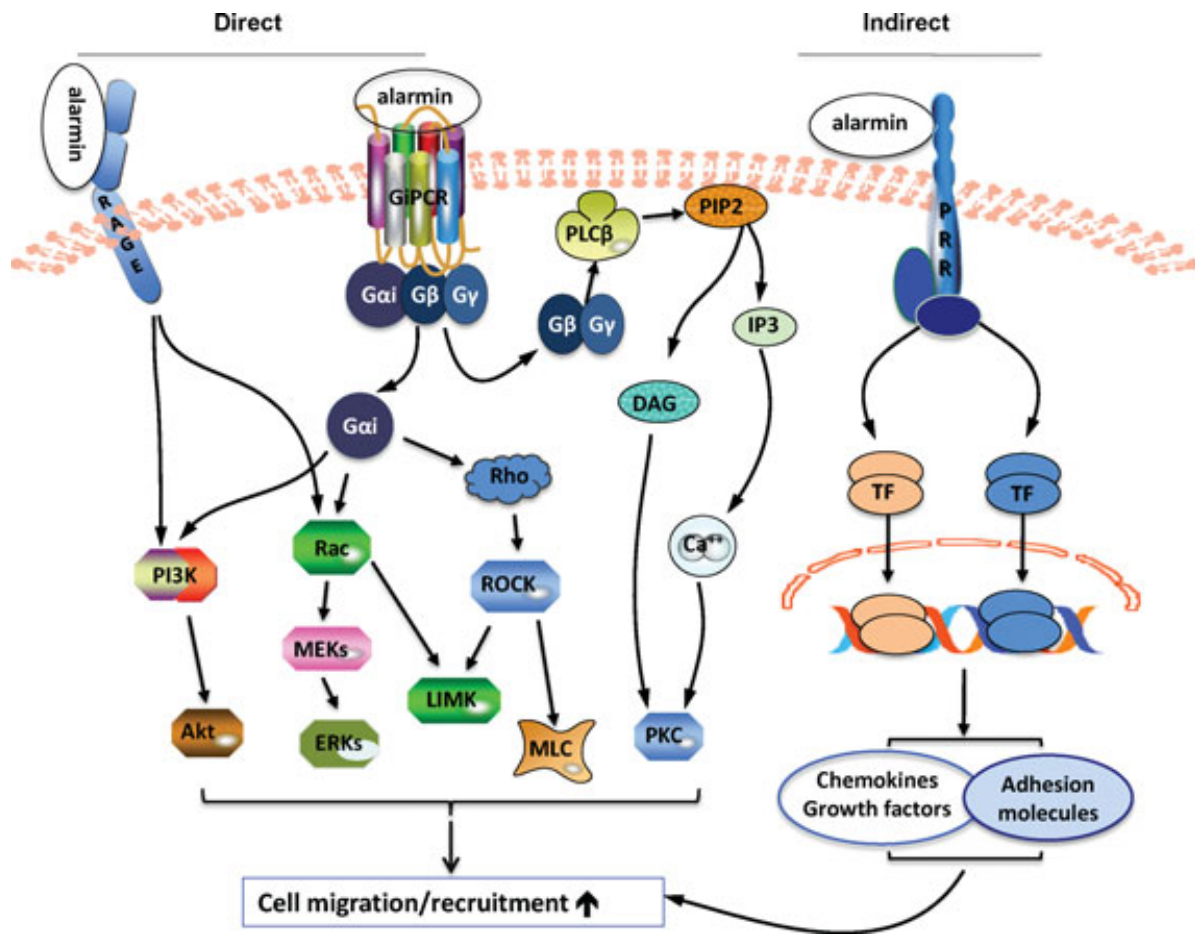

Figure 1. Pathways of alarmin-induced cell migration/recruitment. Alarmins can directly induce cell migration by engaging either a GiPCR or RAGE (left), or indirectly by stimulating the production of chemokines, growth factors, and adhesion molecules (right). Alarmin engagement with its GiPCR would lead to the activation and dissociation of heterotrimeric $G$ proteins into $G \alpha i$ and $G \beta \gamma$ subunits, which in turn, trigger the activation of PLC $\beta$, PI3K, Rac, and Rho. PLC $\beta$ hydrolyses PIP2, generating IP3 and diacylglycerol. IP3 elevates intracellular calcium, which together with diacylglycerol activates PKC. Activation of PI3K in turn phosphorylates Akt. Rac and Rho initiates a series of reactions that lead to the activation of ERKs, LIMK, and myosin light chain. Ligand engagement of RAGE leads to activation of PI3K and Rac, and subsequent activation of Akt, ERKs, and LIMK. The kinases (Akt, ERKs, LIMK, MLC, and $\mathrm{PKC}$ ) work cooperatively to rearrange cytoskeleton fibers, and ultimately to enable cell migration. mechanism used by a given alarmin depends on the type of target cell involved. It is also likely that a given alarmin induces the in vivo trafficking of target cells by more than one mechanism. A good example would be LL-37-induced migration and activation of phagocytes, MCs, DCs, and epithelial cells. LL-37/CRAMP induces the migration of neutrophils and monocytes through the use of FPRL1/FPR2 $[32,67]$, however, its capacity to stimulate the migration of MCs is mediated by MrgX2 [34]. In contrast, LL-37 can transactivate EGFR leading to the activation of ERKs and production of IL-8 [92], which would in turn promote the recruitment of neutrophils by the use of CXCR1 and CXCR2. Furthermore, LL-37 also forms complexes with DNA or RNA to promote the activation of DCs by triggering TLR7, TLR8, and TLR9, which contributes to the recruitment of inflammatory cells during psoriasis and skin wound healing $[93,94]$. Thus, alarmins presumably promote the migration and recruitment of diverse types of cells via multiple mechanisms.

\section{Biological implications}

What is the relationship between alarmins and other chemotactic factors? For example, many alarmins can induce the migra- tion of DCs (Table 1) as can many classical chemoattractants and chemokines [7, 95, 96]. Knockout of FPR2, a GiPCR for LL-37/ CRAMP [32,67], impairs the recruitment of DCs to the site of inflammation [38], suggesting a nonredundant contribution of cathelicidin to DC recruitment. Knockout of HMGN1 greatly reduces DC accumulation at the site of immunization [49]. The recruitment of DC precursors to the skin is dependent on CCR2, indicating the importance of the chemokine CCL2 in the process [97]. Both CCL20 and many defensins use CCR6 as the receptor to induce the migration of immature DCs $[7,18,19,70,95,96,98]$. Therefore, both alarmins and chemokines potentially contribute to migrational navigation of immature DCs from the blood to sites of inflammation and antigen presence. In addition, HMGB1 can form complexes with CCL12, which induces DC migration by triggering CXCR4 and RAGE [89, 99]. Mature DC homing to draining lymph nodes requires not only HMGB1-RAGE [100], but also chemokines including CCL19, CCL21, and CXCL12 [7, 95-97]. Thus, alarmins are likely to act in concert with other chemotactic factors to regulate the in vivo recruitment of various types of leukocytes.

The capacity of alarmins to induce cell migration and recruitment plays important roles in many biological processes.
HMGB1-promoted skin wound repair depends on its capacity to chemoattract fibroblasts and keratinocytes [59], as well as in vivo recruitment of keratinocytes [72]. HMGB1 promotes angiogenesis by promoting the migration of endothelial cells [77]. HMGB1, HMGN1, EDN, uric acid, S100A8/9, cathelicidin, and HSPs have all been shown to be important for the induction of inflammation and immune responses [49, 81, 84, 101-106], which is likely contributed by the capacities of these alarmins to both mobilize and activate leukocytes. HMGN1-induced DC recruitment is responsible at least in part for the capacity of HMGN1 to induce immune responses, since HMGN1 ${ }^{-/-}$mice manifest remarkably reduced immune responses accompanied by greatly reduced recruitment of DCs to the site of immunization [49]. Thus the capacity of alarmins to induce the recruitment of immune cells is critical for their participation in host defense responses. Consequently, inhibition of the release or activity of HMGB1 can ameliorate many inflammatory conditions (reviewed recently [107]). For example, blockade of HMGB1 upon heart transplantation also reduces infiltration of leukocytes and anti-graft Th1 immune responses, and significantly prolongs allograft heart survival [108]. 


\section{Perspective}

Although much has been learned in the past decade about the capacities of alarmins to induce cell migration and recruitment, much more needs to be done in order to elucidate the intracellular signaling pathways utilized by various alarmins; of note, the receptors for many alarmins remain unidentified. It is also critical to dissect to what extent each alarmin contributes to the trafficking of diverse types of cells in vivo under distinct conditions. By further understanding the role alarmins play and the mechanisms involved, ways of targeting alarmininduced trafficking of immune cells may be identified and this provide a means for regulating immune responses and treating inflammatory and autoimmune disorders.

Acknowledgment: This project has been funded in whole or in part with federal funds from the National Cancer Institute, National Institutes of Health, under contract HHSN261200800001E. The content of this publication does not necessarily reflect the views or policies of the Department of Health and Human Services, nor does mention of trade names, commercial products, or organizations imply endorsement by the U.S. Government. This Research was supported in part by the Intramural Research Program of the $\mathrm{NIH}$, National Cancer Institute. This work was also supported in part by a 973 grant (2012CB932503) from the National Key Basic Research Program of China.

Conflict of interest: The authors declare no financial or commercial conflict of interest.

\section{References}

1 Baggiolini, M. et al., Nature 1998. 392: 565568.

2 Germain, R. N. et al., Science 2012. 336: 16761681.

3 Springer, T. A. et al., Nature 1990. 346: 425434.

4 Allen, S. J. et al., Annu. Rev. Immunol. 2007. 25: $787-820$.

5 Ley, K. et al., Nat. Rev. Immunol. 2007. 7: 678689.

6 Ridley, A. J. et al., Science 2003. 302: 17041709.

7 Murphy, P. M. et al., Annu. Rev. Immunol. 1994. 12: 593-633.
8 Roussos, E. T. et al., Nat. Rev. Cancer 2011. 11: 573-587.

9 Oppenheim, J. J. et al., Curr. Opin. Immunol. 2005. 17: 359-365.

10 Bianchi, M. E. et al., J. Leukoc. Biol. 2007. 81: 1-5.

11 Lotze, M. T. et al., Nat. Rev. Immunol. 2005. 5: 331-342.

12 Seong, S. Y. et al., Nat. Rev. Immunol. 2004. 4: 469-478.

13 Yang, D. et al., Arthritis Rheum. 2004. 50: 3401-3403.

14 Yang, D. et al., Trends Immunol. 2009. 30: 531537.

15 Chertov, O. et al., J. Biol. Chem. 1996. 271: 2935-2940.

16 Territo, M. C. et al., J. Clin. Invest. 1989. 84: 2017-2020.

17 Yang, D. et al., J. Leukoc. Biol. 2000. 68: 9-14.

18 Yang, D. et al., Science 1999. 286: 525-528.

19 Biragyn, A. et al., J. Immunol. 2001. 167: 66446653.

20 Rohrl, J. et al., J. Biol. Chem. 2008. 283: 5414 5419.

21 Grigat, J. et al., J. Immunol. 2007. 179: 39583965.

22 Niyonsaba, F. et al., Int. Immunol. 2002. 14: 421-426.

23 Rohrl, J. et al., J. Biol. Chem. 2010. 285: 70287034.

24 Tyrrell, C. et al., Mol. Immunol. 2010. 47: 13781382.

25 Ito, T. et al., Tohoku J. Exp. Med. 2012. 227: 39-48.

26 Rohrl, J. et al., J. Immunol. 2010. 184: 66886694.

27 Jin, G. et al., PLoS One 2010. 5: e10993.

28 Mackenzie-Dyck, S. et al., Vet. Immunol. Immunopathol. 2011. 143: 87-107.

29 Cuesta, A. et al., Mol. Immunol. 2011. 48: 14321438.

30 Huang, H. J. et al., J. Leukoc. Biol. 1997. 61: 624-629.

31 Verbanac, D. et al., FEBS Lett. 1993. 371: 255258.

32 Yang, D. et al., J. Exp. Med. 2000. 192: 10691074.

33 Niyonsaba, F. et al., Immunology 2002. 106: 20-26.

34 Subramanian, H. et al., J. Biol. Chem. 2011. 286: 44739-44749.

35 Koczulla, R. et al., J. Clin. Invest. 2003. 111: 1665-1672.

36 Coffelt, S. B. et al., Proc. Natl. Acad. Sci. USA 2009. 106: 3806-3811.

37 Tokumaru, S. et al., J. Immunol. 2005. 175: 4662-4668.

38 Chen, K. et al., J. Immunol. 2010. 184: 33313335.

39 Deng, A. et al., J. Immunol. 2005. 174: 52435248 .

40 Yang, D. et al., Blood 2003. 102: 3396-3403.

41 Gastpar, R. et al., J. Immunol. 2004. 172: $972-$ 980.
42 Tewary, P. et al., Blood 2010. 116: 3465-3474.

43 Ryckman, C. et al., J. Immunol. 2003. 170: 3233-3242.

44 Yan, W. X. et al., J. Biol. Chem. 2008. 283: 13035-13043.

45 de la Rosa, G. et al., J. Immunol. 2008. 180 6868-6876.

46 Wolf, R. et al., J. Immunol. 2008. 181: 14991506.

47 Cornish, C. J. et al., J. Cell. Physiol. 1996. 166: 427-437.

48 Lackmann, M. et al., J. Immunol. 1993. 150: 2981-2991.

49 Yang, D. et al., J. Exp. Med. 2012. 209: 157-171.

50 Berthelot, F. et al., J. Innate. Immun. 2012. 4: 41-58.

51 Rouhiainen, A. et al., Blood 2004. 104: 1174 1182.

52 Yang, D. et al., J. Leukoc. Biol. 2007. 81: 59-66.

53 Palumbo, R. et al., J. Cell. Biol. 2004. 164: 441449.

54 Meng, E. et al., Stem Cells Dev. 2008. 17: 805813.

55 Lotfi, R. et al., Eur. J. Immunol. 2011. 41: 2021 2028.

56 Chavakis, E. et al., Circ. Res. 2007. 100: 204 212.

57 Degryse, B. et al., J. Cell Biol. 2001. 152: $1197-$ 1206.

58 Yang, J. et al., Mol. Biol. Rep. 2012. 39: 33613367.

59 Straino, S. et al., J. Invest. Dermatol. 2008. 128: 1545-1553.

60 Tang, C. H. et al., Cancer Lett. 2012. 322: 98 106.

61 Hori, O. et al., J. Biol. Chem. 1995. 270: 2575225761.

62 Huttunen, H. J. et al., J. Biol. Chem. 1999. 274: 19919-19924.

63 Komai-Koma, M. et al., Eur. J. Immunol. 2007. 37: 2779-2786.

64 Verri, W. A., Jr. et al., Ann. Rheum. Dis. 2010. 69: 1697-1703.

65 Kobayashi, T. et al., J. Immunol. 2010. 184: 6350-6358.

66 Corriden, R. et al., Purinergic. Signal. 2012. 8: 587-598.

67 Kurosaka, K. et al., J. Immunol. 2005. 174: 6257-6265.

68 Schiraldi, M. et al., J. Exp. Med. 2012. 209: 551563.

69 Wu, Z. et al., Proc. Natl. Acad. Sci. USA 2003. 100: 8880-8885.

70 Conejo-Garcia, J. R. et al., Nat. Med. 2004. 10: 950-958.

71 Vongsa, R. A. et al., J. Biol. Chem. 2009. 284: 10034-10045.

72 Orlova, V. V. et al., EMBO J. 2007. 26: 11291139.

73 Bassi, R. et al., J. Neurooncol. 2008. 87: 23-33.

74 Alves-Filho, J. C. et al., Nat. Med. 2010. 16: 708-712.

75 Niyonsaba, F. et al., J. Invest. Dermatol. 2007. 127: 594-604. 
76 Girnita, A. et al., Oncogene 2012. 31: 352-365

77 Mitola, S. et al., J. Immunol. 2006. 176: 12-15.

78 Palumbo, R. et al., J. Cell Biol. 2007. 179: 33-40.

79 Hofmann, M. A. et al., Cell 1999. 97: 889-901.

80 Hudson, B. I. et al., J. Biol. Chem. 2008. 283. 34457-34468.

81 Vogl, T. et al., Nat. Med. 2007. 13: 1042-1049.

82 van Wetering, S. et al., J. Leukoc. Biol. 1997 62: 217-226.

$83 \mathrm{Ju}$, Y. et al., Int. J. Mol. Med. 2008. 22: 657-662.

84 Chen, T. et al., J. Immunol. 2009. 182: 14491459.

85 Yang, D. et al., J. Immunol. 2004. 173: 6134 6142.

86 Andersson, U. et al., J. Exp. Med. 2000. 192 565-570.

87 Messmer, D. et al., J. Immunol. 2004. 173: 307313.

88 Sunahori, K. et al., Arthritis Res. Ther. 2006. 8: R69.

89 Kew, R. R. et al., J. Immunol. 2012. 188: 23802386

90 Pfosser, A. et al., Stem Cells 2010. 28: 376-385.

91 Ghannam, S. et al., J. Immunol. 2011. 186: 1411-1420.

92 Tjabringa, G. S. et al., J. Immunol. 2003. 171: 6690-6696.

93 Lande, R. et al., Nature 2007. 449: 564-569.

94 Gregorio, J. et al., J. Exp. Med. 2010. 207: 29212930.

95 Banchereau, J. et al., Nature 1998. 392: 245251.
96 Randolph, G. J. et al., Nat. Rev. Immunol. 2005. 5: 617-628.

97 Ginhoux, F. et al., J. Exp. Med. 2007. 204: 31333146.

98 Yang, D. et al., Annu. Rev. Immunol. 2004. 22: 181-315.

99 Campana, L. et al., J. Leukoc. Biol. 2009. 86: 609-615.

100 Manfredi, A. A. et al., J. Immunol. 2008. 180: 2270-2275.

101 Rovere-Querini, P. et al., EMBO Rep. 2004. 5: 825-830.

102 Loser, K. et al., Nat. Med. 2010. 16: 713-717.

103 Shi, Y. et al., Nature 2003. 425: 516-521.

104 Kool, M. et al., Immunity 2011. 34: 527-540.

105 Chen, G. Y. et al., Science 2009. 323: 17221725.

106 Yang, D. et al., J. Exp. Med. 2008. 205: 79-90.

107 Andersson, U. et al., Annu. Rev. Immunol. 2011. 29: 139-162.

108 Huang, Y. et al., Am. J. Transplant. 2007. 7: 799-808.

Correspondence: Dr. Joost J. Oppenheim, Laboratory of Molecular Immunoregulation, Cancer and Inflammation Program, National Cancer Institute, Frederick National

Laboratories for Cancer Research, Frederick, MD 21701, USA

Fax: +1-301-846-7042

e-mail: oppenhei@ncifcrf.gov
Additional correspondence: Dr. De Yang

e-mail: yangd@mail.nih.gov

Received: 9/11/2012

Revised: 13/3/2013

Accepted: 22/4/2013

Keywords: Adhesion - Alarmins - Cell migration $\cdot$ Chemokines $\cdot$ DCs

Abbreviations: CRAMP: cathelicidin-related antimicrobial peptide . DAMP: damageassociated molecular pattern . EDN: eosinophil-derived neurotoxin . EPC: endothelial progenitor cell . GiPCR: Goi proteincoupled receptor . HMGB1: high-mobility group box-1 protein . HMGN1: high-mobility group nucleosome-binding protein 1 . MC: mast cell - PI3K: phosphatidylinositol-3 kinase - RAGE: receptor for glycation end products SMC: smooth muscle cell

See accompanying Viewpoint:

http://dx.doi.org/10.1002/eji.201243179

The complete Cells in Motion Viewpoint series is available at:

http://onlinelibrary.wiley.com/doi/10. 1002/eji.v43.6/issuetoc

\title{
CCRL2, a fringe member of the atypical chemoattractant receptor family
}

\author{
${ }^{1}$ Department of Molecular and Translational Medicine, University of Brescia, Brescia, \\ Italy \\ ${ }^{2}$ Humanitas Clinical and Research Center, Rozzano, Italy \\ ${ }^{3}$ Department of Medical Biotechnologies and Translational Medicine, University of Milan, \\ Rozzano, Italy
}

Annalisa Del Prete ${ }^{1,2}$, Raffaella Bonecchi ${ }^{2,3}$, Annunciata Vecchi ${ }^{2}$, Alberto Mantovani ${ }^{2,3}$ and Silvano Sozzani ${ }^{1,2}$

DOI: 10.1002/eji.201243179

The term atypical chemoattractant receptors is generally used to refer to a subset of G-protein-coupled receptors devoid of chemotactic activity and characterized by the ability to scavenge chemotactic factors from the inflammatory milieu. However, emerging evidence suggests that this class of receptors is heterogeneous in function. In this Viewpoint, we discuss the properties of CCRL2, a molecule devoid of ligand scavenging functions and suggested to regulate leukocyte recruitment by alternative mechanisms.

Chemotactic receptors and their cognate ligands are key players in leukocyte tissue localization during homeostatic and inflammatory conditions. Chemotactic receptors belong to the large family of seven transmembrane G-proteincoupled receptors (GPCRs) and classically signal through the activation of phospholipase C, phosphaditylinositol-3 kinase, and MAPKs upon ligand binding $[1,2]$. Lipids, such as leukotrienes and platelet-activating factor, pathogenassociated molecular patterns (e.g. formylated peptides), and proteins may act as chemotactic agonists, with chemokines representing the major family of chemotactic proteins. Atypical chemoattractant receptors represent a small subset of GPCRs characterized by a high degree of homology with chemotactic receptors and the lack of a highly conserved sequence in the third intracellular loop, known as the DRYLAIV motif, crucial for G-protein signaling [3, 4]. Atypical chemoattractant receptors include Duffy 


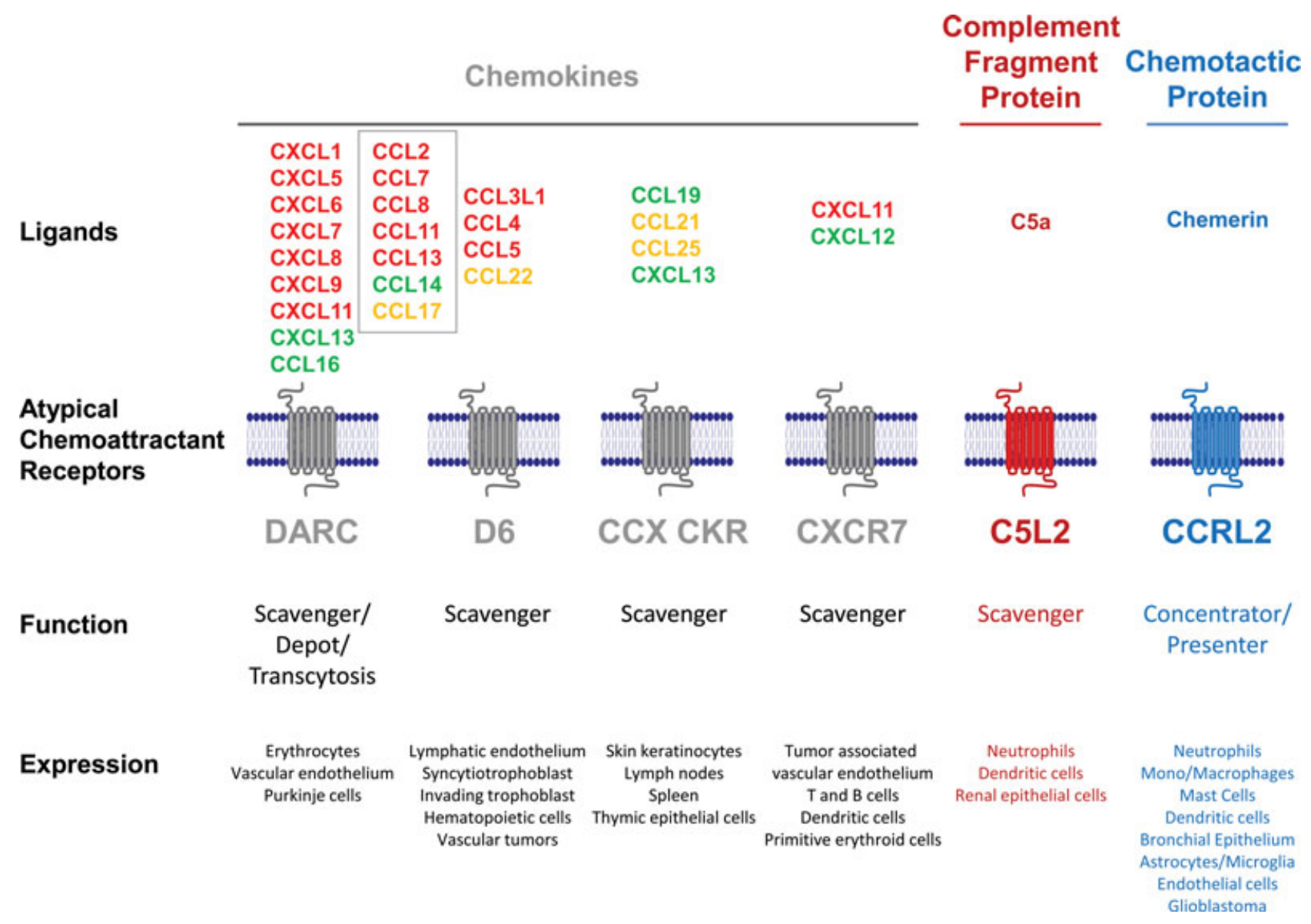

Figure 1. Chemoattractant atypical receptor ligand specificity, distribution, and function. DARC (Duffy Antigen Receptor for Chemokines) and D6 are mainly expressed on the endothelium (lymphatic and vascular, respectively); DARC is also expressed by erythrocytes, and evidence for D6 expression by leukocytes has also been provided. CCX-CKR is expressed by various tissues. CXCR7 is expressed by lymphocytes, DCs, and by tumor-associated vascular endothelium. C5L2 is expressed by neutrophils, DCs, and renal epithelial cells. CCRL2 is mostly expressed by many hematopoietic and nonhematopoietic cell types. Chemotactic factors are color-coded as proinflammatory (red), homeostatic (green), and those with mixed function (yellow). The ligands shared by DARC and D6 are in the box.

antigen/receptor for chemokines (DARC), D6, CCX Chemokine Receptor (CXXCKR), CXC chemokine receptor type 7(CXCR7), C5aR-like receptor 2 (C5L2), and CC chemokine receptor-like 2 (CCRL2) (Fig. 1). These atypical receptors all bind CC and CXC chemokines, albeit with variable degrees of promiscuity, with the exception of C5L2, which binds the chemotactic complement fragment $\mathrm{C} 5 \mathrm{a}$ and CCRL2, which binds a protein called chemerin. All these receptors are characterized by similar/common structural features, i.e. the lack of classical GPCR signaling and of chemotactic functions. All atypical chemoattractant receptors, but CCRL2, are characterized by ligand scavenging functions due to their constitutive or ligand-induced internalization and many studies have reported that these receptors play a relevant role in the control of inflammation in vitro and in vivo $[3,4]$.

\section{CCRL2 is an atypical chemoattractant receptor}

CCRL2 was cloned as an orphan receptor and is known as human chemokine receptor (HCR), because it exhibits high homology to CC chemokine receptors (about $40 \%$ amino acid identity with CCR1, CCR2, CCR3, and CCR5) [5]. The CCRL2 gene is located at the edge of the main chemokine receptor cluster in the 3p21-23 region of the genome where XCR1, CCR1, CCR3, CCR2, and CCR5 are located. Further proof that CCRL2 belongs to the family of chemokine receptors was provided by phylogenetic analysis that placed CCRL2 on a branch related to CC chemokine receptors [6]. Two transcript variants of CCRL2 derived from alternative splicing have been described and named CCRL2A and CCRL2B. The deduced protein sequences for these variants differ only by the presence of 12 additional amino acids at the
$\mathrm{N}$-terminus in CCRL2A. The CCRL2 murine ortholog, originally named LPS-inducible CC chemokine receptor (L-CCR), or E01, was originally described as an inducible gene in the mouse macrophage cell line, RAW264 [7]. In contrast to human CCRL2, mouse CCRL2 is present as a single variant with 51\% sequence identity with CCRL2B. Analysis of the CCRL2 amino acid sequence reveals the presence of two main alterations in regions that are critical for G-protein coupling and signaling. First, the substitution of an aspartic acid residue, present in almost all GPCR second transmembrane domains, for asparagine (N82), and second, an alteration of the DRYLAIV motif in the third intracellular loop (QRYLVFL); these amino acid changes are conserved among species.

Several efforts have been made to identify CCRL2 ligands. CCRL2 was 
initially described to promote chemotaxis and calcium fluxes in response to CCL2, CCL5, CCL7, and CCL8 [8] or to bind CCL19 in the absence of signaling [9]. However, these results remain controversial since other researchers have not detected any CCRL2 functional activation by these chemokines, or by many other lipid, or protein chemotactic agonists [10, 11]. More recently, CCRL2 was reported to bind, in the absence of any detectable signaling, chemerin [11], a chemotactic protein previously described as the agonist of two GPCRs, namely ChemR23/CMKLR1 and GPR1 $[12,13]$. Constitutive CCRL2 internalization has been reported by Leick et al. [9], but this result was challenged by other groups using antibody-feeding experiments in transfected CHO-K1 or L1.2 cells (Otero and Sozzani, unpublished), or chemerin-binding assays in transfected cells and primary endothelial cells $[11,14]$. Taken together, we believe that the data available thus far indicate that CCRL2 is an "atypical" chemoattractant receptor devoid of ligand scavenging properties.

\section{Regulation of CCRL2 expression}

CCRL2 expression has been detected in many lymphoid organs (spleen, lymph node, fetal liver, bone marrow) as well as in nonlymphoid organs (heart and lung) [5] (Table 1). Within the hematopoietic compartment, CCRL2 mRNA and protein have been detected in neutrophils, monocytes, dendritic cells (DCs), mast cells, NK cells, T cells, and CD34-positive cells $[11,15,16]$. In general, CCRL2 is upregulated in activated cells. Indeed, CCRL2 mRNA was initially identified as an early inducible gene in LPS-stimulated mouse macrophages [7]. Similarly, LPS alone or in combination with IFN- $\gamma$ rapidly induced CCRL2 expression in human monocytes $[15,16]$. CCRL2 is also rapidly induced in human monocyte-derived DCs [15] and in mouse bone marrow-derived DCs stimulated with LPS, Poly (I:C) or soluble CD40L with kinetics that preceded the expression of CCR7, the hallmark of lymph node migrating mature DCs [10, 17]. Mouse mast cells express CCRL2 in a constitutive manner and this expression is further upregulated over time in culture [11]. In human neutrophils, proinflammatory signals, such as LPS or TNF alone or in combination with IFN- $\gamma$ or GM-CSF, induce the upregulation of CCRL2 mRNA expression [18]; CCR2 mRNA has been found to be overexpressed in neutrophils isolated from the synovial fluid of rheumatoid arthritis patients [19]. The expression of CCRL2 in peripheral blood lymphocytes was undetectable in Patel's study [16], while $\mathrm{CD}^{+}$and $\mathrm{CD}^{+} \mathrm{T}$ lymphocytes, including most memory and a portion of naive cells, have been reported to be CCRL2-positive, especially after CD3 and IL-2 stimulation [15]. More recently, the expression of the CCRL2B splice variant in $\mathrm{B}$ cells has been found to be dependent on the cell maturation stage, with the high- est expression being observed in pro- and pre-B cells [20].

In nonhematopoietic cells, CCRL2 expression has been described in both astrocytes and microglia and expression was strongly enhanced by in vitro and in vivo stimulation with LPS [21]. In a model of experimental autoimmune encephalomyelitis, CCRL2 mRNA has been detected both in the brain and in the spinal cord of animals displaying clinical signs of the disease, predominantly being expressed by astrocytes, microglia, and infiltrating macrophages [22]. Finally, constitutive CCRL2 RNA and protein expression has been detected in barrier cells such as mouse bronchial epithelium [23] and mouse and human endothelial cells and is upregulated during inflammatory conditions (i.e. LPS, TNF- $\alpha$, IFN- $\gamma$ ) [14]. Therefore, CCRL2 is widely expressed by many hematopoietic and nonhematopoietic cell types with comparable kinetics and, generally, its expression is upregulated during inflammatory conditions.

\section{CCRL2 as a regulator of immune responses}

The current understanding of the biological functions of CCRL2 is mostly based on the use of CCRL2-deficient (CCRL2 KO) mice. Such mice are fertile, present a normal lifespan, and do not show any overt phenotype under steady-state conditions $[10,11]$; however, CCRL2 KO mice do not

Table 1. Expression and function of CCRL2 ${ }^{a)}$

\begin{tabular}{|c|c|c|c|c|}
\hline Cell type & Species & Expression & Function & References \\
\hline Neutrophils & Human & mRNA, protein & Migration? & {$[15,16,18]$} \\
\hline \multirow[t]{2}{*}{ Mono/macrophages } & Human & mRNA, protein & Unknown & {$[15,16]$} \\
\hline & Mouse & mRNA & Control of bacterial infection? & 10 \\
\hline $\mathrm{T}$ cells & Human & mRNA, protein & DC-T cell interaction? & [15] \\
\hline Mast Cells & Mouse & protein & Concentrator/Presenter & {$[11]$} \\
\hline $\mathrm{CD} 34^{+}$cells & Human & mRNA, protein & Homing? & {$[15]$} \\
\hline Pre-B cells & Human & mRNA, protein & Modulator of immune response? & {$[20]$} \\
\hline B-cell CLL & Human & protein & CCL19 internalization & {$[9]$} \\
\hline \multirow[t]{2}{*}{ Dendritic cells } & Human & mRNA, protein & DC-T-cell interaction? & [15] \\
\hline & Mouse & mRNA, protein & Lung DC migration & 10 \\
\hline Bronchial epithelium & Mouse & mRNA & Leukocyte recruitment? & {$[23]$} \\
\hline Endothelial cells & Mouse & mRNA, protein & Concentrator/presenter & {$[14]$} \\
\hline Astrocytes & Mouse & mRNA & Local immune regulator? & {$[21,22]$} \\
\hline Microglia & Mouse & mRNA & Local immune regulator? & {$[21,22]$} \\
\hline Glioblastoma cells & Human & mRNA & Migration & {$[26]$} \\
\hline
\end{tabular}

a) The current knowledge about CCRL2 distribution and function is listed along with the relevant references. A question mark indicates that the function has as yet not been formally proven. 
develop disease when used in an experimental model of mast cell-dependent, IgE-induced passive cutaneous anaphylaxis, i.e. when treated with DNP-specific IgE. Interestingly, a role for CCRL2 in mastcell activation was evident only when a suboptimal dose of DNP-specific IgE was used, while CCRL2 became dispensable in the presence of fully activating IgE concentrations. Although in vitro bone marrowderived mast cells from CCRL2 KO mice show chemotactic behavior, degranulation responses, cytokine secretion, and stimulation of T-cell proliferation after antigeninduced IgE/FcERI cross-linking [11] that are comparable with the responses seen in WT mice, the nonredundant role of CCRL2 expression in mast cells was formally proven by adoptive transfer experiments. Indeed, only the engraftment with cells obtained from WT animals, but not from CCRL2 KO mice, was able to promote IgE-dependent tissue inflammation and leukocyte infiltration in mast celldeficient mice [11].

In view of the reported expression of CCRL2 in the lung [23] and the crucial role played by lung DCs in the induction of airway hypersensitivity [24], CCRL2 KO mice were tested in a Th2 model of ovalbumin (OVA)-induced airway inflammation [10]. Following systemic immunization and aerosol challenge with the OVA antigen, CCRL2 KO mice presented a dramatic reduction in the total number of leukocytes and, in particular, of eosinophils and mononuclear cells in the bronchoalveolar lavage. The reduction of cell recruitment was associated with decreased levels of Th2 cytokines (i.e. IL-4 and IL-5) and of the eosinophil/Th2cell attracting chemokines CCL11 and CCL17. This decreased local Th2 response was directly correlated with the reduced migration of antigen-loaded lung DCs to mediastinal lymph nodes and to a decreased priming of antigen-specific $\mathrm{T}$ cells in the regional lymph nodes. Conversely, no significant role of CCRL2 was detected in the recruitment of blood DCs into the lung. Of note, CCRL2-deficient mice did not show any alteration when tested in a Th1 model of OVA/LPS-induced lung hypersensitivity, suggesting a peculiar role for CCRL2 in this Th2-skewed response [10].

The discovery that CCRL2 binds chemerin [11] shed new light on the possible biological function of this receptor. CCRL2 was shown to bind the $\mathrm{N}$-terminus of chemerin with high affinity, leaving the $C$-terminus of the protein available for interaction with cells expressing ChemR23, the functional chemerin receptor [11]. In addition, recent evidence has shown that CCRL2 is expressed under homeostatic and inflammatory conditions by human and mouse endothelial cells. Endothelial cells bind chemerin in the absence of ligand internalization, further support in a role of CCRL2 in shaping the chemerin chemotactic gradient in vivo [14]. Compatible with this model, CCRL2 KO mice present increased serum levels of circulating chemerin following systemic administration of inflammatory stimuli [14].

Although the role of CCRL2 in shaping the chemerin-chemotactic gradient is quite an interesting observation, it is currently uncertain whether the ability of CCRL2 to immobilize chemerin at the level of the endothelial barrier represents the only function of CCRL2 and whether it is sufficient to explain the phenotypes observed in CCRL2 KO mice. Since both mast cell- and DC-dependent phenotypes of CCRL2 have been shown in adoptive transfer experiments, it is likely that, at least in these two cell subsets, CCRL2 may play additional, as yet uncharacterized functions. Taken together, these data strongly suggest that additional roles, unrelated to chemerin binding, are associated with CCRL2 functions.

\section{Concluding remarks}

Atypical chemoattractant receptors comprise six members of the seven transmembrane domain receptor family and display high homology to "typical" chemotactic receptors. The atypical receptors lack a canonical "DRYLAIV" sequence and the ability to bind chemotactic agonists in the absence of chemotactic activity. However, increasing evidence points to divergent aspects of this subset of receptors. Some of these receptors bind chemokines (i.e. DARC, D6, CCX-CKR1), whereas CCRL2 binds an unrelated chemotactic protein (i.e. chemerin) and C5L2 binds a bioactive fragment of the complement cascade. With the exception of CCRL2, all these receptors have the ability to internalize their ligands, although with a different potential outcome. For most of them internalization results in chemokine degradation and scavenging, whereas for others, such as DARC, internalization has been demonstrated to cause transcytosis of the ligands [25]. Some of these receptors play a clear role in the control of the inflammatory processes (D6 and DARC), while CXCR7 has a clear role in development and CCRL2 seems to be involved in the control of both innate and adaptive immune responses. Taken together, the information available thus far points to an increasing level of heterogeneity among the atypical chemoattractant receptor family and further studies are warranted to better elucidate the function and role of these peculiar proteins.

Acknowledgments: This work was supported by AIRC (Associazione Italiana per la Ricerca sul Cancro) and Ministero dell'Istruzione, dell'Università e della Ricerca (M.I.U.R.), European Project IMI JU-funded project BeTheCure, contract 115142-2.

Conflict of interest: The authors declare no financial or commercial conflict of interest.

\section{References}

1 Rot, A. et al., Annu. Rev. Immunol. 2004. 22: 891-928.

2 Sanz, M. J. et al., Eur. J. Immunol. 2012. 42: 278-283.

3 Mantovani, A. et al., Nat. Rev. Immunol. 2006. 6: 907-918.

4 Graham, G. J. et al., Immunol. Lett. 2012. 145: 30-38.

5 Fan, P. et al., Biochem. Biophys. Res. Commun. 1998. 243: 264-268

6 Joost, P. et al., Genome. Biol. 2002. 3(11): RESEARCH0063.

7 Shimada, T. et al., FEBS Lett. 1998. 425: 490-494.

8 Biber, K. et al., J. Leukoc. Biol. 2003. 74: 243-251.

9 Leick, M. et al., Immunology 2010. 129: 536-546.

10 Otero, K. et al., Blood 2010. 116: 2942-2949.

11 Zabel, B. A. et al., J. Exp. Med. 2008. 205: 22072220.

12 Wittamer, V. et al., J. Biol. Chem. 2004. 279: 9956-9962.

13 Barnea, G. et al., Proc. Natl. Acad. Sci. USA 2008. 105: 64-69.

14 Monnier, J. et al., J. Immunol. 2012. 189: 956-967.

15 Migeotte, I. et al., Eur. J. Immunol. 2002. 32: 494-501.

16 Patel, L. et al., Cytokine 2001. 14: 27-36.

17 Sozzani, S., Cytokine. Growth Factor Rev. 2005. 16: 581-592.

18 Galligan, C. L. et al., Arthritis Rheum. 2004. 50: 1806-1814.

19 Auer, J. et al., Arthritis Res. Ther. 2007. 9: R94.

20 Hartmann, T. N. et al., Immunology 2008. 125: 252-262. 


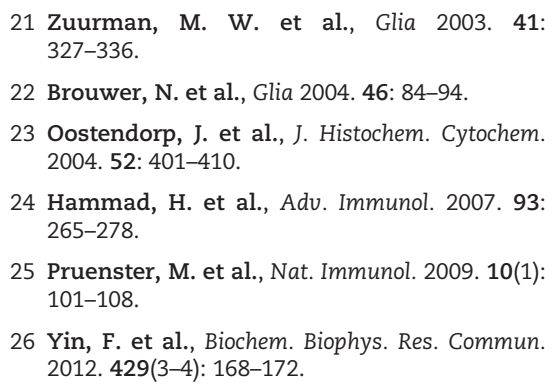

Correspondence: Prof. Silvano Sozzani, Department of Molecular and Translational Medicine, University of Brescia, Viale Europa 11, 25123 Brescia, Italy
Fax: +39-30-371-7747

e-mail: sozzani@med.unibs.it

Received: 20/11/2012

Revised: 5/3/2013

Accepted: 8/4/2013

Keywords: Cell trafficking - Cytokines • Immune regulation - Inflammation - Innate immunity

Abbreviations: CCRL2: CC chemokine receptorlike 2 - C5L2: C5aR-like receptor 2 . CXCR7:
CXC chemokine receptor type 7 . DARC: Duffy antigen/receptor for chemokines - GPCR: G-protein-coupled receptor

See accompanying Viewpoint: http://dx.doi.org/10.1002/eji.201243138

The complete Cells in Motion Viewpoint series is available at:

http://onlinelibrary.wiley.com/doi/10. 1002/eji.v43.6/issuetoc

\section{Migration and tolerance}

\section{On the road to tolerance - Generation and migration of gut regulatory $\mathrm{T}$ cells}

\section{Oliver Pabst and Günter Bernhardt}

Institute of Immunology, Hannover Medical School, Hannover, Germany
The intestinal immune system potently supports the generation of induced Treg (iTreg) cells. Within intestinal lymphoid compartments iTreg cells receive homing cues, which direct these cells to the gut lamina propria where they expand and locally suppress immune responses. Yet iTreg cells are but one side of a coin, the other side of which comprises natural Treg (nTreg) cells generated in the thymus. nTreg cells, which act in concert with iTreg cells, also acquire a diversified pattern of homing receptors. Thus iTreg and nTreg cells can enter the gut, and draining lymph nodes to cooperatively ensure intestinal homeostasis.

\section{Introduction}

The discovery that $\mathrm{T}$ cells can inhibit the proliferation and effector functions of other immune competent cells resulted in the description of a perplexing variety of repressor T cells, now subsumed under the term Treg cells. Since conventional $\mathrm{CD}^{+}$
$\mathrm{T}$ (Tconv) cells may rapidly acquire inhibitory potential in their own right after stimulation [1], a detailed functional characterization of Treg cells requires additional parameters apart from mere inhibitory capacity. Earlier work relied on CD25 as a marker for Treg cells [2] but only since the transcription factor Foxp3 was identified has it been possible to more stringently define Treg-cell subpopulations, rendering the work of different laboratories into these cells more comparable. Foxp $3^{+}$Treg cells are considered the most relevant Treg-cell subset and can be divided into those that arise in thymus or are induced in periphery from FoxP3 ${ }^{-}$ Tconv cells. For the former, the term, natural Treg (nTreg) cells was coined whereas the latter are called induced Treg (iTreg) cells.

Based on high-throughput sequencing and transcriptional profiling, recent insights demonstrated that iTreg cells and nTreg cells differ from each other, fulfilling nonredundant functions [3-6]. This makes it difficult to interpret earlier findings that engaged peripheral Treg cells as a whole as a source for experimentation. Nevertheless, a picture is emerging giving credit to the idea that nTreg cells resemble Tconv cells in their initial migratory pattern, that is, nTreg cells leaving the thymus express the homing molecules CCR7 and CD62L [7], allowing them to home to secondary lymphoid organs (SLOs) (Fig. 1). nTreg cells recirculate throughout SLOs but, in contrast to conventional $\mathrm{CD}^{+}{ }^{+} \mathrm{T}$ cells, a substantial proportion of nTreg cells shows a high tendency to propagate in the periphery even under subinflammatory conditions. This might be due to the encounter with self-antigen for which nTreg cells were initially selected for in the thymus. Such antigen-driven maturation is accompanied by down-modulation of CCR7 and CD62L and the concomitant acquisition of a distinct homing potential shaped by the peripheral SLO in which the antigen was encountered [7-9]. Unexpectedly, the TCR repertoire of nTreg cells has been shown to be as similarly diverse as that of Tconv cells [10]. In contrast, iTreg cells are recruited out of the pool of Tconv cells, and the generation of iTreg cells is particularly efficient under environmental conditions present in the intestinal immune system. Therefore, under noninflammatory conditions, iTreg cells are rare in peripheral lymphoid compartments but constitute a substantial proportion of the Treg-cell pool in the intestine. In this Viewpoint we will focus on the generation, maintenance, and function of FoxP3 ${ }^{+}$Treg cells of the intestinal system. 


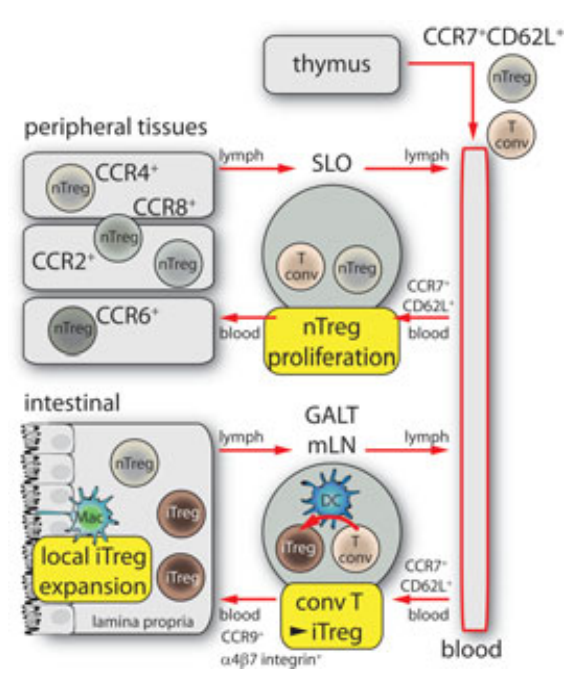

Figure 1. Treg-cell migratory routes. Tconv cells and nTreg cells leave the thymus in a naive state that enables homing to SLOs, including GALT, but not peripheral tissues. The proliferation of nTreg cells within SLOs results in a diversified nTreg-cell pool equipped with homing receptors (e.g., CCR2, CCR4, CCR6, or CCR8) that direct these cells into peripheral tissues. Activation of Tconv cells by DCs in GALT generates iTreg cells that, similar to proliferating nTreg cells, gain sitespecific homing capacity, that is, CCR9 and $\alpha 4 \beta 7$-integrin expression that direct GALTactivated cells to the gut LP (intestine). Inside the gut LP iTreg cells expand and can suppress local immune responses. nTreg and/or iTreg cells might relocate via afferent lymphatics from the gut LP to regional LNs, effectively creating a circuit of Treg-cell migratory routes.

\section{The remarkable gut}

The intestinal mucosa is permanently exposed to an exceptional load of foreign antigens; a huge amount of food constituents is resorbed from ingested food and a substantial fraction of these nutrients enters the circulation representing potential immunogens. Thus peripheral tolerance must classify these antigens accordingly to prevent deleterious immune responses such as those seen in food allergy and celiac disease. Moreover, the gut is colonized with a dense population of microbiota, including bacteria, fungi, and protozoa that possess strong immune stimulatory capacity. Handling of this hazardous mixture of antigens and microbes by the intestinal immune system involves a dedicated multilayered system of innate and adaptive mechanisms. Treg cells are but one important component of this system.
Genome-wide expression profiles revealed a typical Treg-cell signature that is partly under the control of FoxP3 and encompasses cell surface molecules, signaling components, and transcription factors differentially expressed in Treg cells compared with their expression in Tconv cells (reviewed in [11]). This Treg-cell signature is only partly recapitulated in iTreg cells arising from the converted naive Tconv cells [3], indicating that iTreg cells share some but not all aspects of nTreg cells. Similar to iTreg cells generated in vitro, the pool of Treg cells present in the intestinal lamina propria (LP) lacks aspects of the archetypical nTreg-cell signature [3], inferring that the proportion of nTreg cells is lower in the intestinal LP compared with that in peripheral lymphoid organs. This idea is also supported by TCR sequencing studies that have revealed largely overlapping TCR repertoires of thymic and peripheral lymph node (pLN) Treg cells $[10,12]$ but remarkably different TCR repertoires for Treg cells present in the intestinal LP as compared with those of pLN Treg cells [13]. Nonetheless, it is difficult to ascertain the origin of Treg cells on a single cell basis. Recently, expression of the transcription factor Helios [14] and surface molecule neuropilin-1 [15] have been suggested to be nTreg-cell markers. While the expression of neither of these markers is unique to nTreg cells, under noninflammatory conditions both are fairly nTreg-cell specific. Consistently, the gut LP of germ-free mice that do not need to establish tolerance to live gut microbiota contained a higher proportion of neuropilin-1- ${ }^{-}$Treg cells as compared with levels in colonized mice [15]. Thus, transcriptome profiles, TCR repertoire analysis, as well as analysis of neuropilin-1 expression, indicate that Treg cells in the gut are quite different compared with Treg cells at other sites, and, in particular, the gut Treg-cell population is comprised of substantial numbers of iTreg cells besides nTreg cells.

It is tempting to speculate that a higher prevalence of iTreg cells in the gut might be due to the particular intense contact with foreign antigen in that location and, in fact, Treg cells in the LP have been noted to encode TCRs directed against the intestinal microbiota [16]; however, this seemingly straightforward correlation between antigen load and iTreg-cell numbers needs to be tempered by considering the total number of Treg cells in the gut. Although Foxp $3^{+}$cells are abundant in the gut LP, they are still less frequent as compared with macrophages, plasma cells, and some other T-cell subsets. By carefully counting the number of Treg cells in longitudinal $7 \mu \mathrm{m}$ ileum cryosections for mice we observed, on average, 0.35 cells per villus (O. Pabst, unpublished observation). We expect this number might vary depending on the housing conditions and intestinal microbiota composition, as both are known to skew the Treg-cell pool in the gut $[17,18]$. In any case, the actual number of Treg cells per villus seems too limited, rendering it unlikely that the Tregcell pool with its TCR specificities might fully cover the complexity of the total antigen load. It is therefore possible that the antigen-driven generation of iTreg cells does not account for immunoregulation covering the full antigen load but might rather constitute a sophisticated pathway to deal with particularly "problematic" antigens.

\section{iTreg-cell generation in the mesenteric lymph nodes}

In vitro, TGF- $\beta$ and IL- 2 are sufficient to induce expression of Foxp3 in a substantial fraction of activated CD4 ${ }^{+} \mathrm{T}$ cells [19] and this fraction can be further increased by the addition of retinoic acid (RA) [20]. TGF- $\beta$ and RA have also been suggested to enable iTreg-cell generation following antigen administration through the oral route $[21,22]$. One commonly used experimental setup to quantify Tregcell conversion in the intestinal immune system involves the adoptive transfer of TCR-transgenic Foxp3 ${ }^{-}$T cells to recipient mice. Subsequent antigen feeding results in T-cell activation and proliferation, and the formation of a sizable number of Foxp $^{+} \mathrm{T}$ cells (Fig. 1) [3, 21, 23]. In the gut-draining mesenteric lymph nodes (mLNs), this frequency is considerably higher as compared with that of other lymphoid compartments.

Such a high capacity to generate iTreg cells could be recapitulated in vitro by stimulating Foxp3 ${ }^{-}$cells via "intestinal" DCs, that is, DCs isolated from mLNs or intestinal LP, but not those from pLNs or splenic DCs [21, 24]. Experimentally, the integrin CD103 can be used as a surrogate marker for LP-derived DCs that have migrated to the mLNs [25]. CD103 ${ }^{+}$DCs display an enhanced capacity to produce RA [26], high expression of IDO [27], thymic stromal lymphopoietin- [28] and $\beta 8$-integrin-mediated activation of TGF- $\beta$ 
[29]. Thus LP-derived DCs in the mLNs through various mechanisms support the efficient conversion of conventional T cells into iTreg cells.

Besides their ability to foster iTregcell generation, intestinal $\mathrm{CD}_{103^{+}}$DCs are imprinted with an enhanced capacity to induce the gut-homing molecules $\beta 7$-integrin and CCR9 in activated T cells $[25,26]$. Yet, in vivo induction of guthoming potential in such cells required additional factors that were provided by nonhematopoietic stroma cells [30]. We observed that BM-derived DCs fail to support gut-homing molecule induction in vitro, but can do so in vivo when injected into $\mathrm{mLN}$ afferent lymphatics. Conversely, endogenous LP-derived DCs failed to induce gut-homing molecules in lymph node grafts of peripheral origin [30]. This indicates that in vivo non-DCdependent factors contribute to the quality of the T-cell response (reviewed in [31]). We may conclude that the microenvironment of mLNs and the unique properties of intestinal DCs synergize to enable the efficient generation of iTreg cells and a guthoming signature on these cells.

\section{Shaping of the iTreg-cell pool in the LP}

Despite the previous findings regarding the generation of iTreg cells in the mLNs, such iTreg-cell generation still seems insufficient to generate intestinal tolerance. Instead, we found that tolerance to the model antigen OVA requires gut homing of iTreg cells and a subsequent local modulation of the Treg cells in the LP [23] (Fig. 1; for a recent review on oral tolerance refer to [32]). As described in "iTreg-cell generation in the mesenteric lymph nodes", gut homing requires the $\beta 7$-integrin, which binds to its ligand MadCAM-1 that is expressed by gut venules. Consistently, $\beta 7$-integrin-deficient mice fail to generate tolerance to OVA and this defect can be rescued by the adoptive transfer of $\beta 7$-competent OVA-specific T cells in WT but not MadCAM-1-deficient mice [23]. Within the gut LP, iTreg cells proliferate vigorously and macrophage-dependent signals enable a shift in the overall ratio of Foxp $^{+}$to Foxp3 ${ }^{-}$cells in favor of Treg cells. Thus the gut LP takes an active role in shaping the Treg-cell pool by expanding iTreg-cell populations, which also explains why the TCR repertoire of gut Treg cells differs from that of Treg cells of other origins. Notably, we observe Treg cells in the afferent lymph connecting the intestine to the mLNs, thus documenting that these cells can travel back to their place of birth (O. Pabst, unpublished observation). Interestingly, there is evidence that Treg-cell populations might be modulated in other tissues as well. In skin-draining LNs the frequency of skin-derived Treg cells increases after inflammation [33] and, in an allograft model, Treg-cell-mediated suppression requires the migration of Treg cells from the graft to the draining LNs [34]. Both of these settings are characterized by inflammation and differ from the subinflammatory conditions of the intestine during steady state. Still, these findings indicate that the migration of Treg cells from the gut or other peripheral tissues back into the draining LN might be a general feature of Treg-cell trafficking and have a profound role on the function of these cells. This is supported by findings suggesting that CCR7 is crucial to permit relocation of tissue-residing Treg cells to the draining LN [35].

\section{iTreg and nTreg cells: Two sides of a coin}

There are compelling data supporting an important function of iTreg cells in intestinal tolerance since oral tolerance against OVA does not require nTreg cells [22] but rather iTreg cells $[23,36]$. Thus, at least in the OVA model, iTreg cells but not nTreg cells are essential. However, it is conceivable that nTreg cells also survey the gut tissue as part of their bodywide task to protect the host from T-cell driven autoimmune responses. Beyond this surveillance role, why should not nTreg cells participate in establishing tolerance to the gut-specific antigenic load in the form of food and microbial antigens? At least in an inflammatory context, this is indeed the case. In models of experimental colitis where Treg cells need to keep immune responses to a broad heterogeneity of antigens in check, both nTreg- and iTreg-cell populations contribute in a nonredundant manner to protect from fatal disease outcomes $[4,5]$. Therefore, the local condition and the nature of the antigenic compound - ranging from food constituents and selfantigen to PAMPs - may preferentially require either iTreg or nTreg cell-borne protection and in many cases, successful Treg-cell responses might rely on the involvement of both Treg-cell subsets. Given that nTreg and iTreg cells differ in their TCR repertoire and may also diverge in the mode/efficacy of their suppressive mechanisms [6], one advantage of recruiting both cell types to participate in immune inhibition would be the availability of a combined and thus broader repertoire of TCRs, as well as broader inhibitory tools. We hypothesize that both iTreg and nTreg cells can acquire LN- and tissue-specific homing patterns upon antigen contact, even at the subinflammatory levels that characterize the daily (nondiseased) situation [8,23]. Typically, these migration patterns are not too restrictive but also permit organism-wide dissemination of Treg cells in order to communicate (and possibly coordinate) immune activities.

\section{Conclusions}

The intestine stands out with respect to the load and diversity of antigens encountered by immune cells. Along the road to fully appreciate Treg-cell contributions to intestinal homeostasis, it will be important to collect data regarding the identity of antigenic epitopes recognized by nTreg and/or iTreg cells. Moreover, the importance of recirculation between LNs and the drained extra-lymphatic tissue for the shaping and function of Treg cells deserves more attention. Treg cells might be capable to commute several times between tissue and LN (Fig. 1). This process might also allow for the body-wide dissemination of Treg-cell responses modulated in the gut.

Acknowledgments: This work was supported by Deutsche Forschungsgemeinschaft PA921/1-1 and PA921/2-1 to O.P. and BE1886/2-2 to G.B.

Conflict of interest: The authors declare no financial or commercial conflict of interest.

\section{References}

\footnotetext{
1 Tai, X. et al., Blood 2012. 119: 5155-5163.

2 Shevach, E. M., Nat. Rev. Immunol. 2002. 2: 389-400.

3 Feuerer, M. et al., Proc. Natl. Acad. Sci. USA 2010. 107: 5919-5924.

4 Haribhai, D. et al., Immunity 2011. 35 109-122.

5 Haribhai, D. et al., J. Immunol. 2009. 182 3461-3468.
} 
6 Bilate, A. M. and Lafaille, J. J., Annu. Rev. Immunol. 2012. 30: 733-758.

7 Lee, J. H. et al., J. Immunol. 2007. 178: 301-311.

8 Sather, B. D. et al., J. Exp. Med. 2007. 204: 1335-1347.

9 Fisson, S. et al., J. Exp. Med. 2003. 198: 737-746.

10 Pacholczyk, R. et al., Immunity 2006. 25: 249-259.

11 Feuerer, M. et al., Nat. Immunol. 2009. 10: 689-695.

12 Hsieh, C. S. et al., Nat. Immunol. 2006. 7: 401-410.

13 Fohse, L. et al., Eur. J. Immunol. 2011. 41: 3101-3113.

14 Thornton, A. M. et al., J. Immunol. 2010. 184: 3433-3441.

15 Weiss, J. M. et al., J. Exp. Med. 2012. 209: 1723-1742.

16 Lathrop, S. K. et al., Nature 2011. 478: 250-254.

17 Atarashi, K. et al., Science 2011. 331: 337-341.

18 Gaboriau-Routhiau, V. et al., Immunity 2009. 31: 677-689.

19 Chen, W. et al., J. Exp. Med. 2003. 198: 1875-1886.

20 Hill, J. A. et al., Immunity 2008. 29: 758-770.

21 Sun, C. M. et al., J. Exp. Med. 2007. 204: 1775-1785.
22 Mucida, D. et al., J. Clin. Invest. 2005. 115: 1923-1933.

23 Hadis, U. et al., Immunity 2011. 34: 237-246.

24 Coombes, J. L. et al., J. Exp. Med. 2007. 204: 1757-1764.

25 Johansson-Lindbom, B. et al., J. Exp. Med. 2005. 202: 1063-1073.

26 Jaensson, E. et al., J. Exp. Med. 2008. 205: 2139-2149.

27 Matteoli, G. et al., Gut 2010. 59: 595604.

28 Spadoni, I. et al., Mucosal Immunol. 2012. 5: 184-193.

29 Worthington, J. J. et al., Gastroenterology 2011. 141: 1802-1812.

30 Hammerschmidt, S. I. et al., J. Exp. Med. 2008. 205: 2483-2490.

31 Pabst, O. et al., J. Mol. Med. (Berl.) 2009. 87: 945-951.

32 Pabst, O. and Mowat, A. M., Mucosal Immunol. 2012. 5: 232-239.

33 Tomura, M. et al., J. Clin. Invest. 2010. 120: 883-893.

34 Zhang, N. et al., Immunity 2009. 30: 458-469.

35 Menning, A. et al., Eur. J. Immunol. 2007. 37: 1575-1583.

36 Curotto de Lafaille, M. A. et al., Immunity 2008. 29: 114-126.
Correspondence: Dr. Oliver Pabst, Institute of Immunology, Hannover Medical School, Carl-Neuberg Straße 1, 30625 Hannover, Germany

e-mail: Pabst.Oliver@MH-Hannover.de

Fax: +49-511-5329722

Received: 15/11/2012

Revised: 5/3/2013

Accepted: 21/3/2013

Keywords: Cell migration - Intestinal immunity $\cdot$ Mucosal immunity $\cdot$ Tolerance

Abbreviations: iTreg: induced Treg · LP: lamina propria - mLN: mesenteric lymph node . nTreg: natural Treg · pLN: peripheral lymph node - RA: retinoic acid - SLO: secondary lymphoid organ - Tconv: conventional

See accompanying Viewpoint: http://dx.doi.org/10.1002/eji.201243192

The complete Cells in Motion Viewpoint series is available at:

http://onlinelibrary.wiley.com/doi/10. 1002/eji.v43.6/issuetoc

\title{
Thymus-homing dendritic cells in central tolerance
}

\author{
Husein Hadeiba ${ }^{1,2}$ and Eugene C. Butcher ${ }^{1,2,3}$ \\ ${ }^{1}$ Laboratory of Immunology and Vascular Biology, Department of Pathology, Stanford \\ University School of Medicine, Stanford, CA, USA \\ ${ }^{2}$ Palo Alto Institute for Research and Education, Palo Alto, CA, USA \\ 3 The Center for Molecular Biology and Medicine, Veterans Affairs Palo Alto Health Care \\ System, Palo Alto, CA, USA
}

DOI: $10.1002 /$ eji.201243192

Central tolerance is critical in establishing a peripheral $\mathrm{T}$-cell repertoire purged of functional autoreactive $\mathrm{T}$ cells. One of the major requirements for effective central tolerance is the presentation of self and other innocuous antigens (Ags), including food, gut flora, or airway allergens, to developing $\mathrm{T}$ cells in the thymus. This seemingly challenging task can be mediated in some cases by ectopic expression of tissue-specific Ags by thymic epithelial cells or by entry of systemic blood-borne Ags into the thymus. More recently, thymic hom- ing peripheral dendritic cells (DCs) have been proposed as cellular transporters of peripheral tissue-specific Ags or foreign innocuous Ags. The aim of this viewpoint is to discuss the three principal thymic DC populations and their trafficking properties in the context of central tolerance. We will first discuss the importance of peripheral DC trafficking to the thymus and then compare and contrast the three DC subsets. We will describe how they were characterized, describe their trafficking to and their microenvironmental positioning in the thymus, and discuss the functional consequence of thymic trafficking and localization on thymic selection events.

Central tolerance is the mechanism by which newly developing T cells as well as $\mathrm{B}$ cells are rendered nonreactive to selfantigens (Ags). In order for central tolerance of $\mathrm{T}$ cells to occur, self-Ags, including tissue-specific Ags (TSAs), must be available in sufficient concentrations in the thymus to be processed and presented on MHC molecules to developing thymocytes. Early studies have shown that blood-borne Ags can access the thymic medulla and 
induce clonal deletion mediated by thymic dendritic cells (DCs) [1-3]. It was therefore proposed that developing thymocytes are censored mainly to systemic peripheral Ags that can access the thymus via the blood stream, but many potentially autoreactive T-cell clones specific to compartmentalized TSAs would ignore their cognate $\mathrm{Ag}$ in the periphery via "clonal ignorance" $[4,5]$. However, the discovery of promiscuous gene expression by specialized thymic epithelial cells (TECs) in later studies and the identification of the nuclear transcription factor AIRE have broadened our understanding of how the thymus projects the entire "peripheral self" to developing thymocytes [6]. In these exciting studies, many TSAs were found to be expressed by medullary TECs (mTECs) under the control of AIRE [7-9], although AIRE-independent mechanisms of promiscuous TSA expression by mTECs have been reported [10]. In addition to mTECs, bone marrow (BM) derived thymic DCs were also shown to play a major role in presenting ectopically expressed TSA to developing thymocytes $[11,12]$. Therefore, the relationship between thymic DCs and mTECs in the induction of central tolerance has been addressed in several studies where mTECs can either (i) act as an $\mathrm{Ag}$ pool for cross-presenting thymic DCs [11] and/or (ii) autonomously present Ags directly to developing $\mathrm{T}$ cells via macroautophagy $[13,14]$. However, the relative contributions of thymic DCs and mTECs to central tolerance induction are still not clear. In some studies, mTECs have been shown to be more efficient in selecting regulatory $\mathrm{T}$ (Treg) cells, whereas thymic DCs supported deletion $[15,16]$. In contrast, BM-derived APCs were also shown to generate Treg cells but under rather artificial experimental conditions [17-19]. More importantly though, ectopic gene expression in the thymus might not efficiently present temporally regulated gene products, or the plethora of Ags of the gut microbiome, food Ags, or other foreign but innocuous Ags that access mucosal sites [6]. Therefore, alternative mechanisms must exist to broaden the spectrum of TSAs or innocuous Ags presented to developing $\mathrm{T}$ cells.

The role of extrathymic DCs in peripheral tolerance is well established: immature DCs or DCs matured in the absence of pathogen pattern recognition signals can efficiently induce adaptiveTreg cells in peripheral lymphoid tissues [20]. However, studies in the past decade suggest that peripheral migratory DC popula- tions can also transport peripheral Ags to the thymus for central tolerance [21]. Seminal studies by Bonasio et al. [21] showed that exogenous bulk DCs from the spleen can access the thymus after adoptive transfer, and that endogenous peripheral DCs also home into the thymus in parabiosis experiments. These studies also established the trafficking programs utilized by bulk splenic DCs to access the thymus, which involve P-selectin and interactions of the DC integrin $\alpha 4 \beta 1$ with its endothelial ligand VCAM-1 [21]. The multistep DC homing cascade from the blood into the thymus was found to be also dependent on a pertussis-toxin sensitive G-protein-coupled chemokine receptor [21], and subsequent studies from our group defined a key role for the chemokine receptor CCR9 in thymic recruitment of plasmacytoid DCs (pDCs) [22]. Chemokines and their receptors also regulate the homing of DC subsets within the thymus, directing localization to the medulla, corticomedullary (CM) junction, and/or perivascular spaces $[23,24]$.

The functional consequences of DC trafficking and microenvironmental homing in the thymus have begun to be elucidated (reviewed in [12, 25, 26]), with evidence that DCs can transport peripheral Ags to the thymus to mediate clonal deletion of developing Ag-specific thymocytes, and the DCs may also direct the selection of natural Treg (nTreg) cells depending on the nature of the DC population $[26,27]$. Thymic DCs are heterogenous and not all subsets are migratory $[28,29]$. Up to $50 \%$ of thymic DCs appear to arrive from the peripheral circulation [30], potentially bearing peripheral tissue Ags for thymocyte selection. Three subtypes of thymic DCs have been characterized in the mouse: CD11 $c^{\text {low }}$ B220 ${ }^{+}$pDCs and two phenotypically and functionally distinct subsets of $\mathrm{CD} 11 \mathrm{c}^{+}$ B220- conventional DCs (cDCs) [31]. The thymic $\mathrm{cDC}$ populations have been described as CD8- $\alpha^{\text {low }}$ CD11 $b^{+}$SIRP- $\alpha^{+}$ cDCs and CD8- $\alpha^{\text {high }}$ CD11b $^{-}$SIRP- $\alpha^{-}$ cDCs, which we will denote in this viewpoint as SIRP- $\alpha^{+}$and SIRP- $\alpha^{-}$cDCs, respectively [31]. Similar thymic DC populations have been described in the human [32]. Table 1 summarizes the thymic DC populations and their role in central tolerance.

\section{Migratory (thymus homing) SIRP- $\alpha^{+}$ cDCs}

Thymic DCs were originally thought to arise intrathymically from a common
DC/T-cell precursor [33]. Experiments performed a decade later, using selective combinations of normal, parabiotic, and radioablated mice revealed distinct origins of $\mathrm{CD} 8-\alpha^{+}$versus $\mathrm{CD} 8-\alpha^{-}$cDC subsets in the adult mouse thymus (SIRP- $\alpha$ expression was not monitored on DCs in these earlier studies) [30]. Subsequent studies, using adoptive transfer experiments, parabiosis, and fetal thymic transplantation, established that the SIRP- $\alpha^{+}$ $\left(\mathrm{CD} 8-\alpha^{-}\right) \mathrm{cDC}$ population migrates into the thymus from the blood [26, 31], whereas the SIRP- $\alpha^{-}\left(\mathrm{CD} 8-\alpha^{+}\right)$subset develops intrathymically from BM-derived precursors (see section "Thymus resident (nonmigratory) SIRP- $\alpha^{-} c D C s$ " below). In coculture with thymocytes, SIRP- $\alpha^{+}$cDCs induced clonal deletion of Ag-specific T cells, and also the development of thymic Treg cells [27]. In fact, to date SIRP- $\alpha^{+}$ cDCs are the only thymic DC population that has been shown to induce nTreg cells in vitro from developing thymocytes. Even though studies on SIRP- $\alpha^{+}$cDCs have focused mainly on a model of $\mathrm{CD}^{+}{ }^{+} \mathrm{T}$-cell deletion [23], the poor-to-modest crosspresentation ability of SIRP- $\alpha^{+}$cDCs [29] would suggest that they do not play a major role in the thymic deletion of $\mathrm{CD}^{+}$ $\mathrm{T}$ cells.

Unlike the bulk of thymic DCs, which are found in the medulla and are sparsely detectable in the cortex [34-36], SIRP- $\alpha^{+}$ cDCs are found primarily in the cortex and the perivascular regions of the thymus [23]. They are highly endocytic and have been shown to sample blood-borne Ags [23]. SIRP- $\alpha^{+}$cDCs, but not SIRP- $\alpha^{-}$ DCs or pDCs, were shown to be selectively decreased in the thymus of CCR2-deficient mice, suggesting a role for CCR2 and its ligands in their development, homing, or survival [23]. Moreover, consistent with a role for SIRP- $\alpha^{+}$cDCs in thymic selection, CCR2-deficient mice exhibited a significant if modest impairment in the ability of i.v. injected Ags to induce clonal deletion of Ag-specific thymocytes [23]. BM SIRP- $\alpha^{+}$cDCs have been shown to egress into peripheral blood in response to CCR2mediated signals, which might explain the deficiency in the thymus, but monocyte chemotactic protein-2 (MCP-2 or CCL8), a potential ligand for CCR2 [37], was constitutively detected in the thymic perivascular region where the SIPR- $\alpha^{+}$cDCs are localized [23]. Moreover, CCR2 was expressed by a portion of intrathymic SIRP$\alpha^{+}$cDCs, but not SIRP- $\alpha^{-}$cDCs [23]. Thus, although direct experimental confirmation is needed, it is likely that CCR2-CCL8 
Table 1. Thymic DC populations.

\begin{tabular}{|c|c|c|c|c|c|}
\hline $\begin{array}{l}\text { Thymic DC } \\
\text { population }\end{array}$ & $\begin{array}{l}\text { DC } \\
\text { phenotype }{ }^{a)}\end{array}$ & $\begin{array}{l}\text { DC chemokine } \\
\text { receptor }\end{array}$ & $\begin{array}{l}\text { Thymic } \\
\text { chemokine ligand }\end{array}$ & $\begin{array}{l}\text { Thymic } \\
\text { localization }\end{array}$ & Central tolerance modalities ${ }^{b}$ \\
\hline pDC & $\begin{array}{l}\mathrm{CCR9}^{+} \\
\mathrm{PDCA}^{-} 1^{+} \\
{\mathrm{B} 220^{+}} \\
\text {CD11clow }\end{array}$ & CCR9 [22] & CCL25 [61] & CM [22] & $\begin{array}{l}\text { Clonal deletion }[22,26,27] \\
\text { nTreg induction (human) [55] }\end{array}$ \\
\hline "Migratory" cDC & $\begin{array}{l}\text { SIRP- } \alpha^{+} \\
\text {CD8- } \alpha^{\text {low }} \\
\text { CD11b } \\
\text { B220 }^{+} \\
\text {CD11c }^{+}\end{array}$ & CCR2 [23] & CCL8 [23] & Cortex/PV [23] & $\begin{array}{l}\text { Clonal deletion }[23] \\
\text { nTreg induction }[26,27]\end{array}$ \\
\hline “Resident" cDC & $\begin{array}{l}\text { SIRP- } \alpha^{-} \\
\text {CD8- } \alpha^{+} \\
\text {CD11b } \\
\text { B220- } \\
\text { CD11c }^{+}\end{array}$ & XCR1 [24] & XCL1 [24] & Medulla [24] & $\begin{array}{l}\text { Clonal deletion }[26,27] \\
\text { nTreg induction }[24]\end{array}$ \\
\hline
\end{tabular}

a) The table summarizes the cell surface phenotype, known chemokine receptor expression, and thymic chemokine ligands implicated in the recruitment or microenvironmental positioning in the thymus of each thymic DC population.

b) The known consequences of the DC populations on thymic selection modalities are indicated.

nTreg: natural regulatory T cells; CM: corticomedullary region; PV: perivascular spaces.

contributes to intrathymic localization of SIRP- $\alpha^{+}$cDCs, particularly in the perivascular spaces. Taken together, the ability of SIRP- $\alpha^{+}$thymic cDCs to sample bloodborne Ags, their unique intrathymic localization, and their ability to induce clonal deletion and nTreg induction [23,27] suggest a specialized role for SIRP- $\alpha^{+}$thymic cDCs in the development of central tolerance to blood-borne Ags. Since SIRP- $\alpha^{+}$ cDCs migrate into the thymus from the blood, they may also contribute to central tolerance through the presentation of Ags that they acquire in the periphery prior to thymic localization, although this possibility remains to be assessed.

\section{Thymus resident (nonmigratory) SIRP- $\alpha^{-}$cDCs}

SIRP- $\alpha^{-}$cDCs comprise the most abundant thymic DC population: They make up approximately two-thirds of cDCs and one-half of all DCs in the thymus [2931]. SIRP- $\alpha^{-}$cDCs express CD8- $\alpha$ and, such as splenic CD8- $\alpha^{+}$cDCs, they can cross-present exogenous Ags into the MHC class I pathway for presentation to $\mathrm{CD} 8^{+}$ $\mathrm{T}$ cells. They are found predominantly in the medulla and have been shown to cross-present TSAs expressed ectopically by mTECs to developing thymocytes for central tolerance [29, 38, 39]. Unlike migratory SIRP- $\alpha^{+}$cDCs, SIRP- $\alpha^{-}$cDCs are resident cells: they arise from intrathymic precursors and do not exchange efficiently with the circulating peripheral DC pool [31]. Despite being derived from intrathymic precursors, there has been significant debate on whether thymic SIRP$\alpha^{-}$cDCs derive from a common T/DC progenitor or from separate T-cell and DCprogenitors that seed the thymus $[[30,31$, 33] versus $[40,41]$, respectively]. Recent cell lineage tracing experiments and studies using fluorescent reporter mice argue that $\mathrm{T}$ cells and myeloid cells (including thymic DCs) arise from distinct precursors in the thymus [42, 43]. Nevertheless, the intrathymic precursor of thymic SIRP- $\alpha^{-}$cDCs is derived from thymic homing BM progenitors. CCR7 and CCR9 have been implicated in thymic recruitment of BM-derived thymocyte precursors [44-46], and it is likely that one or both of these chemokine receptors are involved in controlling the representation of the SIRP- $\alpha^{-}$cDC population in the thymus as well. In fact, in mixed BM competitive chimeras where WT- and CCR9deficient BM-derived DCs develop side by side, the majority of SIRP- $\alpha^{-}$thymic cDCs are derived from WT versus CCR9-deficient progenitors, whereas WT and CCR9 ${ }^{-/-} \mathrm{BM}$ contribute equally well to the migratory SIRP- $\alpha^{+}$cDC compartment in the thymus [22]. Moreover, thymic SIRP- $\alpha^{-}$cDCs have been found to be even more significantly reduced in numbers in CCR7/CCR9-double deficient mice (our unpublished findings).
Regardless of the mechanisms of precursor recruitment, SIRP- $\alpha^{-}$cDCs are believed to represent thymic resident $\mathrm{cDCs}$ that can tap into self-Ag reservoirs that may be less accessible to the migratory DC subsets [11]. It would be interesting to assess in future studies whether incoming migratory SIRP- $\alpha^{+}$cDCs could also tap into the promiscuously expressed thymic Ag pool or whether instead migratory cDCs with potentially poor cross-presenting capabilities import only extrathymic Ags into the thymus, particularly those from mucosal sites.

Mechanisms of DC recruitment to the thymic medulla, where most thymic DCs and, in particular, the resident thymic subset reside, have recently been elucidated. The chemokine XCL1 (also known as lymphotactin) is produced predominantly by the MHC class II $^{\text {high }}$ subpopulation of mTECs and mediates medullary accumulation of thymic DCs that express the cognate XCR1 receptor [24]. In contrast, CCR7 and CXCR4 play a minor role, if any, in the accumulation of DCs in the thymic medulla. Among the thymic DC subpopulations, XCR1 was most highly expressed on lymphoid CD11b ${ }^{-}$DCs, a population that largely overlaps with resident SIRP- $\alpha^{-}$cDCs (these studies did not characterize thymic DCs based on SIRP- $\alpha$ expression). Moreover, in Xcl1-deficient mice, thymic DCs failed to localize in the medulla and instead accumulated in 
the deep cortex and CM regions [24]. XCL1-mediated accumulation of CD11bcDCs to the medulla may contribute to efficient interactions between mTECs and $\mathrm{CD}_{11 \mathrm{~b}^{-}}$cDCs. Xcl1 deficiency also resulted in reduced numbers of thymic nTreg cells [24], suggesting, albeit indirectly, that SIRP- $\alpha^{-}$, as well as SIRP$\alpha^{+}$cDCs (as discussed in the "Migratory (thymus homing) SIRP- $\alpha^{+} c D C s$ " section above), may be competent to induce nTreg cells and that their induction of nTreg cells may depend upon their medullary localization.

In sum, thymic cDCs can be divided into two major subsets: a "resident" SIRP- $\alpha^{-}$ CD8- $\alpha^{+} \mathrm{CD}_{11 b^{-}}$population and a "migratory" SIRP- $\alpha^{+}$CD8- $\alpha^{-}$CD11b ${ }^{+}$cDC population. XCL1 expressed by thymic mTECs targets the "resident" SIRP- $\alpha^{-}$cDCs to the medulla [24] where they cooperate with mTECs $[11,47]$. The interaction between such SIRP- $\alpha^{-}$cDCs and mTECs is thought to contribute to nTreg-cell induction. CCR2-CCL8 interactions, on the other hand, may be important in positioning SIRP- $\alpha^{+}$migratory cDCs to the cortex and the perivascular regions of the thymus where they can sample soluble bloodborne Ags [23], and could also potentially present Ags that they transport from the periphery.

\section{CCR9 $^{+}$pDCs and thymic transport of peripheral Ags}

pDCs are important innate immune cells that produce type I IFNs in response to viral infections [48]. Early studies showed that thymic pDCs are recruited from the blood, as opposed to originating from intrathymic precursors [31]. In their unactivated immature state, pDCs are poor presenters of Ags, leading to suggestions that a primary role of pDCs in the thymus, as elsewhere, might simply be to protect the tissue from viral infections [28]. However, in the absence of microbial stimulation, pDCs can induce immune tolerance in the periphery through the induction of Treg cells [49].

We described a tolerogenic population of pDCs in lymphoid tissues that expresses the chemokine receptor CCR9 [50], which is involved not only in homing of memory and effector lymphocyte populations to the small intestines $[51,52]$, but also in progenitor T-cell homing to the thymus [46]. CCR9 ${ }^{+}$pDCs efficiently induce Treg cells from peripheral $\mathrm{T}$ cells and inhibit immune responses in vitro and in vivo [50]. The high expression of CCR9 by these immunosuppressive pDCs, the involvement of CCR9 in T-cell progenitor recruitment to the thymus, and the known expression of its chemokine ligand CCL25 by TECs led us to the finding that CCR9 mediates peripheral pDC trafficking to the thymus [22]. Interestingly, CCR9 deficiency did not completely block thymic pDC recruitment in our studies, suggesting additional, overlapping homing mechanisms: CCR7 is a candidate, since CCR7 as well as CCR9 participates in T-cell progenitor homing into the thymus $[44,45]$, and a recent study has revealed a functional role (in LN homing) for low-level CCR7 expression on circulating pDCs [53]. In agreement with previous studies implicating P-selectin and $\alpha_{4}$ integrins in thymic DC homing $[21,54]$, we also found that pDCs expressed $\alpha_{4}$ and P-selectin ligands and endogenous thymic pDC migration was inhibited by $\alpha_{4}$ integrin blockade [22]. Thus, peripheral pDC homing to the thymus likely employs the same adhesion cascade used by T-cell progenitors and bulk splenic DCs, although pDC recruitment to the thymus appears to be more dependent on CCR9 [22, 44, 45].

Ag-loaded peripheral pDCs that access the thymus localize to the CM junction and mediate efficient clonal deletion of developing Ag-specific thymocytes [22]. In our hands, we did not observe thymic nTregcell induction after i.v. injection of $\mathrm{Ag}$ loaded peripheral pDCs [22]. This is consistent with earlier studies in which mouse thymic pDCs failed to efficiently induce Treg-cell production from thymocytes in vitro [27]. In contrast, human thymic pDCs can induce $\mathrm{FOXP}^{+}$Treg cells in culture models, and TSLPR ${ }^{+}$pDCs co-localize with FOXP3 $^{+}$Treg cells in the human thymus [55]. The failure of Treg-cell induction by thymic DCs in many mouse model studies ([21-23] and reviewed in $[12,26])$ may be a function of the transgenic TCR-Ag model systems employed, reflecting unique characteristics of signaling through the transgenic TCR. Several studies have indeed correlated TCR affinity and signal strength with differential effects on clonal deletion versus Treg-cell induction, such that highaffinity interactions mediate clonal deletion, whereas lower affinities, in comparison, rescue developing thymocytes from death and shunt their development into Treg cells [13, 56-59]. Therefore, caution has to be exercised in interpreting the results: Additional studies will be required to determine critically the importance of pDC versus "migratory" and "resident" CDC populations, and of TCR affinity, in
Treg-cell induction versus clonal deletion modalities of central tolerance.

It is interesting to consider that, in addition to peripheral self-Ags, thymic homing DCs may also transport innocuous foreign Ags to the thymus, such as those from allergens in the respiratory tract or food or innocuous flora in the digestive tract [6]. Indeed, CCR9 can mediate pDC localization to the intraepithelial compartment of the gut wall, a site well positioned to sample Ags in the gut lumen $[22,60]$. Whether peripheral DCs, in particular pDCs, can transport locally endocytosed mucosal Ags to the thymus remains to be determined, however.

Importantly, transport of peripheral Ags into the thymus needs to be carefully controlled to prevent central tolerance to pathogen-associated molecules. Such control appears to be achieved at least in part by suppression of thymic homing of peripheral DC populations by pathogen-associated pattern recognition through TLRs $[21,22]$. In the case of pDCs, we showed that TLR9 ligands for example, which mimic microbial DNA, efficiently downregulate CCR9, preventing pDC-mediated transport of pathogen-associated Ags into the thymus [22].

\section{Concluding remarks}

In conclusion, $\mathrm{CCR}^{+}$pDCs and SIRP- $\alpha^{+}$ cDCs comprise the rapidly exchanging migratory DCs in the thymus [31]. CCR9 is critical in targeting pDCs to the CM region of the thymus where they participate in clonal deletion of developing Ag-specific thymocytes [22]. Moreover, pDCs may be specialized in transporting innocuous peripheral Ags into the thymus, potentially including food or intestinal microflora Ags. Migratory SIRP $-\alpha^{+}$cDCs, however, are targeted to the thymic cortex and localize to perivascular spaces in part by means of CCR2-CCL8 interactions [23], and contribute to clonal deletion and nTreg-cell induction [27] to systemic blood-borne Ags [23]. Whether they also transport peripherally endocytosed Ags to the thymus for central tolerance, supplementing the transport function of pDCs, remains to be determined. Finally, the resident SIRP- $\alpha^{-}$ cDC population, believed by some to arise from a common T-cell/DC progenitor (reviewed in [12]), is targeted by XCR1-dependent migration to the thymic medulla [24], where these cells 
cross-present mTEC-expressed Ags to developing thymocytes (reviewed in [12]). In general, all the thymic homing DCs, and the migratory precursors of thymic resident DCs, are assumed to home from the blood into the thymus by means of common mechanisms of P-selectin rolling and $\alpha 4 \beta 1 /$ VCAM-1-mediated arrest on vessels of the CM junction [21], but the involvement of multiple chemoattractants allows differential recruitment and microenvironmental positioning of these specialized APCs that mediate central tolerance to self- and innocuous nonself-Ags.

Acknowledgements: H.H. is a recipient of an Investigator Career Award from the Arthritis Foundation and was a fellow under NIH Training Grant AI07290. Supported in part by NIH grants AI093981, DK084647, and AI047822 to E.C.B., and by a CIRM grant to H.H. and E.C.B.

Conflict of interest: The authors declare no financial or commercial conflict of interest.

\section{References}

1 Kyewski, B. A. et al., Nature 1984. 308: 196-199.

2 Kyewski, B. A. et al., J. Exp. Med. 1986. 163: 231-246.

3 Volkmann, A. et al., J. Immunol. 1997. 158: 693-706.

4 Nossal, G. J., Ann. N. Y. Acad. Sci. 1993. 690: 34-41.

5 Nossal, G. J., Cell 1994. 76: 229-239.

6 Derbinski, J. and Kyewski, B., Curr. Opin. Immunol. 2010. 22: 592-600.

7 Pitkanen, J. and Peterson, P., Genes Immun. 2003. 4: 12-21.

8 Anderson, M. S. et al., Science 2002. 298: 1395-1401.

9 Liston, A. et al., Nat. Immunol. 2003. 4: 350-354. 10 Derbinski, J. et al., J. Exp. Med. 2005. 202: 33-45.

11 Koble, C. and Kyewski, B., J. Exp. Med. 2009. 206: 1505-1513.

12 Klein, L. et al., Nat. Rev. Immunol. 2009. 9: 833-844.

13 Hinterberger, M. et al., Nat. Immunol. 2010. 11: 512-519.

14 Nedjic, J. et al., Nature 2008. 455: 396-400.

15 Apostolou, I. et al., Nat. Immunol. 2002. 3: 756-763.

16 Aschenbrenner, K. et al., Nat. Immunol. 2007. 8: $351-358$
17 Watanabe, N. et al., Nature 2005. 436: 1181-1185.

18 Spence, P. J. and Green, E. A., Proc. Natl. Acad. Sci. USA 2008. 105: 973-978.

19 Wirnsberger, G. et al., Proc. Natl. Acad. Sci. USA 2009. 106: 10278-10283.

20 Steinman, R. M. et al., Annu. Rev. Immunol. 2003. 21: 685-711.

21 Bonasio, R. et al., Nat. Immunol. 2006. 7: 1092-1100.

22 Hadeiba, H. et al., Immunity 2012. 36: 438-450.

23 Baba, T. et al., J. Immunol. 2009. 183: 3053-3063.

24 Lei, Y. et al., J. Exp. Med. 2011. 208: 383-394.

25 Goldschneider, I. and Cone, R. E., Trends Immunol. 2003. 24: 77-81.

26 Proietto, A. I. et al., Immunol. Cell Biol. 2009. 87 39-45.

27 Proietto, A. I. et al., Proc. Natl. Acad. Sci. USA 2008. 105: 19869-19874.

$28 \mathrm{Wu}, \mathrm{L}$. and Shortman, K., Semin. Immunol. 2005. 17: 304-312.

29 Proietto, A. I. et al., Immunol. Cell Biol. 2008. 86: 700-708.

30 Donskoy, E. and Goldschneider, I., J. Immunol. 2003. 170: 3514-3521.

31 Li, J. et al., J. Exp. Med. 2009. 206: 607-622.

32 Vandenabeele, S. et al., Blood 2001. 97: 1733-1741.

33 Ardavin, C. et al., Nature 1993. 362: 761-763.

34 Barclay, A. N. and Mayrhofer, G., J. Exp. Med. 1981. 153: 1666-1671.

35 Flotte, T. J. et al., Am. J. Pathol. 1983. 111 112-124.

36 Kurobe, H. et al., Immunity 2006. 24: 165-177.

37 Murphy, P. M. et al., Pharmacol. Rev. 2000. 52: 145-176.

38 Heino, M. et al., Eur. J. Immunol. 2000. 30: 1884-1893.

39 Kyewski, B. and Derbinski, J., Nat. Rev Immunol. 2004. 4: 688-698.

40 Rodewald, H. R. et al., Proc. Natl. Acad. Sci. USA 1999. 96: 15068-15073.

41 Radtke, F. et al., J. Exp. Med. 2000. 191: 1085-1094.

42 Schlenner, S. M. et al., Immunity 2010. 32: 426-436.

43 Luche, H. et al., Eur. J. Immunol. 2011. 41: 2165-2175.

44 Krueger, A. et al., Blood 2010. 115: 1906-1912.

45 Zlotoff, D. A. et al., Blood 2010. 115: 1897-1905.

46 Uehara, S. et al., J. Immunol. 2002. 168 2811-2819.

47 Gallegos, A. M. and Bevan, M. J., J. Exp. Med. 2004. 200: 1039-1049.

48 Colonna, M. et al., Nat. Immunol. 2004. 5: 1219-1226.

49 Morelli, A. E. and Thomson, A. W., Nat. Rev. Immunol. 2007. 7: 610-621.
50 Hadeiba, H. et al., Nat. Immunol. 2008. 9 1253-1260.

51 Pabst, O. et al., J. Exp. Med. 2004. 199: 411-416.

52 Kunkel, E. J. et al., J. Exp. Med. 2000. 192: 761-768.

53 Seth, S. et al., J. Immunol. 2011. 186: 3364-3372.

54 Scimone, M. L. et al., Proc. Natl. Acad. Sci. USA 2006. 103: 7006-7011.

55 Hanabuchi, S. et al., J. Immunol. 2010. 184 2999-3007.

56 Starr, T. K. et al., Annu. Rev. Immunol. 2003. 21 139-176.

57 Relland, L. M. et al., J. Immunol. 2009. 182 1341-1350.

58 Jordan, M. S. et al., Nat. Immunol. 2001. 2 301-306.

59 Atibalentja, D. F. et al., J. Immunol. 2009. 183 7909-7918.

60 Wendland, M. et al., Proc. Natl. Acad. Sci. USA 2007. 104: 6347-6352.

61 Wurbel, M. A. et al., Eur. J. Immunol. 2000. 30: 262-271.

Correspondence: Dr. Husein Hadeiba, The Center for Molecular Biology and Medicine, Veterans Affairs Palo Alto Health Care System, 3801 Miranda Avenue (154B) Building 101, Room B4-145, Palo Alto, CA 94304, USA

Fax: +1-650-858-3986

e-mail: hadeiba@stanford.edu

Additional correspondence: Dr. Eugene Butcher, The Center for Molecular Biology and Medicine, Veterans Affairs Palo Alto Health Care System, 3801 Miranda Avenue (154B), Building 101, Room B4-145, Palo Alto, CA 94304, USA

e-mail: ebutcher@stanford.edu

Received: 26/11/2012

Revised: 18/4/2013

Accepted: 18/4/2013

Keywords: Dendritic cells - Thymic selection - Tolerance

Abbreviations: cDC: conventional DC - mTEC: medullary TEC - nTreg: natural Treg - pDC: plasmacytoid DC . TEC: thymic epithelial cell . TSA: tissue-specific Ag

See Accompanying Viewpoint: http://dx.doi.org/10.1002/eji.201243154

The complete Cells in Motion Viewpoint series is available at:

http://onlinelibrary.wiley.com/doi/10. 1002/eji.v43.6/issuetoc 


\title{
Migration in the clinic
}

\section{Targeting cells in motion: Migrating toward improved therapies}

\author{
Jason W. Griffith ${ }^{1,2}$ and Andrew D. Luster ${ }^{1,3}$ \\ ${ }^{1}$ Center for Immunology and Inflammatory Diseases, Massachusetts General Hospital and \\ Harvard Medical School, Boston, MA, USA \\ ${ }^{2}$ Pulmonary and Critical Care Unit, Massachusetts General Hospital and Harvard Medical \\ School, Boston, MA, USA \\ ${ }^{3}$ Division of Rheumatology, Allergy \& Immunology, Massachusetts General Hospital and \\ Harvard Medical School, Boston, MA, USA
}

DOI: $10.1002 /$ eji.201243183

The development of clinical therapeutics that interfere with the migration of leukocytes has revolutionized the treatment of multiple sclerosis and holds great promise for the treatment of a wide range of inflammatory diseases. As the molecules essential for the multi-step adhesion cascade that mediates cellular migration have been elucidated, the number of potential targets available to modulate leukocyte trafficking has increased exponentially. In this Viewpoint, we briefly review our current understanding of these molecular targets and how these targets vary by tissue and leukocyte subset with emphasis on $\mathrm{T}$ cells. We then describe the two currently approved therapeutics that target cell migration, natalizumab and fingolimod, and discuss how an improved understanding of their function could pave the way for the development of safer and more efficacious therapies for inflammatory and autoimmune diseases.

\section{Introduction}

Nearly 50 years ago, Gowans and Knight published a seminal study demonstrating that labeled lymphocytes injected into rats migrated from the blood into secondary lymphoid organs (SLOs) and then returned to the circulation via the thoracic duct [1]. In an accompanying paper by Marchesi and Gowans, lymphocytes were observed to adhere to what are now called high endothelial venules and pass through the endothelial layer in a directed migration into the lymph node [2]. This pro- cess was hypothesized to be selective, as only small lymphocytes emigrated from the venules while larger lymphocytes were excluded. In the time since these first observations were made, knowledge of the molecular mechanisms that underpin lymphocyte trafficking has exploded. The selective migration observed by Marchesi and Gowans is now understood to be a tightly orchestrated multistep adhesion cascade, regulated by selectins, integrins, chemokines, and chemoattractant lipids, that specifically directs the trafficking of leukocytes into sites essential for their function. Such an improved understanding of the underlying mechanisms involved has resulted in the identification of an array of potential drug targets aimed at modulating cell migration in order to treat a broad range of autoimmune and inflammatory diseases. Today, two drugs targeting cell migration are approved for clinical use in multiple sclerosis, one of which is also approved for Crohn's disease; and many more are currently in clinical trial for these and other inflammatory diseases. In this Viewpoint, we will briefly discuss the wide range of molecular targets now recognized to inhibit leukocyte migration and our understanding that some of these targets may be unique to particular leukocyte subsets. We will then discuss two therapeutics that are currently in use for the inhibition of T-cell trafficking and how knowledge about their mechanism will inform the future development of drugs that target pathologic inflammation via the modulation of cell migration.

\section{Sticky targets}

The concept of a multistep adhesion cascade responsible for leukocyte extravasation has been an extremely successful framework for contextualizing the large array of molecules that participate in cell migration $[3,4]$. Currently, the leukocyte adhesion cascade is understood as a process of four successive steps: (i) leukocyte rolling along the endothelium, (ii) leukocyte activation, followed by (iii) adhesion onto endothelial cells and subsequent (iv) diapedesis into the target tissue [5]. The multistep adhesion cascade is driven by an overlapping but sequential interaction of a diverse group of adhesion and chemoattractant molecules $[6,7]$. The initial rolling step is mediated by the selectins, a three member family of C-type lectins, which bind with a high on/off rate to a wide range of sialylated carbohydrate ligands expressed on endothelial cells and the leukocytes themselves. This association then allows the circulating leukocyte to interact with regionally produced chemoattractant molecules. These chemoattractant molecules act to precisely control access of particular cell types to specific tissues and therefore are composed of a diverse group of lipids and chemokines that function in a combinatorial and likely nonredundant fashion in vivo [8].

Lipid chemoattractants include a relatively small number of eicosanoids, such as leukotriene $\mathrm{B}_{4}$, $\left(\mathrm{LTB}_{4}\right)$ and prostaglandin $\mathrm{D}_{2}\left(\mathrm{PGD}_{2}\right)$, and have recently been shown to initiate early inflammatory cell migration via activation of G-protein-coupled receptors (GPCRs) [9-11]. However, the most diverse group of chemoattractants is composed of the chemokines, which are a large group of over 50 secreted ligands. These interact with at least 20 members of the seven transmembrane spanning GPCR family to tightly regulate cell motility and adhesion under both resting and inflammatory conditions $[12,13]$. During leukocyte rolling, the interaction of chemokines with their coordinate GPCRs then activates the circulating cell via an "insideout" signal that changes the conformation 
of the integrins on the leukocyte surface from a low-to-high affinity state for its ligand [14]. Integrins are a family of heterodimers composed of a small number of $\alpha$ and $\beta$ subunits that interact with both ECM molecules, such as fibronectin, and cell surface molecules of the immunoglobulin family, such as intercellular adhesion molecule-1 (ICAM-1) and vascular cell adhesion molecule-1 (VCAM-1) that are expressed in a variety of cell types, including endothelial cells, fibroblastic reticular cells, dendritic cells (DCs), and lymphocytes $[13,15]$. The high affinity integrin interaction with its ligands allows for the arrest and adhesion of the leukocyte on the endothelial cell - a process that is necessary for the subsequent transmigration into the targeted tissue. Once leukocytes gain access to the appropriate tissue, they migrate to their particular targets along chemotactic or haptotactic gradients [16]. Finally, at their target site, the retention of leukocytes in the tissue is tightly controlled and for T cells and DCs, this process is regulated by the lysophospholipid shingosine 1-phosphate (S1P) and by the chemokine receptor CCR7 and its ligands CCL19 and CCL21 [17-20].

On $\mathrm{T}$ cells, the differential expression of particular combinations of selectins, chemokine receptors, and integrins on leukocytes is highly regulated and results in a directed trafficking of cellular subsets to particular organs and tissue beds. Naïve T cells, for example, largely express the chemokine receptor CCR7 and the selectin CD62L, which directs them to circulate through the SLOs where they are more likely to have a productive interaction with antigen and antigen-presenting cells [13]. Once activated by antigen, the activated effector $\mathrm{T}$ cells upregulate the expression of chemokine receptors that correspond and can react to the chemokine ligands produced in inflamed tissues. For $\mathrm{CD}^{+} \mathrm{T}$ cells, the combination of chemokine receptors that are upregulated correlates with the cell-differentiation program upon activation. Thus, CXCR3 and CCR5 are preferentially upregulated on Th1 cells while Th2 cells preferentially express CRTH2, CCR4, and CCR8 [21]. The Th17 subset preferentially expresses CCR6 [22], and T follicular helper cells express CXCR5 $[23,24]$. Memory T cells can be divided into $\mathrm{CCR}^{+}, \mathrm{CD}^{2} \mathrm{~L}^{\mathrm{hi}}$ central memory $\mathrm{T}$ cells that circulate in the SLOs and $\mathrm{CCR}^{-}{ }^{-} \mathrm{CD}^{2} \mathrm{~L}^{\text {lo }}$ effector memory T cells, which traffic to peripheral tissues [25]. Interestingly, among T effector memory cells there appears to be a difference in the expression of $\mathrm{P}$ and $\mathrm{E}$ selectins by CD4 and CD8 cells, resulting in further differences of localization and migration of these lymphocyte subsets within the memory population [26].

The site where antigen is encountered by the naïve cell also affects the expression of chemokine receptors and integrins, "imprinting" them to return to particular tissue beds. This process has been best characterized for the gut and skin but also may occur in the CNS and lung [27]. In the mesenteric lymph nodes and GALT, for example, DC-produced retinoic acid induces the expression of CCR9 and the integrin $\alpha_{4} \beta_{7}$ on effector memory $\mathrm{T}$ cells. As the ligands for CCR9 and $\alpha_{4} \beta_{7}$ (CCL25 and MAdCAM-1, respectively) are mainly expressed on endothelial cells in the venules of the small intestine, these effector memory $\mathrm{T}$ cells then specifically home to the gut $[28,29]$. In skin draining peripheral nodes, activated $\mathrm{CD}^{+} \mathrm{T}$ cells upregulate CLA, CCR4, and CCR10 and downregulate CCR9 and $\alpha_{4} \beta_{7}$, resulting in preferential homing back to the dermis and epidermis. Interestingly, another vitamin, vitamin D3, has been found to control this homing in part through downregulation of the gut homing $\alpha_{4} \beta_{7}$ integrin and upregulation of the epidermis-homing CCR10 [28, 30]. Thus, targeting particular chemokine receptors or integrins for pharmacologic blockade may allow for the selected modulation or inhibition of the migration of specific pathogenic subsets of $\mathrm{T}$ cells that traffic to an affected organ and cause disease. Despite some obstacles, this idea is quickly becoming reality as an array of drugs that inhibit or modulate cell migration are actively being studied in clinical trials (Table 1). Furthermore, two drugs, natalizumab and fingolimod, that target different aspects of T-cell migration (Fig. 1), have already been approved for use in the clinic.

\section{Natalizumab}

In 1992, merely 28 years after Gowans and Knight first observed the trafficking of lymphocytes [1], the group of Steinman and Karin reported that blockade of the integrin $\alpha_{4} \beta_{1}$ (VLA-4) with an antibody prevented EAE, a rodent model of multiple sclerosis (MS) [31]. Using an in vitro binding assay that allowed for the adhesion of lymphocytes and monocytes to vessels in brain sections to be visualized, this group tested a panel of antibodies directed against various integrins known to participate in the multistep adhesion cascade on brain sections from Lewis rats with EAE. They found that antibodies directed against the integrin subunits $\alpha_{4}$ or $\beta_{1}$ prevented lymphocyte and monocyte binding. They then demonstrated that the development of paralysis caused by injection of a $\mathrm{CD}^{+}{ }^{+} \mathrm{T}$-cell clone specific for myelin basic protein could be prevented by blockade of $\alpha_{4}$ integrin (Fig. 1) [31].

Based on these observations, a humanized monoclonal IgG4 antibody to $\alpha_{4}$ integrin called natalizumab (Tysabri, Biogen Idec, and Elan Pharmaceuticals) was developed and tested in clinical trials. Phase III clinical trials with relapsing-remitting MS patients demonstrated that, compared with a placebo, natalizumab reduced the risk of sustained progression of disability by $42 \%$ and the annualized relapse rate by $68 \%$ [32], and resulted in a $54 \%$ reduction in annualized relapse rates when given with IFN- $\beta$ [33]. After an interim one-year analysis of these trials, the FDA approved natalizumab in 2004 for relapsing forms of MS. Approval was also given for the shortterm treatment of Crohn's disease after it was demonstrated that some Crohn's disease patients treated with natalizumab had higher remission rates, as compared with those patients given a placebo, an effect presumably driven by natalizumab's ability to prevent leukocyte homing to the gut by blocking the $\alpha_{4} \beta_{7}$ integrin [34]. However, as cases of the rare but deadly disease progressive multifocal encephalopathy (PML) were identified in both MS and Crohn's patients taking natalizumab, the drug was pulled from the market for all the patients in 2005 only three months after approval. PML is a demyelinating disease of the white matter and is caused by opportunistic infection with the JC virus in immunocompromised hosts [35]. A review of all patients who had been treated with natalizumab during clinical trials for MS, Crohns' disease, and rheumatoid arthritis estimated the risk to be $1: 1000$ for the development of PML while on the drug [36]. Given this low risk and proven benefits, the drug was re-introduced as a monotherapy for relapsing MS and Crohn's disease in 2006 but the drug carries a black box warning and can only be prescribed in registered centers under the Tysabri Outreach: Unified Commitment to Health (TOUCH ${ }^{\circledR}$ ) program [37]. More recently, an analysis of 212 confirmed cases of PML that have occurred in the postmarketing setting have identified the risk for development of PML in MS patients 
Table 1. Status and indication of drugs currently in clinical trials that target molecules involved in cell migration.

\begin{tabular}{|c|c|c|c|c|}
\hline Target & Drugs $^{a)}$ & Company & Indication ${ }^{\mathrm{b})}$ & Status \\
\hline \multirow[t]{3}{*}{ CCR1 } & BX471 & Schering AG/Berlex & Pelvic pain & Phase 2 completed \\
\hline & CCX354 & ChemoCentryx & RA & Phase 2 completed \\
\hline & AZD-4818 & AstraZeneca & COPD & Phase 2 completed \\
\hline \multirow[t]{7}{*}{ CCR2 } & PF-04634817 & Pfizer & Diabetic nephropathy & Phase 2 recruiting \\
\hline & PF-04136309 & Pfizer & Osteoarthritis & Phase 2 completed \\
\hline & & & Pancreatic cancer & Phase 1 recruiting \\
\hline & MK-0812 & Merk & MS & Phase 2 completed \\
\hline & & & RA & Phase 2 failed \\
\hline & CCX-140 & ChemoCentryx & Diabetic nephropathy & Phase 2 recruiting \\
\hline & CNTO-888 & Centocor & IPF & Phase 2 completed \\
\hline \multirow[t]{2}{*}{ CCR3 } & Bertilimumab & Cambridge antibody technology & UC & Phase 2 planned \\
\hline & GW766944 & GlaxoSmithKline & Asthma & Phase 2 completed \\
\hline CCR4 & AMG-761 & Amgen & Asthma & Phase 1 recruiting \\
\hline \multirow[t]{3}{*}{ CCR5 } & Maraviroc & Pfizer & HIV, immune reconstitution & Active \\
\hline & GSK706769 & GlaxoSmithKline & RA & Phase 2 withdrawn \\
\hline & HGS1025 & GlaxoSmithKline & UC & Phase 1 withdrawn \\
\hline \multirow[t]{4}{*}{ CCR9 } & GSK 1605786A & GlaxoSmithKline & UC & Phase 3 recruiting \\
\hline & CCX282-B & ChemoCentryx & UC & Phase 2 completed \\
\hline & & & Crohn's & Phase 2 completed \\
\hline & & & Celiac & Phase 2 completed \\
\hline \multirow[t]{3}{*}{ CXCR2 } & GSK 1325756 & GlaxoSmithKline & COPD & Phase 1 completed \\
\hline & SB-6569333-AAA & GlaxoSmithKline & COPD & Phase 1 completed \\
\hline & & & $\mathrm{CF}$ & Phase 1 completed \\
\hline \multirow[t]{2}{*}{ CXCR3 } & MSX1100 & Bristol-Meyer Squibb & RA & Phase 2 completed \\
\hline & & & $\mathrm{UC}$ & Phase 2 completed \\
\hline \multirow[t]{3}{*}{ CXCR1/CXCR2 } & SCH 527123 & Schering-Plough & Psoriasis & Phase 2 completed \\
\hline & & & Asthma & Phase 2 completed \\
\hline & & & COPD & Phase 2 completed \\
\hline \multirow[t]{4}{*}{ CRTH2 } & ARRY-502 & Array BioPharma & Asthma & Phase 2 recruiting \\
\hline & QAV680 & Novartis & Allergic rhinitis & Phase 2 completed \\
\hline & QAW039 & Novartis & Asthma & Phase 2 recruiting \\
\hline & ADC3680B & Pulmagen Therapeutics & Asthma & Phase 2 completed \\
\hline CRTH2 and DP1 & AMG 853 & Amgen & Asthma & Phase 2 completed \\
\hline \multirow[t]{2}{*}{$\mathrm{LTB}_{4}$} & CP-195543 & Pfizer & $\mathrm{RA}$ & Phase 2 terminated \\
\hline & BIIL-284 & Boehringer Ingelheim & $\mathrm{CF}$ & Phase 2 terminated \\
\hline \multirow[t]{3}{*}{ S1P1 } & GSK 2018682 & GlaxoSmithKline & MS & Phase 1 completed \\
\hline & ACT-128800 & Actelion & MS & Phase 2 ongoing \\
\hline & & & Psoriasis & Phase 2 completed \\
\hline \multirow[t]{2}{*}{ Selectins $(E, P, L)$} & Bimosiamose & Revotar & COPD & Phase 2 completed \\
\hline & & & Psoriasis & Phase 2 completed \\
\hline \multirow[t]{3}{*}{ Integrin $\alpha_{4} \beta_{7}$} & Vedolizumab & Millennium & Crohn's & Phase 3 completed \\
\hline & AMG 181 & Amgen & UC & Phase 3 ongoing \\
\hline & & & UC & Phase 2 planned \\
\hline \multirow[t]{2}{*}{ Integrin $\alpha_{L} \beta_{2}$} & BMS-587101 & Bristol-Meyers Squibb & Psoriasis & Phase 2 terminated \\
\hline & MIRT 2584 & Boehringer Ingelheim & Psoriasis & Phase 2 suspended \\
\hline
\end{tabular}

a) This table does not list all compounds currently in development but instead summarizes drugs targeting the indicated molecules that are listed on www.clinicaltrials.gov.

b) RA: rheumatoid arthritis; COPD: chronic obstructive pulmonary disease; MS: multiple sclerosis; IPF: idiopathic pulmonary fibrosis; UC: ulcerative colitis; HIV: human immunodeficiency virus; CF: cystic fibrosis; SIP1: sphingosine-1 phosphate receptor subtype 1.

taking natalizumab and have stratified these risks based on seropositivity for JC virus, prior immunosuppressant use, and duration of treatment with natalizumab greater than 2 years [38]. Using this risk stratification, the authors estimated that a negative anti-JC virus antibody status had a risk of development of PML at 0.09 per 1000 natalizumab treated patients while patients with all three risk factors had an estimated incidence of 11.1 per 1000. In addition to the infectious complications, there have also been case reports of patients who develop a severe worsening of MS after drug initiation [39]. The cause for this decline is currently unclear, but it is hoped that further study of these side effects will allow for the 


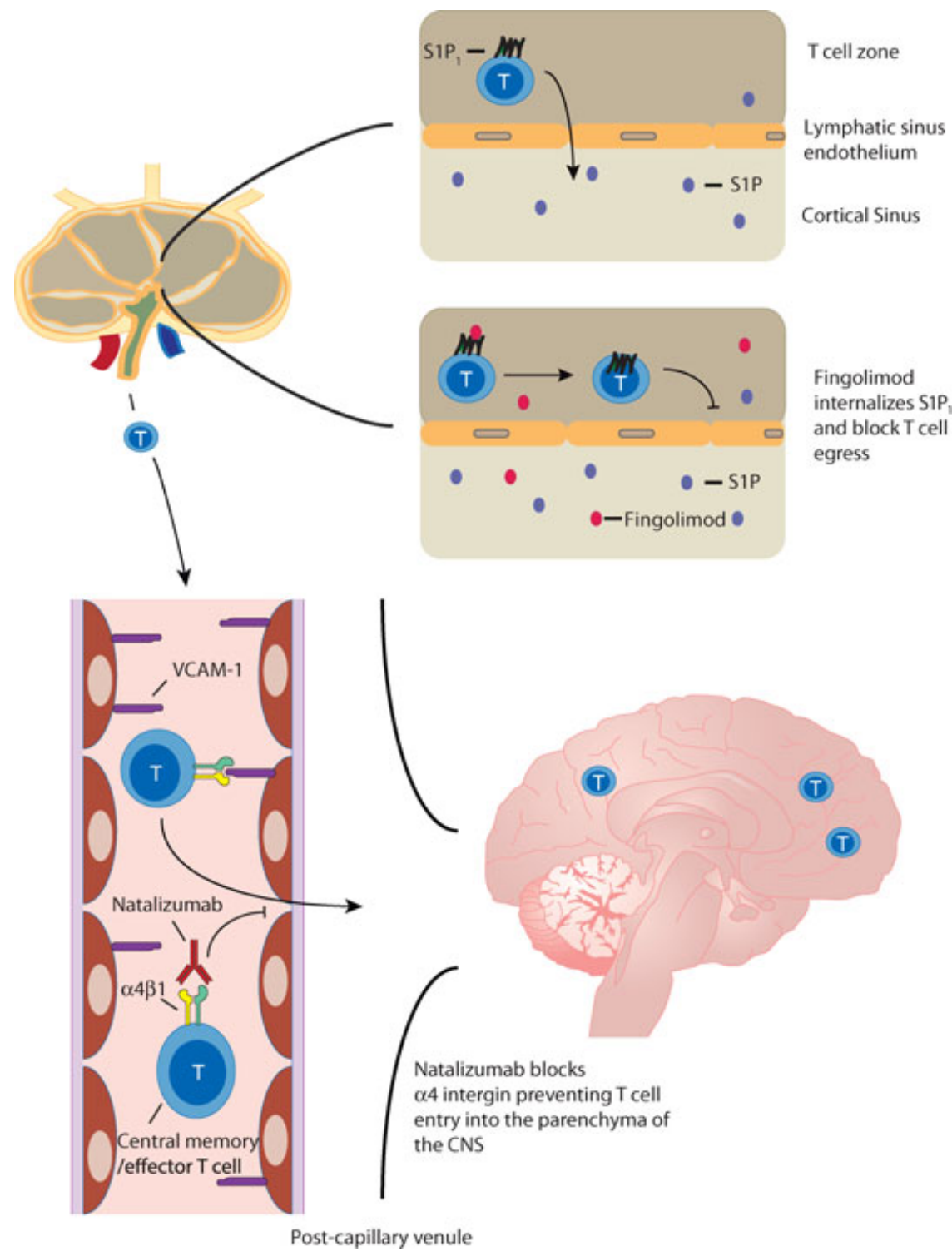

selection of only those patients who will safely benefit from natalizumab treatment.

\section{Fingolimod}

In the 1990s, a fungal metabolite with immunosuppressive properties was identified from culture filtrates of the ascomycete Isaria sinclairii [40], and subsequently chemically modified to a less toxic molecule termed FTY720. This molecule was originally thought to be a "classic" immunosuppressant that modulated $\mathrm{T}$ and B-cell activation as it was found to induce long-term graft acceptance in animal transplant models in synergy with calcineurin inhibitors [41]. However the idea that FTY720 was a "classic" immunosuppressant was challenged by observations that FTY720 did not inhibit the activation or proliferation of $\mathrm{T}$ and $\mathrm{B}$ cells [42] and the lack of therapeutic benefit compared with standard therapy in phase III trials of renal transplant rejection $[43,44]$ FTY720's mechanism of action became clear as studies demonstrated that FTY720 was an agonist of four out of the five known GPCRs for S1P, and it blocked lymphocyte egress from lymph nodes via downregulation and degradation of the $\mathrm{S}_{1 \mathrm{P}}$ receptor on lymphocytes (Fig. 1) $[17,45]$. Understanding the function of FTY720 revealed the critical importance of S1P gradients in mediating lymphocyte egress from the lymph node. This concept has been reinforced by studies that have demonstrated that disruption of the S1P gradient by inhibiting either S1P genera-
Figure 1. In MS patients, naïve $\mathrm{T}$ cells are thought to enter the lymph node (LN) where they encounter auto-antigens resulting in differentiation and activation into encephalitogenic effector $\mathrm{T}$ cells. During the later phases of activation, $\mathrm{T}$ cells upregulate $\mathrm{S}_{1} \mathrm{P}_{1}$, which then mediates $\mathrm{T}$-cell egress from the $\mathrm{LN}$ via migration toward the increased concentration of S1P present in the medullary sinus and efferent lymph (top right). Once these cells gain access to the circulation, they then adhere to endothelial cells in the CNS via the interaction of the integrin $\alpha 4 \beta 1$ on T cells with VCAM1 on the endothelial cell (bottom left). T cells then access the brain parenchyma where they become reactivated and secrete inflammatory cytokines and chemokines that recruit other effector cells resulting in the typical MS lesion in the white matter. In this sequence of events, fingolimod (red symbols) is thought to act via agonistic downregulation of the $\mathrm{S}_{1} \mathrm{P}_{1}$ receptor thereby blocking LN egress (top right) while natalizumab blocks the $\alpha 4 \beta 1$ integrin, effectively blocking the multistep adhesion cascade and $\mathrm{T}$-cell homing to the brain parenchyma (bottom left).

tion or its degradation inhibits lymphocyte egress from the lymph node [46,47].

As these discoveries came to light, the clinical effectiveness of FTY720 or fingolimod (Gilenya, Novartis) for the treatment of MS was studied in two large phase III clinical trials involving relapsing-remitting MS patients [48, 49]. Compared with a placebo, fingolimod decreased the annualized relapse rate by $54 \%$ [48], and when compared with IFN- $\beta$, fingolimod decreased the annualized relapse rate from 0.33 to 0.16 [49]. Thus, in September 2010 fingolimod was approved for use in patients with relapsing forms of MS. It should be noted that two deaths were reported in the trials $[48,49]$ but in patients taking a higher dose than that which is 
currently clinically approved. In one of these patients, disseminated primary varicella infection occurred during intravenous steroid treatment for relapse; in the other patient, herpes simplex encephalitis developed, also while the patient was on steroids. Other serious reported effects of fingolimod include bradycardia, a slight increase in lower respiratory tract infections, macular edema, and a reported increase in the development of skin and breast cancers. More recently, as seen with natalizumab, cases of paradoxical worsening of MS [50], or tumefactive MS [51], have been reported after initiation of fingolimod although the cause of these rare events is still unclear. Furthermore there have been more recent reports of serious herpes infections in patients taking fingolimod at the clinically approved dose $[52,53]$, reinforcing the need for further surveillance of safety [54]. Thus, patients treated with fingolimod will be followed by a 5-year postauthorization safety study to monitor for adverse events [55].

\section{Learning lessons and making them stick}

Although the approval of natalizumab and fingolimod represents the successful targeting of molecules that modulate cell migration, the explosion of knowledge about other cell migration targets, such as the chemokine receptors, has thus far been challenging to translate into new clinical therapeutics. The reasons for these disappointing results are numerous and have been thoroughly reviewed elsewhere recently $[8,56]$, but likely include "redundancy" of chemokine function, inadequate in vivo dosing, and the improper selection of targets as was suggested to have occurred in the clinical trials for CCR2 inhibition in rheumatoid arthritis [57]. We believe that an improved understanding of the mechanism and side effects of natalizumab and fingolimod will help address some of these obstacles. For instance, both of these drugs have highlighted the subtleties of modulating lymphocyte trafficking, such as only affecting particular subsets, subtleties that were not fully appreciated prior to their clinical approval.

Natalizumab, for instance, has been demonstrated to reduce the number of inflammatory cells in the cerebral spinal fluid of patients with MS, suggesting that it may indeed prevent the access of pathogenic $\mathrm{T}$ cells to the brain in humans [58]. This reduc- tion in inflammatory cells, however, is not global but appears to be relatively selective for particular leukocyte subsets. For example, the $\mathrm{CD}^{+} / \mathrm{CD}^{+} \mathrm{T}$-cell ratio is decreased in the cerebral spinal fluid [59], DC numbers are decreased in the perivascular spaces [60] and peripheral CD19 ${ }^{+}$B-cell and NK-cell numbers are increased [61] in natalizumab-treated MS patients. In addition, recent animal data using the EAE model demonstrated that blockade of $\alpha_{4}$-integrin is selective for Th1 cells and does not prevent the accumulation of pathogenic Th17 cells in the brain during disease $[62,63]$. As suggested by the authors of these studies, if confirmed in humans, this finding would imply that the majority of patients who respond to natalizumab therapy likely have a Th1mediated disease while patients who do not respond may have a predominately Th17-driven disease.

Fingolimod also appears to have differential effects on particular cellular subsets. For example, fingolimod selectively promotes the peripheral retention of naïve and central memory cells while having less effect on the homing of effector memory $\mathrm{T}$ cells in MS patients [64]. In particular, it has been shown that Th17 cells form a significant part of the central memory pool and numbers of these cells are reduced in the blood of MS patients taking fingolimod [65]. Although there have been conflicting reports about the action of fingolimod on regulatory $\mathrm{T}$ (Treg) cells $[66,67]$, it has been reported in mice that fingolimod differentially effects the trafficking of Treg cells as compared with CD25- CD4 ${ }^{+} \mathrm{T}$ cells [68]. In contrast, it appears that natalizumab has minimal effects on Treg cells [69]. Given these differential effects on T-cell subsets, it is tempting to speculate that the paradoxical worsening of MS that can occasionally be seen in patients taking fingolimod or natalizumab may be secondary to an inhibition of trafficking of a beneficial T-cell type such as Treg cells to the MS lesions or to an alteration of the balance of Th1/Th17 cells in MS lesions; however, confirmation of this theory awaits further clinical study.

To sum up, the data obtained from studying the effects of natalizumab and fingolimod suggest that cell migration inhibitors may have very specific and differential effects on lymphocyte subsets that may be difficult to predict without further study. As more drugs that inhibit migration progress through clinical trials for diseases as diverse as COPD, asthma, rheumatoid arthritis, MS and Crohn's, the reports of devastating infections in patients on natalizumab and fingolimod should also give us pause for thought. Somewhat surprisingly, current reports suggest that natalizumab and fingolimod each increase the risk of a specific but different type of infection - natalizumab increases the risk for PML [35] while fingolimod may be associated with a slightly increased risk for herpes infections, although this risk needs to be confirmed with further postmarketing surveillance $[52,53]$. These observations should arouse caution when contemplating the inhibition of similar cellular subsets as those targeted by natalizumab and fingolimod. For instance, if it is confirmed that natalizumab selectively inhibits the accumulation of Th1 cells in the CNS of patients, then other cell migration inhibitors that target Th1 cells, such as inhibitors of CXCR3 and CCR5, should be carefully assessed for the risk of similar infectious complications, including the development of PML. Likewise, as fingolimod appears to selectively inhibit naïve and central memory cells, including those cells differentiated into a Th17 subset, vigilance for similar infections to those observed for fingolimod - namely herpes infections - should be high when undertaking clinical trials of migration inhibitors that target these subsets.

Finally, the effects of these drugs beyond their modulation of cell migration add complexity to understanding the clinical response that they induce. For instance, natalizumab induces the release of immature $\mathrm{CD}_{3} 4^{+}$leukocytes from the bone marrow [70], impairs the ability of DCs to stimulate antigen-specific T-cell responses [71], and could potentially block VLA-4's ability to synergize with TCR signaling to augment T-cell stimulation and proliferation [72, 73]. In contrast, fingolimod has effects on vascular permeability, mast cell activation, astrocyte susceptibility to apoptosis, and cardiomyocyte function [74]. Teasing apart these effects from those affecting T-cell migration will be challenging but will nonetheless likely improve our understanding of the exact mechanisms of action of cell migration inhibitors proposed for therapeutic use.

\section{Future prospects}

The successful clinical implementation of natalizumab and fingolimod provides proof that modulating cell migration is an effective means to modulate inflammation. 
The explosion of knowledge about the molecules that mediate the cell migration of leukocytes has resulted in a significant number of new targets that hold promise for new therapies [4, 56, 75]. However, as the drugs natalizumab and fingolimod demonstrate, we still need to refine our understanding of the molecules that are important for the trafficking of specific lymphocyte subsets in humans and how these subpopulations mediate disease and resistance to infection. As more drugs enter the pipeline, this knowledge should allow for a better prediction of clinical benefit and the possible infectious complications of treatment with cell migration inhibitors and allow for strategies to maximize clinical effectiveness while minimizing the risks of this promising class of drugs.

Acknowledgments: J.W.G. was supported by an NHLBI/NIH T32 training grant and A.D.L. was supported by grants from the NIAID and the NCI at the NIH.

Conflict of interest: The authors declare no financial or commercial conflict of interest.

\section{References}

1 Gowans, J. L. and Knight, E. J., Proc. R. Soc. Lond. B. Biol. Sci. 1964. 159: 257-282.

2 Marchesi, V. T. and Gowans, J. L., Proc. R. Soc. Lond. B. Biol. Sci. 1964. 159: 283-290.

3 Butcher, E. C., Cell 1991. 67: 1033-1036.

4 Mackay, C. R., Nat. Immunol. 2008. 9: 988-998.

5 Ley, K. et al., Nat. Rev. Immunol. 2007. 7: 678689.

6 Luster, A. D. et al., Nat. Immunol. 2005. 6: 1182-1190.

7 Springer, T. A., Cell 1994. 76: 301-314.

8 Schall, T. J. and Proudfoot, A. E., Nat. Rev. Immunol. 2011. 11: 355-363.

9 Sadik, C. D. and Luster, A. D., J. Leukoc. Biol. 2012. 91: 207-215.

10 Tager, A. M. et al., Nat. Immunol. 2003. 4: 982-990.

11 Luster, A. D. and Tager, A. M., Nat. Rev. Immunol. 2004. 4: 711-724.

12 Schaerli, P. and Moser, B., Immunol. Res. 2005. 31: 57-74.

13 Bromley, S. K. et al., Nat. Immunol. 2008. 9: 970-980.

14 Kinashi, T., Nat. Rev. Immunol. 2005. 5: 546-559.

15 Springer, T. A., Annu. Rev. Physiol. 1995. 57: 827-872.

16 Friedl, P. and Weigelin, B., Nat. Immunol. 2008. 9: 960-969.

17 Matloubian, M. et al., Nature 2004. 427: 355-360.
18 Bromley, S. K. et al., Nat. Immunol. 2005. 6: 895-901.

19 Debes, G. F. et al., Nat. Immunol. 2005. 6: 889-894.

20 Forster, R. et al., Cell 1999. 99: 23-33.

21 Syrbe, U. et al., Springer Semin. Immunopathol. 1999. 21: 263-285.

22 Acosta-Rodriguez, E. V. et al., Nat. Immunol. 2007. 8: 639-646.

23 Breitfeld, D. et al., J. Exp. Med. 2000. 192: 1545-1552.

24 Schaerli, P. et al., J. Exp. Med. 2000. 192: 1553-1562.

25 Sallusto, F. et al., Nature 1999. 401: 708-712.

26 Gebhardt, T. et al., Nature 2011. 477: 216-219.

27 Agace, W. W., Nat. Rev. Immunol. 2006. 6: 682-692.

28 Sigmundsdottir, H. and Butcher, E. C., Nat. Immunol. 2008. 9: 981-987.

29 Salmi, M. and Jalkanen, S., Immunol. Rev. 2005. 206: 100-113.

30 Kupper, T. S. and Fuhlbrigge, R. C., Nat. Rev. Immunol. 2004. 4: 211-222.

31 Yednock, T. A. et al., Nature 1992. 356: 63-66.

32 Polman, C. H. et al., N. Engl. J. Med. 2006. 354 899-910.

33 Rudick, R. A. et al., N. Engl. J. Med. 2006. 354: 911-923.

34 Ghosh, S. et al., N. Engl. J. Med. 2003. 348: 24-32.

35 Major, E. O., Annu. Rev. Med. 2010. 61: 35-47.

36 Yousry, T. A. et al., N. Engl. J. Med. 2006. 354: 924-933.

37 Ransohoff, R. M., N. Engl. J. Med. 2007. 356: 2622-2629.

38 Bloomgren, G. et al., N. Engl. J. Med. 2012. 366: 1870-1880.

39 Rinaldi, F. et al., Mult. Scler. 2009. 15: 1359-1362.

40 Fujita, T. et al., J. Antibiot. 1994. 47: 208-215.

41 Suzuki, S. et al., Transplantation 1996. 61: 200-205.

42 Brinkmann, V. et al., Transplant. P. 2001. 33: 530-531.

43 Hoitsma, A. J. et al., Nephrol. Dial. Transpl. 2011. 26: 3802-3805.

44 Tedesco-Silva, H. et al., Transplantation 2006. 82: 1689-1697.

45 Mandala, S. et al., Science 2002. 296: 346-349.

46 Pham, T. H. et al., J. Exp. Med. 2010. 207: 17-27.

47 Schwab, S. R. et al., Science 2005. 309: 1735-1739.

48 Kappos, L. et al., N. Engl. J. Med. 2010. 362: 387-401.

49 Cohen, J. A. et al., N. Engl. J. Med. 2010. 362: 402-415.

50 Centonze, D. et al., Neurology 2012. 79: 2004-2005.

51 Visser, F. et al., Neurology 2012. 79: 2000-2003.

52 Gross, C. M. et al., Neurology 2012. 79: 2006-2007.

53 Ratchford, J. N. et al., Neurology 2012. 79: 2002-2004.
54 Bourdette, D. and Gilden, D., Neurology 2012. 79: 1942-1943.

55 Hohlfeld, R. et al., Neurology 2011. 76: S28-37.

56 Allegretti, M. et al., Immunol. Lett. 2012. 145: 68-78.

57 Proudfoot, A. E., Arthritis Rheum. 2008. 58: 1889-1891.

58 Stuve, O. et al., Ann. Neurol. 2006. 59: 743-747.

59 Stuve, O. et al., Arch. Neurol. 2006. 63: 1383-1387.

60 del Pilar Martin, M. et al., Arch. Neurol. 2008. 65: 1596-1603.

61 Harrer, A. et al., PLoS One 2012. 7: e31784.

62 Rothhammer, V. et al., J. Exp. Med. 2011. 208: 2465-2476.

63 Glatigny, S. et al., J. Immunol. 2011. 187: 6176-6179.

64 Mehling, M. et al., Neurology 2008. 71: 1261-1267.

65 Mehling, M. et al., Neurology 2010. 75: 403-410.

66 Wolf, A. M. et al., J. Immunol. 2009. 183: 3751-3760.

67 Daniel, C. et al., J. Immunol. 2007. 178: 2458-2468.

68 Sawicka, E. et al., J. Immunol. 2005. 175: 7973-7980.

69 Stenner, M. P. et al., PLoS One 2008. 3: e3319.

70 Zohren, F. et al., Blood 2008. 111: 3893-3895.

71 de Andres, C. et al., PLoS One 2012. 7: e34103.

72 Dang, N. H. et al., J. Exp. Med. 1990. 172: 649-652.

73 Sato, T. et al., J. Immunol. 1995. 155: 2938-2947.

74 Rosen, H. and Goetzl, E. J., Nat. Rev. Immunol. 2005. 5: 560-570.

75 Salmi, M. and Jalkanen, S., Eur. J. Immunol. 2012. 42: 284-292.

Correspondence: Prof. Andrew D. Luster, Massachusetts General Hospital, Building 149, 13th Street, Charlestown, MA 02129, USA Fax: +1-617-726-5615

e-mail: aluster@mgh.harvard.edu

Received: 21/11/2012

Revised: 31/3/2013

Accepted: 8/4/2013

Keywords: Cell migration - Cell trafficking • Chemokines

Abbreviations: GPCR: G protein-coupled receptor PML: progressive multifocal encephalopathy - SLO: secondary lymphoid organ - S1P: shingosine 1-phosphate

See accompanying Viewpoint: http://dx.doi.org/10.1002/eji.201243155

The complete Cells in Motion Viewpoint series is available at: http://onlinelibrary.wiley.com/doi/10. 1002/eji.v43.6/issuetoc 


\title{
Defects of leukocyte migration in primary immunodeficiencies
}

\author{
Raffaele Badolato \\ Department of Pediatrics, Institute of Molecular Medicine "Angelo Nocivelli", University of \\ Brescia, Brescia, Italy
}

DOI: 10.1002/eji.201243155

This Viewpoint gives an overview of the inherited disorders that are characterized by defects of leukocyte trafficking. Three paradigmatic diseases have been selected: warts, hypogammaglobulinemia, infections, and myelokathexis; WiskottAldrich; and leukocyte adhesion deficiency syndromes. These conditions encompass defects in the steps that are required for leukocyte motility: from the response to chemokines, which is altered in warts, hypogammaglobulinemia, infections, and myelokathexis syndrome, to the impairment of leukocyte adhesion and migration found in leukocyte adhesion deficiency syndrome, and finally to abnormal actin filament formation, which is affected in leukocytes of Wiskott-Aldrich syndrome patients.

\section{Introduction}

Leukocyte motility in response to chemokines is required for the inflammatory response to infections, and for organ development, tissues homeostasis, and vascularization. The role in infections is clearly seen in those inherited disorders that affect the key players involved in leukocyte motility with patients with such disorders displaying susceptibility to infections. Although many primary immunodeficiencies are associated with defects in leukocyte trafficking, this Viewpoint focuses on the warts, hypogammaglobulinemia, infections, and myelokathexis (WHIM); Wiskott-Aldrich; and leukocyte adhesion deficiency (LAD) syndromes as the leukocyte migration defects in these disorders have been extensively characterized.

CXCR4 mutations in WHIM syndrome

WHIM syndrome represents the first identified example of a human disease that is caused by the abnormal functioning of a chemokine receptor [1-3]. This condition is a genetic disorder usually inherited as autosomal dominant disease with a prevalence $<1: 1000000$ due to heterozygous mutations of the gene encoding for CXCR4, the receptor for the CXCL12 chemokine (Table 1). All the mutations that have been identified so far affect the cytosolic carboxy-terminal (CT) tail of CXCR4, resulting in loss or inactivation of the function of 10-19 amino acids in the CT tail, thus leading to an enhanced and prolonged response to CXCL12 [4-7]. Given the broad expression of CXCR4, especially in stem and progenitor cells such as hematopoietic, neural and liver stem cells, primordial germ cells, skeletal muscle, and retinal progenitor cells, with the abnormal functioning of the receptor one might expect multiple defects in the nervous and cardiovascular systems; however, despite this broad CXCR4 expression pattern, the main clinical features of WHIM syndrome are restricted to the hematopoietic organs and, in particular, the generation of myeloid cells from the bone marrow, their recirculation, and the homeostasis of lymphocyte subsets.

Although most WHIM patients display leukopenia, WHIM syndrome is usually only suspected after the discovery of neutropenia and monocytopenia, usually the first manifestation of the disease $[4,5,8]$. Analysis of bone marrow in these patients reveals myeloid hypercellularity, despite severe neutropenia in peripheral blood, with the mature neutrophils displaying morphologic abnormalities such as cytoplasmatic vacuolization, hypersegmented nuclei, and chromatin hypercondensation. Neutrophils are retained in the bone marrow of WHIM patients because of the heightened response of these cells to the CXCR4 ligand CXCL12. Indeed, the constitutive expression of CXCL12 by hematopoietic stromal cells, which is critical for the retention of stem and progenitor cells in the bone marrow during hematopoiesis, leads to the entrapment of mature neutrophils in the same location $[2,9,10]$. Nonetheless, the neutrophils of WHIM patients can be mobilized from the bone marrow to peripheral blood during infections and display normal biologic functions, despite their morphologic abnormalities.

Patients with WHIM syndrome have difficulties in dealing with bacterial pathogens and typically develop infections of the respiratory tract with sinusitis and pneumonia being first manifestations of the disease; however, meningitis, osteomyelitis, deep soft tissue abscesses, urinary tract infections, and skin infections have also been observed. Although the majority of WHIM patients survive through adulthood, $70 \%$ of WHIM patients show extreme susceptibility to human papilloma virus (HPV) infection, which manifests as warts, especially on the hands, feet, and trunk and as anogenital condylomata acuminate which may secondarily lead to dysplastic HPV-induced lesions and invasive cancer. The warts, however, are not present at the onset of WHIM syndrome but appear during the first or second decade of life $[4,5]$.

Besides recurrent infections and an increased risk of tumors, WHIM patients display a higher rate of congenital heart defects. In particular, three cases of tetralogy of Fallot have been recently reported in patients with this condition, suggesting that heterozygous mutations in CXCR4 can be associated with defects in organogenesis [11]. This is in keeping with studies in mice that have demonstrated a key role for CXCL12 and CXCR4 in heart, nervous system, and blood vessel development [12], and, possibly in concert with CXCR7, the recently described second receptor for CXCL12, in heart valve development [13].

Immunological features associated with truncating mutations of CXCR4

Initial studies showed that neutrophils and lymphocytes from patients with WHIM syndrome display increased chemotaxis, adhesion, and signaling in response to CXCL12, suggesting that neutropenia might result from the impairment of neutrophil egression from the bone marrow 
Table 1. Inherited disorders associated with abnormal leukocyte motility.

\begin{tabular}{llllll}
\hline & Leukopenia & Leukocytosis & T-cell defect & B-cell defect & Locus \\
\hline WHIM syndrome & + & - & + & + & CXCR4 \\
Classical Wiskott-Aldrich syndrome (WAS) & + & - & + & + & WAS \\
X-linked thrombocytopenia (XLT) & - & - & - & - & WAS \\
X-linked neutropenia (XLN) & + & - & - & + & WAS \\
WIP deficiency & + & - & + & - & WIP \\
Coronin 1A deficiency & + & - & + & - & CORO1A \\
Leukocyte adhesion deficiency I & - & + & - & ITGB2 \\
Leukocyte adhesion deficiency II & - & + & + & FEC35C1 \\
Leukocyte adhesion deficiency III & - & + & + & RAC2 \\
Rac2 deficiency & - & + & + & & \\
\hline
\end{tabular}

$[2,3,14]$. Subsequent reports have given direct support to this hypothesis in mouse and zebrafish models of WHIM syndrome and more recently in a new knock-in mouse strain with a heterozygous mutation in CXCR4. These animal models have shown that CXCR4 mutations associated with WHIM syndrome lead to leukopenia and impaired neutrophil motility and recruitment to wounds $[9,10,15]$. The abnormal recruitment of neutrophils accounts for the recurrence of pyogenic infections in patients with WHIM syndrome, but does not explain the high risk of disseminated HPV infection that is frequently observed in these subjects.

In many cases, patients with WHIM syndrome present lymphopenia and hypogammaglobulinemia. In particular, the absolute counts of both $\mathrm{T}$ and $\mathrm{B}$ cells are reduced, with a profound reduction in circulating memory B cells that affects both unswitched $\left(\operatorname{IgD}^{+}\right)$and switched $\left(\operatorname{IgD}^{-}\right)$ CD19 B lymphocytes [2, 16, 17]. T-cell lymphopenia is probably due to abnormal motility of these cells between lymphoid organs, but is probably also related to reduced thymic output since circulating $\mathrm{T}$ cells have a decreased number of TCR excision circles [2]. Analysis of $\mathrm{B}$ cells and of the immunoglobulin response to antigens, including bacteriophage $\Phi \mathrm{X} 174$, has revealed oligoclonality of circulating B cells and abnormal isotype switching, probably due to impairment of B-cell trafficking within LNs [17]. This is in accordance with the analysis of inguinal LNs in two patients with WHIM syndrome who had either no or poorly formed lymphoid follicles and reduced numbers of plasma cells [18]. Despite the oligoclonality of the B-cell repertoire in the peripheral blood of WHIM syndrome patients, hypogammaglobulinemia is not a constant feature of WHIM syndrome since immunoglobulin levels are normal or slightly reduced in more than half of the patients $[5,8]$, suggesting that tissueresident $B$ cells contribute to antibody secretion.

The abnormal motility of both $\mathrm{T}$ and $B$ cells might have a role in the increased susceptibility of WHIM patients to HPV infections (Table 1). However, other cells types, such as plasmacytoid dendritic cells (pDCs), whose generation and motility are in part dependent on CXCR4, might also be involved. In particular, analysis of this subset has shown a striking defect in the number of pDCs in the peripheral blood of WHIM patients and in skin biopsies of one WHIM patient [19]. Moreover, the production of type I interferon by mononuclear cells in response to herpesvirus infection was undetectable in WHIM patients [19]. Given that pDCs are important for defense against HPV, it is probable that their depletion contributes to the susceptibility of WHIM patients to warts.

Treatments of patients with WHIM include intravenous immunoglobulin infusions and subcutaneous therapy with GCSF, both of which are usually effective at preventing recurrent bacterial infections, but do not lend protection against HPV infection and the associated tumors of the genitourinary tract $[5,8]$. These limitations might be overcome in the future by the introduction of the CXCR4 antagonist plerixafor (AMD3100), a drug that has been approved for hematopoietic stem cell mobilization. Recently, two phase 1 clinical trials in adult WHIM patients have shown that plerixafor administration results in increased leukocyte counts in the blood of patients with WHIM syndrome, suggesting its potential use as treatment for these subjects $[16,20]$. Nonetheless, these encouraging results need to be confirmed in a larger cohort of patients.

\section{Wiskott-Aldrich syndrome protein (WASP) associated diseases}

Wiskott-Aldrich syndrome protein (WASP) constitutes the first discovered member of a family of proteins that regulate dynamic changes in the actin cytoskeleton, but is the only member of this family with expression restricted to the hematopoietic compartment. WASP is a cytoplasmic protein that is devoid of catalytic activity, but serves as a scaffold to convey a variety of signals from membranes or intracellular proteins to the cytoskeleton [21]. WASP is usually present in the cytoplasm in an auto-inhibited form in which the verprolin homology domain (known as the VCA domain) located at the CT binds with a hydrophobic region within the GTPase-binding domain (GBD) (Fig. 1A). Following activating signals, such as triggering of the $\mathrm{T}$-cell receptor, the Rho family GTPase CDC42 is able to interact with the WASP GBD, inducing the transition of WASP to an active form by releasing the CT from auto-inhibition. This transition can be activated by phosphorylation of tyrosine Y291 that is located within the GBD. Thereafter, the VCA region is free to bind a complex of actin-related proteins and recruit monomeric actin to generate branched actin filaments. The WASP homology 1 (WH1) domain, which is located at the amino-terminus, is not involved in signaling but functions to stabilize the protein by binding with WASP interacting protein (WIP) to prevent its degradation by proteases (Fig. 1B) [22].

Mutations of WASP or of WIP can lead to four related diseases (classical WiskottAldrich syndrome (WAS), X-linked 


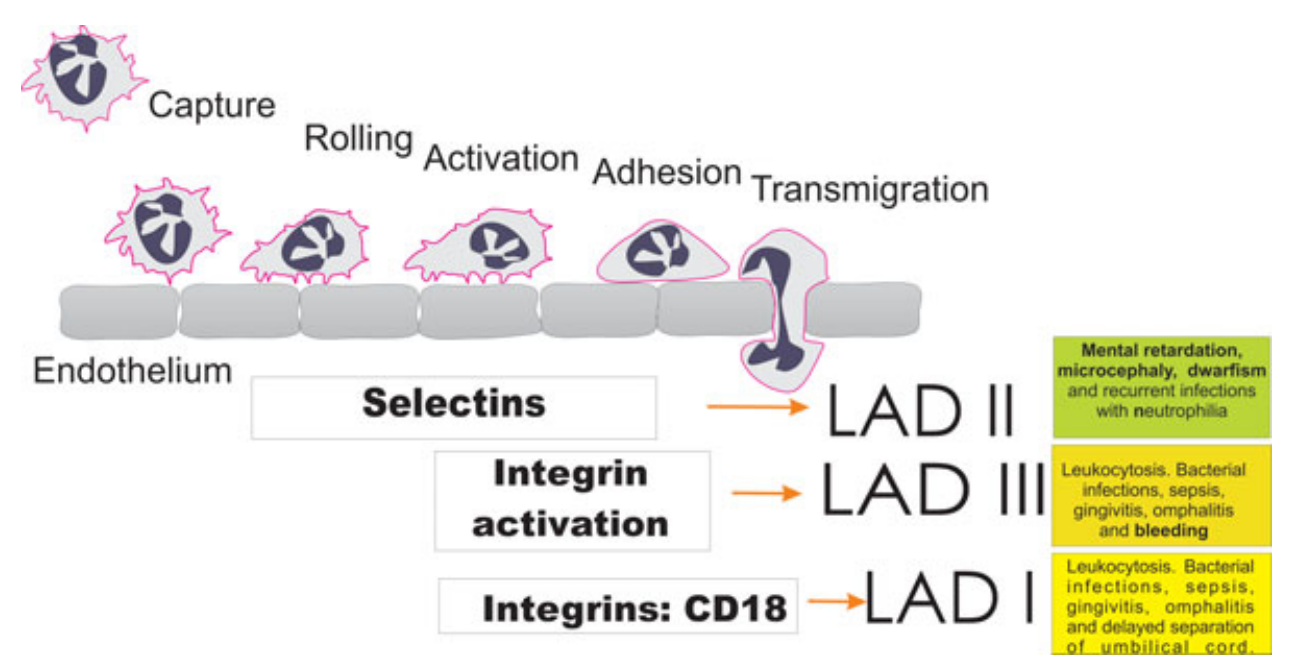

Figure 1. WAS protein structure and disease mutations. (A) WAS protein is a cytosolic protein that can be present in an auto-inhibited form. In this form, the VCA binds with a hydrophobic region within the GBD and is stabilized by WIP interaction with the WH1 domain, which is located at the amino-terminus. (B) WASP becomes active by releasing the CT from auto-inhibition. This process can be activated by phosphorylation of the tyrosine residue (Y291) located at the hydrophobic pocket of the GBD. Thereafter, the VCA region is free to bind the actin-related proteins complex and recruit monomeric actin to generate branched actin filaments (C) Mutations in exons 1-3 are usually missense mutations or splicing alterations that are located in the WH1 domain and lead to the low levels expression of WASP protein and are associated with XLT. The mutations associated with classical WAS include nonsense mutations, deletions, splicing alterations, and missense mutations outside exons 1-3 and usually result in either absence of the protein or loss of WASP functional activity. In contrast, XLN patients present mutations that affect the hydrophobic pocket of the GBD.

thrombocytopenia (XLT), X-linked neutropenia (XLN) and WIP deficiency) that are characterized by distinct clinical features and differences in WASP expression and function (Table 1 and Fig. 1C). WASP gene mutations were originally identified in patients with classical WAS, an X-linked disorder characterized by congenital microthrombocytopenia, moderate-to-severe eczema, and recurrent or severe bacterial infections, especially with encapsulated pathogens. These patients may also develop autoimmune manifestations and hematopoietic cell malignancy. Mutations that are associated with classical WAS include nonsense mutations, deletions, splicing alterations, and missense mutations outside exons 1-3 that encode for the WH1 domain and usually result in either absence of the protein or loss of WASP functional activity [23]. XLT is caused by mutations in the same gene, but the mutations in this case are usually missense mutations or splicing alterations that are located in the WH1 domain and lead to the expression of WASP protein but at lower levels [24, 25]. Missense mutations in the WASP WH1 domain affect its interaction with WIP and lead to the accelerated degradation and reduced WASP protein levels in cells from XLT patients, although the mutant protein retains the capacity to support actin polymerization [24]. XLT patients usually present only bleeding and microthrombocytopenia, but not recurrent infections, suggesting that generation of platelets is strictly dependent on WASP expression. Recently, Lanzi et al. [26] have reported on a patient with WIP deficiency and hence reduced WASP protein levels because of increased WASP degradation, whose clinical manifestations are partially reminiscent of WAS. XLN has been observed in a limited number of patients who present mutations that affect the hydrophobic pocket of the GBD required for VCA binding in the auto-inhibited form of WASP where the tyrosine residue 291 is located (Fig. 1) [27, 28]. These mutations confer constitutive WASP activation and promote diffuse actin polymerization instead of localized actin filaments at the plasma membrane, resulting in mitosis defects and apoptosis that ultimately lead to myelodysplasia and neutropenia [29].

\section{Adaptive and innate immunity defects in WAS patients}

Patients with WAS may display a number of immunological defects, including lymphopenia with preferential reduction of naïve T-cell numbers, poor antibody response to antigens, defective immunological synapse formation, and impaired migration of monocytes, macrophages, lymphocytes, and dendritic cells (DCs). In particular, cells of the myeloid lineage such as monocytes, macrophages, DCs, and osteoclasts from WAS patients display major defects in the formation of actin-rich microfilament bundles such as podosomes, impaired adhesion, and directional migration in response to CCL2, CCL3, fMLP, and CSF-1 or to the CCR7 ligands CCL19 and CCL21 [30-34]. Indeed, DC homing to T-cell zones of lymphoid organs and type I interferon production are impaired in Wasp-deficient mice [35-37]. Delayed migration of DCs and Langerhans cells from skin to LNs might lead to ectopic maturation of DCs in peripheral tissues resulting in increased inflammation of tissues, such the eczema observed in some WAS patients [21]. Although neutropenia is not common in WASP patients, neutrophil migration is also abnormal. In fact, WASP deficiency leads to global defects in directed leukocyte migration in vitro and in vivo $[38,39]$ as WASP regulates the reorganization of $\mathrm{CD} 11 \mathrm{~b}$ integrin at the uropod, which is critical for maintaining neutrophil polarity during migration [40].

Because WASP deficiency leads to abnormal actin filament formation in response to TCR stimulation, T cells from WASP-deficient patients show impaired immunological synapse formation and 
Auto-inhibited WASP

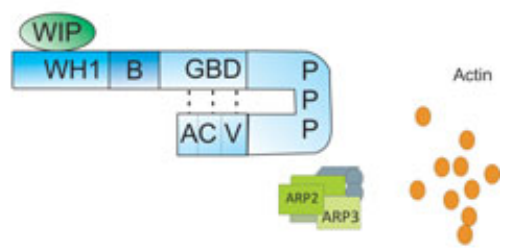

B

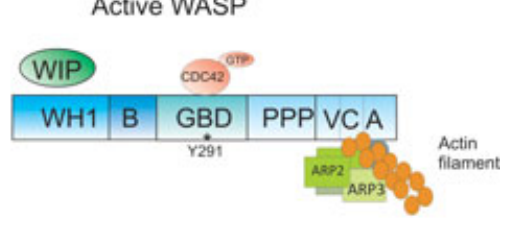

C

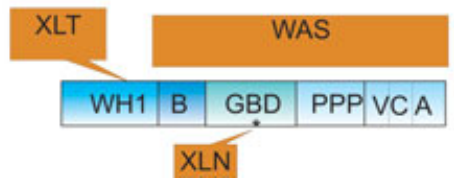

Figure 2. Leukocyte migration and LAD defects. Leukocyte migration to tissues constitutes a multistep process (top) consisting of capture, rolling activation, adhesion, and transmigration. Leukocyte rolling requires the expression of Sialyl Lewis X and other fucosylated selectin ligands that are not synthesized in LAD II patients. In the following step, integrins need to be activated, resulting in adhesion and transendothelial migration. Expression of beta2 integrins is impaired in LAD I, while integrin activation is defective in LAD III, resulting in abnormal adhesion and chemotaxis. Symptoms associated to LAD are summarized on the right. Typical symptoms associated to LAD II or LAD III are typed in bold.

polarized cytokine secretion. Moreover, WASP-deficient $\mathrm{T}$ cells migrate poorly in response to the $\mathrm{T}$-cell chemoattractants CXCL12 and CXCL19 that might account for their abnormal homing to lymphoid organs after adoptive transfer in mice $[38,41]$. Interestingly, $\mathrm{T}$ cells from one WIP-deficient patient also showed reduced chemotaxis, probably related to the decreased levels of WASP protein [26]. Impaired B-cell migration in response to sphingosine 1 phosphate might account for abnormal homeostasis of B cells and secondarily for the poor response to encapsulated bacteria in WAS patients [42, 43]. Mice lacking WASP and the neuronal Wiskott-Aldrich syndrome protein in B cells showed an even more pronounced defect in B-cell migration [44]. Finally, the observation that carriers from $\mathrm{X}$-linked WAS patients display nonrandom
$\mathrm{X}$-chromosome inactivation of $\mathrm{CD} 34^{+}$cells has been linked to a possible migration impairment of Wasp-deficient stem cells from fetal liver to bone marrow [45], although this hypothesis is challenged by other findings [42].

Impaired leukocyte adhesion and migration in CD18-deficient patients

LAD I constitutes the first defect of leukocyte trafficking and was identified about 30 years ago [46]. It is inherited as an autosomal recessive disorder due to mutations in the gene ITGB2 encoding the common beta-chain of the beta2 integrin family (CD18). CD18 can form three heterodimers by binding with the alpha subunits CD11a, CD11b, or CD11c, but the absence or abnormal synthesis of CD18 prevents cell surface expression of CD11 subunits as well. Most of the mutations of ITGB2 lead to the absence or reduced expression of CD18 on the cell surface as measured by flow cytometry [47]. In the most severe form of the disease, less than $2 \%$ of cells express CD18, while in the moderate form, the expression ranges between 2 and 30\%, which usually correlates with a lower number of infections [48]. Expression of the CD11/CD18 complex on the surface of leukocytes is required for many leukocyte activities including phagocytosis and cell-mediated cytotoxicity, but is especially needed for adhesion and transendothelial migration (Fig. 2). In particular, each one of the beta2 integrins binds one or more members of ICAM family in the firm adhesion of leukocytes to vascular endothelium [49]. In LAD I patients, leukocyte firm adhesion to the endothelium is defective and their migration to infected tissues is severely impaired $[47,50]$. The adhesion defect accounts for the increased risk of bacterial infections localized to skin and mucosal surfaces without pus formation and for the high frequency of omphalitis and delayed separation of umbilical cord that usually are the first manifestation of the disease. Periodontitis and gingivitis are commonly observed in the second decade of life.

The disease is usually suspected in patients who present with moderate neutrophilia in the absence of overt infection and marked granulocytosis (PMN counts above $100000 / \mathrm{mL}$ ) during acute infection $[47,48]$. Hematopoietic stem cell transplantation constitutes the only cure for patients with severe LAD I [51], while patients with residual CD18 expression can survive without HCST.

\section{Disorders of protein glycosylation or integrin activation lead to leukocyte adhesion deficiency type II and III}

LAD II is a rare form of leukocyte deficiency that is caused by a defect of fucose metabolism and is also known as congenital disorder of glycosylation type IIc. It is an autosomal recessive condition caused by mutations of the SLC35C1 gene that encodes for a GDP-fucose transporter protein localized to the Golgi apparatus $[52,53]$. Given that GDP-fucose transporter protein is required for the transport of GDP-fucose from the cytosol into the lumen of the Golgi, mutations of the gene leading to improper location or defective function prevent the incorporation of fucosyl residues in glycoproteins. Sialyl Lewis $\mathrm{X}$ and other fucosylated selectin ligands that are required in the rolling phase of adhesion cannot be synthesized in LAD II patients, leading to a defect in leukocyte rolling (Fig. 1), while firm adhesion is partially conserved [54-56]. Similar to LAD I patients, subjects with LAD II also present neutrophilia and gingivitis, but they also develop severe psychomotor and growth retardation [57]. Because of the general defect of fucose incorporation in the $\mathrm{H}$ antigen, LAD II patients present the Bombay blood group [54].

LAD III, originally described as LAD I variant, is an autosomal recessive disorder caused by mutations of FERMT3 gene that encodes for kindlin-3, a protein expressed by hematopoietic cells that is required for integrin activation that affects both leukocytes and platelets [58, 59]. Despite normal expression of beta2 integrin, leukocytes from LAD III patients display abnormal adhesion and migration in response to chemoattractants leading to an immune defect similar to LAD I (Fig. 2) [60,61]. Patients with LAD III present with neutrophilia and increased susceptibility to bacterial infections as observed in LAD I patients, but they also present bleeding tendency. This symptom is related to platelet defects of LAD III patients. In platelets of patients with kindlin-3 deficiency, beta 3 integrin activation is also impaired, resulting in abnormal platelet aggregation and abnormal function. Impaired functioning of integrins in these patients also affects osteoclast functions in bone resorption and can occasionally result in manifestations resembling osteoporosis $[62,63]$. 


\section{Concluding remarks}

As highlighted in this Viewpoint with selected examples, leukocyte trafficking can be altered at different levels in primary immunodeficiencies. Leukocyte adhesion deficiencies (including LAD I, II, and III) are characterized by a general defect in leukocyte rolling, adhesion, and/or transendothelial migration leading to leukocytosis and to poor inflammatory responses against microbial infections of the skin. In contrast, in patients with truncating mutations of CXCR4, abnormal functioning of this chemokine receptor leads to reduced release of mature neutrophils from bone marrow and to neutropenia with myelokathexis (neutrophil retention in the bone marrow). Finally, patients with disorders related to the Wiskott-Aldrich syndrome protein present a broad array of immunologic defects that affect innate and adaptive immunity as a consequence of defects in actin polymerization, a step crucial for chemotactic responses.

Understanding the molecular basis of the primary immunodeficiencies that are characterized by abnormal trafficking will have an impact on the identification of novel therapeutic strategies for these rare diseases and might provide new pharmaceutical tools for the treatment of more common immune-mediated disorders, including autoimmune diseases and allergy.

Acknowledgements: This work was funded by Telethon Italia GGP10170A, eRare, Fondazione Cariplo, and PRIN 2009 to R.B.

Conflict of interest: The author declares no financial or commercial conflict of interest.

\section{References}

1 Hernandez, P. A. et al., Nat. Genet. 2003. 34: 70-74.

2 Gulino, A. V. et al., Blood 2004. 104: 444-452.

3 Balabanian, K. et al., Blood 2004. 105: 2449 2457.

4 Dotta, L. et al., Curr. Mol. Med. 2011. 11: $317-$ 325.

5 Beaussant Cohen, S. et al., Orphanet J. Rare Dis. 2012. 7: 71-85.

6 Liu, Q. et al., Blood 2012. 120: 181-189.

7 Haribabu, B. et al., J. Biol. Chem. 1997. 272 28726-28731.

8 Tassone, L. et al., J. Allergy Clin. Immunol. 2009. 123: 1170-1173.
9 Kawai, T. et al., Blood 2007. 109: 78-84.

10 Balabanian, K. et al., Blood 2012. 119: 57225730.

11 Badolato, R. et al., J. Pediatr. 2012. 161: 763-765.

12 Zou, Y. R. et al., Nature 1998. 393: 595-599.

13 Sierro, F. et al., Disrupted cardiac development but normal hematopoiesis in mice deficient in the second CXCL12/SDF-1 receptor, CXCR7. Proc. Natl. Acad. Sci. USA 2007. 104: 14759-14764.

14 Kawai, T. et al., Exp. Hematol. 2005. 33: 460468.

15 Walters, K. B. et al., Blood 2010. 116: 28032811.

16 McDermott, D. H. L. et al., Blood 2011. 118: 4957-4962.

17 Mc Guire, P. J. et al., Clin. Immunol. 2010. 135: 412-421.

18 Mentzer, W. C. Jr. et al., Br. J. Haematol. 1977. 36: 313-322.

19 Tassone, L. et al., Blood 2010. 116: 4870-4873.

20 Dale, D. C. et al., Blood 2011. 118: 4963-4966.

21 Thrasher, A. J. and Burns, S. O., Nat. Rev. Immunol. 2010. 10: 182-192.

22 Antón, I. M. et al., Trends Cell Biol. 2007. 17: 555-562.

23 Ochs, H. D., Immunol. Res. 2009. 44: 84-88.

24 Jin, Y. et al., Blood 2004. 104: 4010-4019.

25 Notarangelo, L. D. et al., Curr. Opin. Hematol. 2008. 15: $30-36$

26 Lanzi, G. et al., J. Exp. Med. 2012. 209: 29-34.

27 Devriendt, K. et al., Nat. Genet. 2001. 27: 313317

28 Ancliff, P. J. et al., Blood 2006. 108: 21822189.

29 Moulding, D. A. et al., J. Exp. Med. 2007. 204: 2213-2224.

30 Badolato R. et al., J. Immunol. 1998. 161: 10261033.

31 Zicha, D. et al., Br. J. Haematol. 1998. 101: 659665.

32 Burns, S. et al., Blood 2001. 98: 1142-1149.

33 Allavena, P. et al., Eur. J. Immunol. 2001. 31: 3413-3421.

34 Ishihara, D. et al., PLoS One 2012. 7: e30033.

35 Bouma, G. et al., Blood 2007. 110: 4278-4284.

36 Lang, P. A. et al., J. Allergy Clin. Immunol. 2013. 131: 815-824

37 Bouma, G. et al., Blood 2011. 118: 2492-2501.

38 Snapper, S. B. et al., J. Leukoc. Biol. 2005. 77: 993-998.

39 Sanz, M. J. and Kubes, P., Eur. J. Immunol. 2012. 42: 278-283.

40 Kumar, S. et al., Blood 2012. 120: 3563-3574.

41 Haddad, E. et al., Blood 2001. 97: 33-38.

42 Westerberg, L. S. et al., Blood 2008. 112: 41394147

43 Recher, M. et al., Blood 2012. 119: 2819-2828.

44 Westerberg, L. S. et al., Blood 2012. 119: 3966 3974.

45 Lacout, C. et al., Blood 2003. 102: 1282-1289.

46 Crowley, C. A. et al., N. Engl. J. Med. 1980. 302: 1163-1168.
47 van de Vijver, E., et al., Blood Cells Mol. Dis 2012. 48: 53-61.

48 Hanna, S. and Etzioni, A., Ann. NY Acad. Sci. 2012. 1250: 50-55.

49 Luo, B. H. et al., Annu. Rev. Immunol. 2007. 25 619-647.

50 Fiorini, M. et al., J. Leukoc. Biol. 2002. 72: 650656

51 Qasim, W. et al., Pediatrics 2009. 123: 836-840.

52 Lubke, T. et al., Nat. Genet. 2001. 28: 73-76.

53 Luhn, K. et al., Nat. Genet. 2001. 28: 69-72.

54 Etzioni, A. et al., Immunodeficiency 1993. 4: $307-$ 308

55 Hidalgo, A. et al., Blood 2003. 101 1705-1712.

56 Helmus, Y. et al., Blood 2006. 107: 3959-3966.

57 Gazit, Y., et al., J. Clin. Immunol. 2010. 30: 308313.

58 Etzioni, A. and Alon, R., Blood 2007. 110: 41294130.

59 Kuijpers, T. W. et al., Blood 2009. 113: 47404746.

60 Mory, A. et al., Blood 2008. 112: 2591.

61 Kuijpers, T. W. et al., J. Clin. Invest. 1997. 100: 1725-1733.

62 Kilic, S. S. and Etzioni, A., J. Clin. Immunol. 2009. 29: 117-122.

63 Schmidt, S. et al., J. Cell Biol. 2011. 192: 883897.

Correspondence: Prof. Raffaele Badolato, Istituto di Medicina Molecolare "Angelo Nocivelli”, Universita' di Brescia, c/o Spedali Civili, 25123 Brescia, Italy

Fax: +39-0303388099

e-mail: badolato@med.unibs.it

Received: 15/11/2012

Revised: 7/4/2013

Accepted: 24/4/2013

Accepted article online: 13/5/2013

Keywords: CXCR4 - Leukocyte adhesion • Primary immunodeficiency • Wiskott-Aldrich • WHIM

Abbreviations: CT: carboxy-terminal . GBD: GTPase-binding domain . HPV: human papilloma virus . LAD: leukocyte adhesion deficiency . pDC: plasmacytoid dendritic cell WASP: Wiskott-Aldrich syndrome protein . WH1: WASP homology 1 . WHIM: warts, hypogammaglobulinemia, infections, and myelokathexis - WIP: WASP interacting protein - XLN: X-linked neutropenia - XLT: Xlinked thrombocytopenia

See accompanying Viewpoint: http://dx.doi.org/10.1002/eji.201243183

The complete Cells in Motion Viewpoint series is available at: http://onlinelibrary.wiley.com/doi/10. 1002/eji.v43.6/issuetoc 